\title{
The EMC Effect in $A=3$ Nuclei
}

A Dissertation Presented for the Doctor of Philosophy

Degree

The University of Tennessee, Knoxville

Jason Earl Bane

December 2019 
(C) by Jason Earl Bane, 2019

All Rights Reserved. 


\section{Acknowledgments}

I would like to thank everybody that played a role in completing this step of my life. I know there is no way that I could go one by one through every person that helped and thank them. I am sure that it would take too many pages and then I would leave somebody out. There are a few special people, that I would like to call out.

First, I would like to thank my brother, James for the courage to change career paths and my parents, Barb, Howard, and Deb for the support and guidance. Thanks to Dallas and Jennifer, for housing me for a few months. Thank you, Dr. Fomin, for targeting my weakness, the beach, and giving me the chance to join the JLab community. Also, I would like to thank Dr. Fomin for supporting my research and guiding me through the swamps of PhD. research. I would like to thank the JLab community especially Dr. Higinbotham for accepting me into the community and helping through this journey including keeping the office door open for me to drop in a million times. I would also like to thank my committee for guiding me through my defense and allowing me to graduate.

I would really like to thank the extended tritium family of grad students and postdocs. Working with such a great group of people from around the world has truly been amazing! Thank you, Sheren, Scott, Jessica, Tyler H., Hanjie, Dien, Mike, Tong, Shujie, Rey,

Johnathan, Tyler K., Nathaly, Bishnu, Evan, Florian, Luke, Marco, and Zhihong. Lastly and I think the person deserving of the most thanks, is my wife, Callie. Thank you so much for everything! Specially thank you for dropping everything and coming on this adventure with me. I would like to thank the DOE and JSA for financial support throughout my time at JLab. 


\section{Abstract}

The European Muon Collaboration(EMC) discovered an unexpected and puzzling result in 1983 when comparing the deep inelastic scattering(DIS) nuclear structure functions of Deuterium and Iron. The per-nucleon structure functions were found to be different for the two nuclei, rather than a simple average over the proton and neutron structure functions. In subsequent experiments, this phenomenon was confirmed for additional nuclei, with the magnitude approximately scaling with the density. The exact mechanism leading to this inmedium structure function modification has not been identified. I will discuss recent results from JLab exploring the EMC effect by using an electron beam to probe two mirror nuclei, Helium-3 and Tritium. 


\section{Table of Contents}

1 Introduction $\quad 1$

1.1 Electron scattering . . . . . . . . . . . . . . . . . . . 2

1.2 Deep inelastic scattering . . . . . . . . . . . . . . . 7

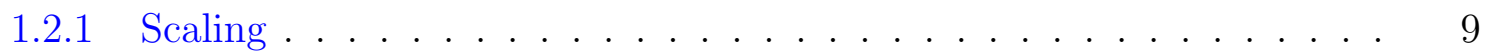

1.2.2 Quark Parton Model ........................ 9

2 EMC Effect $\quad 14$

2.1 European Muon Collaboration . . . . . . . . . . . . . . . 14

2.2 Ratios of Cross Sections and Structure Functions . . . . . . . . . . . . 15

2.3 EMC Experiments . . . . . . . . . . . . . . . . . . . 17

2.3.1 Experiments at CERN . . . . . . . . . . . . . . . 17

2.3.2 Experiments at SLAC . . . . . . . . . . . . 18

2.3.3 HERMES at DESY . . . . . . . . . . . . . . . . 20

2.3.4 Experiments at Jefferson Lab . . . . . . . . . . . . . . . . . 20

2.4 EMC Theory and Models . . . . . . . . . . . . . . . . . . 21

2.4.1 Multiquark Cluster . . . . . . . . . . . . . . 23

2.4.2 Nuclear Binding . . . . . . . . . . . . . . . . . . 23

2.4.3 Medium Modification . . . . . . . . . . . . . . . . . . 24

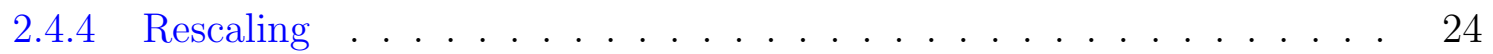

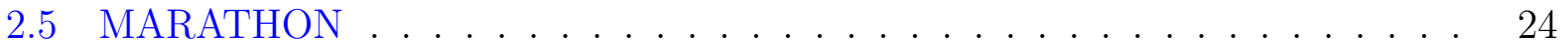

3 Experimental Setup $\quad 26$

3.1 Thomas Jefferson Lab . . . . . . . . . . . . . . . . . . . . . . 26 


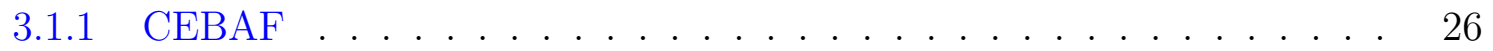

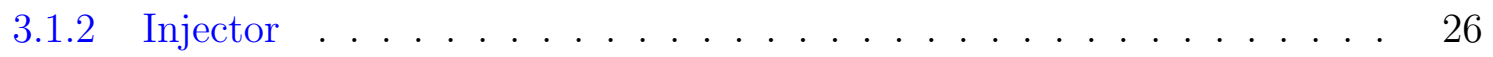

3.1 .3 Accelerator . . . . . . . . . . . . . . . . . . 27

3.2 Hall A Beam Line . . . . . . . . . . . . . . . . . . . . . . . . . . . . . . . 29

3.2 .1 Beam Position Monitors . . . . . . . . . . . . . . . . . . 30

3.2 .2 Raster . . . . . . . . . . . . . . . . . . . . . . . . . 33

3.2 .3 Beam Energy . . . . . . . . . . . . . . . . . . . . . . . 35

3.2 .4 Beam Current Monitors . . . . . . . . . . . . . . . . . . . . . . 37

3.3 Target . . . . . . . . . . . . . . . . . . . . . . . . . . . . 39

3.4 High Resolution Spectrometers ．.. . . . . . . . . . . . . . . . . 40

3.4.1 Vertical Drift Chambers . . . . . . . . . . . . . . . . . . 42

3.4 .2 Scintillators . . . . . . . . . . . . . . . . . . . . 45

3.4 .3 Cherenkov . . . . . . . . . . . . . . . . . . . . . . . . 45

3.4 .4 Calorimeter . . . . . . . . . . . . . . . . . . . 47

3.5 Trigger Setup . . . . . . . . . . . . . . . . . . . . . . . . 49

3.6 Kinematic Settings . . . . . . . . . . . . . . . . . . . 50

4 Data Analysis $\quad 53$

4.1 Tracking . . . . . . . . . . . . . . . . . . . . . 54

4.2 Electron Selection . . . . . . . . . . . . . . . . . . . . . . 58

4.2 .1 Acceptance Cuts . . . . . . . . . . . . . . . . . . . . . . 59

4.2 .2 Identification Cuts . . . . . . . . . . . . . . . . . . 60

4.3 Efficiencies. . . . . . . . . . . . . . . . . . . . . 62

4.3.1 Computer and electronic Livetime . . . . . . . . . . . . . . 62

4.3 .2 Particle Identification Efficiency . . . . . . . . . . . . . . . 63

4.3 .3 Trigger Efficiency . . . . . . . . . . . . . . . . . . . . . 65

4.3 .4 Tracking Efficiency . . . . . . . . . . . . . . . . 66

4.4 Background Subtraction _. . . . . . . . . . . . . . . 67

4.4 .1 End Caps . . . . . . . . . . . . . . . . . . . . . 68

4.4 .2 Pair Produced Electrons _ . . . . . . . . . . . . . . . . . . 69 
4.4.3 Beta Decay of ${ }^{3} \mathrm{H} \ldots \ldots \ldots \ldots$. . . . . . . . . . . . . 70

4.5 Luminosity . . . . . . . . . . . . . . . . . . . . 72

4.6 Yield . . . . . . . . . . . . . . . . . . . . . . . . . 74

4.7 Acceptance . . . . . . . . . . . . . . . . . . 75

4.8 Monte Carlo Ratio Method . . . . . . . . . . . . . . . . . . . . . . 75

4.8.1 Monte Carlo Simulation _. . . . . . . . . . . . . . . 76

4.8.2 Monte Carlo Comparison . . . . . . . . . . . . . . . . . . . . . . 81

5 Results $\quad 83$

5.1 DIS Cross Section . . . . . . . . . . . . . . . . . . . . . . . . 83

5.2 Cross Section Error Analysis . . . . . . . . . . . . . . . . . . . 84

$5.2 .1 \quad$ Normalized Yield Error . . . . . . . . . . . . . . . . . . . . . 85

5.2 .2 Monte Carlo Yield Error . . . . . . . . . . . . . . . . . . . . 87

5.2 .3 Cross Section Model Error . . . . . . . . . . . . . . . . . . . . 88

5.3 EMC Ratios . . . . . . . . . . . . . . . . . . . . . . . . . . . . . . . . 89

5.3 .1 Isoscalar Correction . . . . . . . . . . . . . . . . . . . . 89

5.4 EMC Effect . . . . . . . . . . . . . . . . . . . . . . 91

5.4 .1 Ratio of EMC Effects . . . . . . . . . . . . . . . . . 91

6 Conclusion $\quad 95$

$\begin{array}{ll}\text { Bibliography } & 97\end{array}$

$\begin{array}{lr}\text { Appendices } & 106\end{array}$

A Cross Section Tables . . . . . . . . . . . . . . . . . 107

B EMC Simulation . . . . . . . . . . . . . . . . . . . 111

B.1 Investigation $\ldots \ldots \ldots \ldots \ldots \ldots \ldots \ldots \ldots \ldots \ldots$

B.2 Transformation . . . . . . . . . . . . . . . . . . . . . 112

B.3 Results . . . . . . . . . . . . . . . . . . . . . . . . . . 115

$\begin{array}{lr}\text { Vita } & 116\end{array}$ 


\section{List of Tables}

3.1 Kinematics originally planned for the MARATHON experiment inlcuding an estimation of time required for three of the gas targets in hours. Estimations provided by John Arrington and Zhihong Ye[56]. . . . . . . . . . . . . . . . 52

3.2 Kinematic settings used during the MARATHON experiment. Kinematic 115 for LHRS, and kinematic 16 using RHRS. The good electron count is in units of thousands. . . . . . . . . . . . . . . . . .

4.1 Livetime for each kinematic setting during the MARATHON experiment calculated using trigger $2 . \ldots \ldots$. . . . . . . . . . . . . . 63

4.2 Table of gas target density thickness and uncertainty [66]. . . . . . . . . 73

4.3 Table of density correction parameters [78] . . . . . . . . . . . . . 74

5.1 Relative Error Contributions for Cross Section for a selection of bins. This table contains the summary of relative error to the cross section for the yield, Monte Carlo, and Cross Section Model. Then summed in quadrature is the total relative error for the cross section. . . . . . . . . . . . . . .

5.2 This table provides a summary of the error analysis for the Corrected Normalized Electron Yield. Containing the relative error for the bin statistics, background subtraction, efficiencies, and luminosity calculation. Including the total error on the Yield analysis for a selection of bins over the entire range of kinematics. . . . . . . . . . . . . . . . . . .

5.3 This table contains a summary of the relative error for the Monte Carlo yield extraction and the model cross section. Also, I include the errors add in quadrature for these two analysis parts. . . . . . . . . . . . 
A.1 Cross section table for ${ }^{3} \mathrm{H}$. . . . . . . . . . . . . . . . 108

A.2 Cross section table for ${ }^{3} \mathrm{H} . \ldots \ldots \ldots$. . . . . . . . . . . . 109

A.3 Cross section table for D. . . . . . . . . . . . . . . . . . . 110 


\section{List of Figures}

1.1 Simple Feynman diagram of an electron scattering from a proton [40]. . . . . 3

1.2 The ratio of the experimentally measured cross section to the Mott cross section verses $\tan ^{2} \theta / 2$ for a $Q^{2}$ of $2.5 \mathrm{GeV}^{2} / \mathrm{c}^{2}$. [1] . . . . . . . . . .

1.3 Electron-proton scattering for incident energy of $4.9 \mathrm{GeV}$ and scattering angle of $10^{\circ}[19] \ldots \ldots \ldots \ldots \ldots \ldots$

1.4 Measurements of the proton structure function $F_{2}\left(x, Q^{2}\right)$ for different $x$ settings[38]. The $Q^{2}$ independence shows that there is nothing smaller than

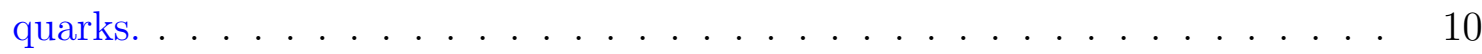

1.5 Data from SLAC, showing the ratio of the structure functions $2 x \cdot F_{1}(x)$ and $F_{2}(x)$ vs. $x[76,75] \ldots \ldots \ldots \ldots$

1.6 Structure function results measured from three lepton-nucleon scattering experiments $[16] . \ldots \ldots \ldots \ldots$

1.7 Elementary particles including leptons, quarks, and bosons with mass, charge,

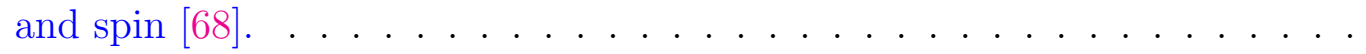

2.1 Graph of the ratio of A/D structure functions vs $x$ from the EMC. [52, 12]. . 15

2.2 EMC effect from the BCDMS collaboration [18] . . . . . . . . . . . . 17

2.3 EMC ratios from SLAC. The plot shows the $Q^{2}$ average cross section ratios with isoscalar corrections for different nuclei [45]. . . . . . . . . . . . . 19

2.4 The dependence of the atomic mass number on the EMC effect[45]. . . . . 20

2.5 Ratio of nuclear to D per nucleon cross sections corrected for neutron excess[9]. The JLab data in red is compared with SLAC data in Blue [45] and BCDMS data in green [18]. The red lines are model extractions from [48] . . . . . . . 
2.6 Isoscalar EMC effect as function of nuclear density $[79] \ldots \ldots \ldots \ldots \ldots$

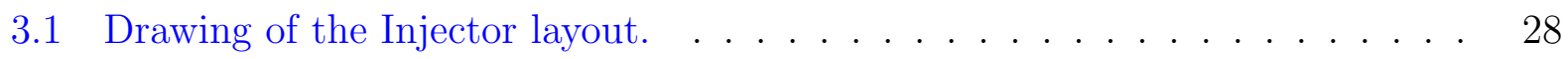

3.2 Schematic Layout of CEBAF. . . . . . . . . . . . . . . . . . 29

3.3 A schematic layout of the beam line in Hall. [4] . . . . . . . . . . . . 30

3.4 BPM design diagram, from JLab instrumentation group. Beam direction is from left to right $[86] . \ldots \ldots \ldots \ldots \ldots \ldots$

3.5 BPM design diagram, looking down the beam line[86]. The diameter of the beam line is restricted to $63.5 \mathrm{~mm} \mathrm{[4].} \mathrm{\ldots} \mathrm{\ldots} \mathrm{.} \mathrm{.} \mathrm{.} \mathrm{.} \mathrm{.} \mathrm{.} \mathrm{.} \mathrm{.} 32$

3.6 A schematic layout of a harp fork $[86] \ldots \ldots 33$

3.7 The $\mathrm{X}$ and $\mathrm{Y}$ position comparison for harp to BPM for a bulls eye scan before BPM calibration. . . . . . . . . . . . . . . . . 34

3.8 The X and Y position comparison for harp to BPM for a bulls eye scan after BPM calibration. . . . . . . . . . . . . . . . 34

3.9 The $\mathrm{X}$ and $\mathrm{Y}$ current of the raster with a carbon hole. The size of the carbon hole is fit with a radial sigmoid[49]. . . . . . . . . . . . . . 36

3.10 Hall A Current Monitor components [35] . . . . . . . . . . . . . . . 37

3.11 BCM calibration, changing the current, observing the rate for dnew, a

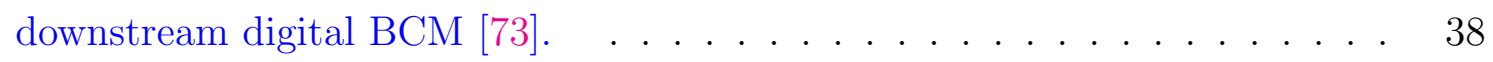

3.12 The relationship between unser current and BCM frequency for BCM calibration $[73] . \ldots \ldots \ldots \ldots$

3.13 Target Images . . . . . . . . . . . . . . . . . . . . . . . . . . 40

3.14 A side view of a HRS $[4] . \ldots \ldots \ldots \ldots \ldots \ldots$

3.15 A view of both the left (top) and right (bottom) detector stacks inside the left and right HRS [4]. . . . . . . . . . . . . . . . . . . . . 42

3.16 A sketch of the two VDC planes in the HRSs with a particle traveling through the detector at $45^{\circ} \cdot[39] . \ldots \ldots \ldots \ldots \ldots \ldots \ldots$

3.17 Histograms of VDC signals before (left) and after (right) calibration of t0[17]. 44

3.18 Top down depiction inside the GC $[15] \ldots \ldots$. . . . . . . . . . . . 46 
3.19 The raw signal captured from a single Cherenkov PMT, PMT[1], with a fit to the pedestal peak and a line draw to demonstrate its ADC channel number.

3.20 Left: The sum of all ADC channels from the LHRS calorimeters. Right: The total energy deposited into the LHRS calorimeter scaled by the momentum setting. Electron cuts have been applied. . . . . . . . . . . . .

3.21 Scematic drawing of the trigger logic and timing for the MARATHON experiment $[51] \ldots \ldots \ldots \ldots \ldots \ldots$

3.22 A kinematic coverage plot, demonstrating the $Q^{2}$ coverage for $x$ and Theta. Also the relationship between $x$ and Theta. The band around the points represents the approximate spectrometer acceptance in the y axis. . . . . .

4.1 The TCS for an electron scattering event as seen from above. The event happens at $z_{\text {react }}$ distance from the Hall center. $\mathbf{L}$ is the distance from the Hall center to the sieve plane. $\mathbf{D}$ is the horizontal displacement of the spectrometer axis from the ideal position. $\Theta$ is the spectrometer's central angle [62]. . . .

4.2 Simulation of events to demonstrate the acceptance of the spectrometer. Black events have been generated with no acceptance effects, Red points include a cut to only allow events that traverse the entire spectrometer. The Blue lines are example locations of cuts used in event selection to exclude the edge of

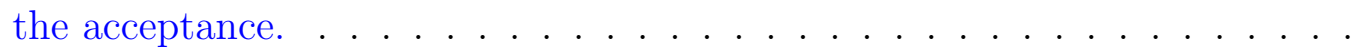

4.3 A scan of the PID efficiency for a cut in each layer of the calorimeters. . . .

4.4 Two dimensional plot of the Cherenkov sum versus Total Energy deposited, including electron sampling in teal and non-electron sampling in red. . . . .

4.5 Electrons and other background particles identified via cuts in the total calorimeter and the gas Cherenkov shown in the individual layers of the calorimeters (top) and the Cherenkov (bottom). Sampling cuts for Electrons in teal and Non-Electrons in red. . . . . . . . . . . . . . . . . . .

4.6 The PID efficiency for the Cherenkov and both layers of the calorimeter,including the overall total PID efficiency for each of the gas targets at all of the kinematics. . . . . . . . . . . . . . . . . . . . 
4.7 Trigger efficiency of trigger 2 for different targets at all kinematics calculated

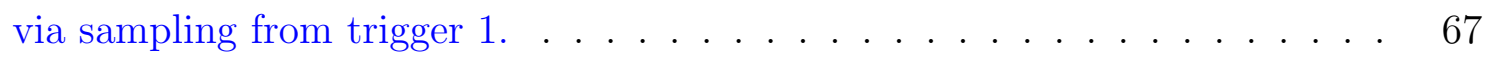

4.8 Tracking efficiency of the VDCs for different targets at all kinematics. . . . 68

4.9 Comparison of the scattering vertex along the $\mathrm{z}$ axis for the empty target (EM) and the gas targets at kin. 4. . . . . . . . . . . . . . 69

4.10 The ratio of positron events to electrons for ${ }^{3} \mathrm{H}[82] . \quad$. . . . . . . . . 70

4.11 The amount of ${ }^{3} \mathrm{He}$ in the ${ }^{3} \mathrm{H}$ cell in reference to the total amount of material in the cell as a function of time. Included are bands of time for different sections of the ${ }^{3} \mathrm{H}$ run group's plan [60]. . . . . . . . . . . . . . . . . 71

4.12 Luminosity normalized corrected yield for ${ }^{2} \mathrm{D}$. The yield for each kinematic is shown as a different color and marker, with the combined yield as a gray ' $\mathrm{x}$ '. $\quad 75$

4.13 Model cross section for ${ }^{3} \mathrm{H}$ at kinematic 1 for scattered angle and momentum. 77

4.14 Lowest order Feynman diagrams for inclusive lepton-nucleon scattering [33]. $\quad 78$

4.15 Radiative correction for ${ }^{3} \mathrm{H}$ at kinematic 1, for scattered angle and momentum. 79

4.16 Monte Carlo to Data comparison for target plane variables, top left $\delta$,bottom left y, top right $\theta$, bottom right $\phi$. Run 1207, kinematic 1 on carbon foil. . . 81

4.17 Monte Carlo to Data comparison for focal plane variables, top left $\theta$, bottom left $\phi$, top right $x$, bottom right $y$. Run 1207, kinematic 1 on carbon foil. $\quad . \quad 82$

4.18 The ratio of data to Monte Carlo yield in bins of $x$ for three gas targets. . . 82

5.1 Experimentally measured cross section using the Monte Carlo ratio method for ${ }^{3} \mathrm{H},{ }^{3} \mathrm{He}$, and D. . . . . . . . . . . . . . . . . . . 84

5.2 The A/D ratio for ${ }^{3} \mathrm{He}$ and ${ }^{3} \mathrm{H}$ from my analysis. Also included, EMC analysis from E03103[79] and the EMC ratios from a DIS scattering model from Arie Bodek model [23]. . . . . . . . . . . . . . . . . . . . . . 90

5.3 Isoscalar correction factor for the ${ }^{3} \mathrm{H}$ EMC ratio from four different models discussed in this section. . . . . . . . . . . . . . . . . . 92

5.4 Isoscalar correction factor for the ${ }^{3} \mathrm{He}$ EMC ratio from four different models discussed in this section. . . . . . . . . . . . . . . . . . 92 
5.5 Isoscalar corrected EMC ratio for ${ }^{3} \mathrm{He}$. The point to point error bars are the total systemic and statical errors for the EMC effect. . . . . . . . . . . .

5.6 Isoscalar corrected EMC ratio for ${ }^{3} \mathrm{H}$. The point to point error bars are the total systemic and statical errors for the EMC effect. . . . . . . . . . . . .

5.7 Ratio of the isoscalar corrected ${ }^{3} \mathrm{H}$ EMC Effect and ${ }^{3} \mathrm{He}$ EMC Effect. The error bar is statistical error only, while the band includes statical and systematic

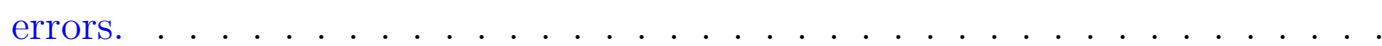

B.1 Example of the electron beam (red) with an energy of $2.5 \mathrm{GeV}$ and the proton (blue) with angle of $45^{\circ}$ in respect to the electron and with a momentum of $0.5 \mathrm{GeV} /$ c. . . . . . . . . . . . . . . . . . . .

B.2 Vector representations of the momentum for the incoming electron (red) and target proton (blue) with units of $\mathrm{GeV}$ for each phase of their transformations before scattering. . . . . . . . . . . . . . . . . . . . . . . . . . 113

B.3 Vector representations of the momentum for the incoming electron (red) and target proton (blue) with units of $\mathrm{GeV}$ for each phase of their transformations after scattering). . . . . . . . . . . . . . . . . . . . . . 114

B.4 Simulation results for fixed momentum protons. Three runs with unique proton momentum. . . . . . . . . . . . . . . . . . . . . 115 


\section{Chapter 1}

\section{Introduction}

Understanding the world around us is the goal of every scientist, from the chemist that experiments with the formation of atoms to the geologist exploring the process of rock formations. Nuclear physicists focus on studying the fundamental constituents of matter, the building blocks of nature. Physicists use scattering experiments at accelerator facilities, like CERN in Switzerland, DESY in Germany, BATES in Massachusetts, Thomas Jefferson National Accelerator Facility in Virginia, and many others, to study the protons and neutrons that make up a nucleus and the constituents that form the internal structure of a nucleon. These experiments allow physicists to probe inside a nucleus to observe the internal structure and to investigate the interactions between the quarks and gluons. Many of the experiments are designed to confirm existing results while also expanding on unique ideas.

In the last century, there have been numerous breakthroughs in the fields of nuclear and particle physics. Rutherford discovered the proton by bombarding light nuclei with alpha particles to produce the reaction,

$$
{ }^{14} \mathrm{~N}+{ }^{4} \mathrm{He} \rightarrow{ }^{17} \mathrm{O}+\mathrm{p}
$$

This reaction allowed Rutherford to conclude that the Hydrogen nucleus was an elementary constituent of atomic nuclei [76]. In the late 1950s, experimental results published by W. McAllister and R. Hofstadter exposed some of the internal structure of the proton [40, 65]. 
The European Muon Collaboration (EMC) produced results in the early 1980s showing a difference between the internal structure of the D nucleus and Fe [52, 79]. In the current era, scientific labs can produce beams of leptons, hadrons, and heavy ions. These beams can be produced with a large energy spread from "cold" neutrons of $10^{-2} \mathrm{eV}$ to protons of $10^{12} \mathrm{eV}$ [76]. The data received from scattering experiments using beams with a complex structure like alpha particles of heavy ions contain information about the target, the beam, and the interaction between the two.

This thesis will discuss using deep inelastic scattering to study the internal structure of two light nuclei and gain a better understanding of the effects of the slight difference between these two light nuclei as part of the E12-010-103 experiment. The discussion will include the motivations, approach, and the outcome from one analysis technique.

\section{$1.1 \quad$ Electron scattering}

Deciphering and analyzing data from scattering experiments that use complex beams can be convoluted because the scattering interaction contains information about the internal structure of the target and the beam along with the complex interactions and forces between the two [76]. In order to remove some of the complexity in scattering experiments, one may employ highly relativistic electrons. Electrons being point-like particles without any internal structure allow the elimination of some of the analysis difficulties due to the complex nature of the internal structure of more complex scattering tools. The interaction between an Electrons and the target either a nucleus, nucleon, or quark interact via the exchange of a virtual photon. Using quantum electrodynamics (QED), these interactions can accurately be described by the well known electromagnetic interaction, using single photon exchange $[76]$.

The electromagnetic interaction describes the coupling of fundamental particles via their electric charge. The interaction between two electrically charged particles begins when a virtual photon is emitted. The amplitude for the emission of a photon is proportional to 


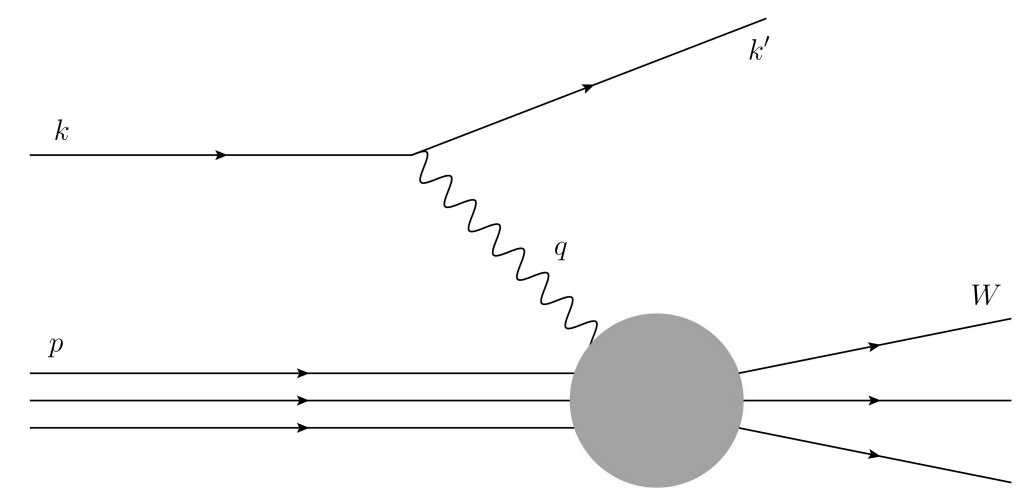

Figure 1.1: Simple Feynman diagram of an electron scattering from a proton [40].

$\sqrt{\alpha}$, where $\alpha$ is the fine structure constant. Higher order terms of this process contribute very little due to the coupling constant $\alpha \approx 1 / 137$, being much smaller than one [76].

The Feynman diagram in figure 1.1 represents an electron scattering from a proton. The incoming or incident electron's four-momentum is described as $\mathrm{k}=(E, \vec{k})$, and the scattering electron's four-momentum is represented by $k^{\prime}=\left(E^{\prime}, \vec{k}^{\prime}\right)$. The exchange of the virtual photon in this electromagnetic interaction is defined by the four-momentum transfer $q . Q^{2}$ the square of the momentum transfer is the mass of the virtual photon that provides the interaction between the lepton and hadron [68].

$$
Q^{2} \equiv-q^{2}=4 E E^{\prime} \sin ^{2}(\theta / 2)
$$

In equation $1.2, E$ is the electrons incident energy and $E^{\prime}$ is the energy of the scattered electron. $\Theta$ is the angle that describes the deflection of the $E^{\prime}$ vector from the electron's incident path. Along with $Q^{2}$, the variables $\nu, W$, and $x_{B}$ are used to narrate the evolution of the electron scattering process. $\nu$, defined as $P \cdot q / M$. Where $P$ is the 4 -vector of the target proton. In the laboratory frame, $\nu$ can be described by equation 1.3. The transformation to the laboratory frame allows the use of the resting nature of the target proton. Therefore $P=(M c, 0)$ and $q=\left(\left(E-E^{\prime}\right) / c, \boldsymbol{q}\right)$.

$$
\nu=E-E^{\prime}
$$


Simply, $\nu$ is the magnitude of energy loss by the electron during the scattering interaction. The invariant mass of the system, W, defines the hadronic state produced by the scattering event.

$$
W^{2} \equiv(q+p)^{2}=M^{2}+2 M \nu-Q^{2}
$$

In the general case of electron scattering off of a free proton or neutron elastically, the scattered energy of the electron will be a function of the incident electron's energy and the scattered angle of the electron, shown in the following equation.

$$
E^{\prime}=\frac{E}{1+\frac{E}{M c^{2}}(1-\cos \theta)}
$$

A scattering event with the invariant mass equal to the mass of the nucleon, $M$, falls in the regime of elastic scattering and the final state of hadron is a recoiling proton. Increasing the $W$ above $M$ will transform the scattering interaction from an elastic scattering interaction to an inelastic scattering event due to the excited state of the scattered byproduct.

The intrinsic likelihood of an event with a certain $Q^{2}, \nu$, and $W$ is defined by the scattering cross section. An electron scattering off of a target with a charge of $Z \times e$ can be described by the Rutherford cross section. Povh et. al. [76] details the Rutherford cross section as:

$$
\left(\frac{d \sigma}{d \Omega}\right)_{\text {Rutherford }}=\frac{\left(z Z e^{2}\right)^{2}}{\left(4 \pi \epsilon_{0}\right)^{2} *\left(e E_{\text {kin }}\right)^{2} \sin ^{4}(\theta / 2)} .
$$

In the early 1920s, German physicists Stern and Gerlach performed an experiment with a beam of silver atoms. The SternGerlach experiment measured the deflection of a beam of silver atoms from an inhomogeneous magnetic field[47]. The observations made by Stern and Gerlach demonstrated that particles bear an intrinsic angular momentum. In 1925, a forbidden spectral line of ionized He raised questions of the current understanding of the quantum numbers used. This forbidden line lead to the discovery of electron spin by Uhlenbeck and Gloudsmit[46]. The Mott cross section is the evolved version of the Rutherford cross section. The Rutherford cross section neglects the spin of an electron and the target. Evolving the Rutherford cross section allows for the modifications needed to 


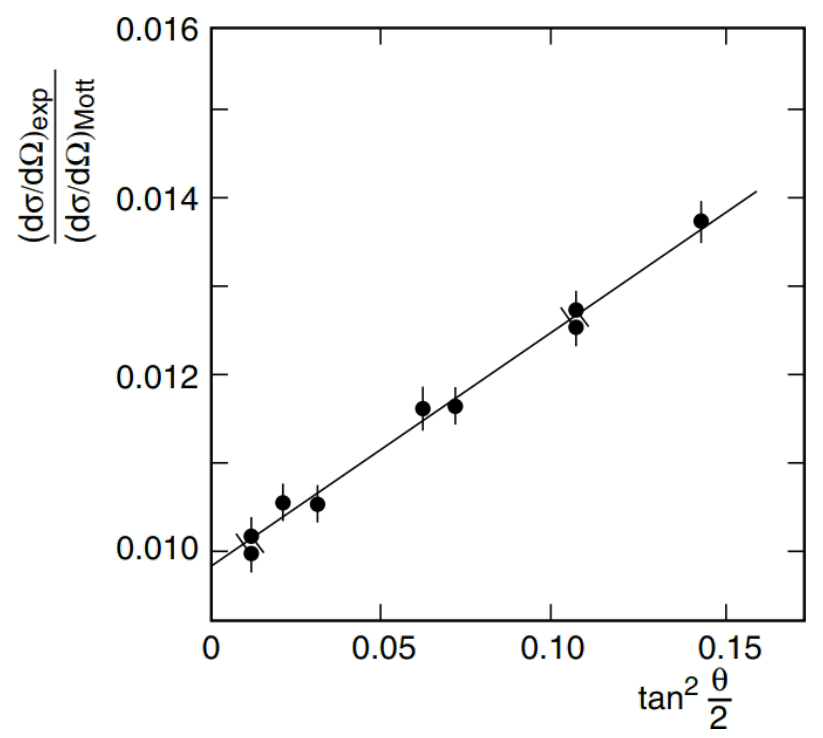

Figure 1.2: The ratio of the experimentally measured cross section to the Mott cross section verses $\tan ^{2} \theta / 2$ for a $Q^{2}$ of $2.5 \mathrm{GeV}^{2} / \mathrm{c}^{2}$. [1]

include the intrinsic spin of the target and electron. The Mott cross section is described in equation $1.7[54,76]$. Where $\alpha$ is the fine structure constant. This constant is related to the strength of the interaction between an electron and proton[68].

$$
\left(\frac{d \sigma}{d \Omega}\right)_{M o t t}=\frac{4 Z^{2} \alpha^{2}(\hbar c)^{2} E^{\prime 2}}{|\boldsymbol{q} c|^{4}} \cos ^{2}(\theta / 2)
$$

The modification to the Mott scattering cross section equation to include a spin degree of freedom is shown in equation 1.8. $\tau$ is used in the cross section formalism to account for the magnetic moment of a nucleon and is defined as $\tau=\frac{Q^{2}}{4 M^{2} c^{2}}$ [76].

$$
\left(\frac{d \sigma}{d \Omega}\right)_{\substack{\text { point } \\ \text { spin } 1 / 2}}=\left(\frac{d \sigma}{d \Omega}\right)_{\text {Mott }} \cdot\left[1+2 \tau \tan ^{2} \frac{\theta}{2}\right]
$$

The interaction described in the Mott cross section equation is mediated by a single photon and is electromagnetic in nature. For an electromagnetic interaction conducted at a low resolution, there is an agreement between the measured cross section and the theoretical Mott cross section. This agreement is maximized when in the limit of $|\boldsymbol{q}| \rightarrow 0$ for scattering 
events of electrons off of a target nuclei. As $|\boldsymbol{q}|$ climbs further from zero and the resolution of probe grows, the experimentally measured cross sections will begin to differ from the Mott cross section, systematically decreasing [76]. The comparison of the Mott calculated cross section to experimentally measured cross section for a $Q^{2}$ of $2.5 \mathrm{GeV}^{2} / \mathrm{c}^{2}$ is shown in figure 1.2. Increasing the $|\boldsymbol{q}|$ of an interaction reduces the size of the wavelength of the virtual photon that mediates the electromagnetic interaction between the electron and target nuclei and therefore increases the resolution of the probe. The wavelength of this virtual photon is inversely proportional to $|\boldsymbol{q}|$, and can be described by the following: $\lambda=\frac{\hbar}{|\boldsymbol{q}|}$ [76]. Increasing the amount of momentum transferred in an electromagnetic reaction allows one to study deeper into the nucleus. The act of probing deeper into the nucleus or nucleon allows for the study of the substructure of the target.

Studying the internal structure of a nucleus with the electromagnetic interaction requires increasing the momentum transferred. Pushing $|\boldsymbol{q}|$ to be comparable with the mass of a nucleon adds more complexity to the details of the scattering interaction. At the appropriate levels of $|\boldsymbol{q}|$ to study the nucleons in the nucleus, the Mott cross section equation requires modifications to include additional factors that incorporate information about the target. The Rosenbluth formula is based on the Mott cross section and embraces target recoil, magnetic moment, and charge and current distributions. Povh [76] writes the Rosenbluth formula as:

$$
\left(\frac{d \sigma}{d \Omega}\right)=\left(\frac{d \sigma}{d \Omega}\right)_{M o t t} *\left[\frac{G_{E}^{2}\left(Q^{2}\right)+\tau G_{M}^{2}\left(Q^{2}\right)}{1+\tau}+2 \tau G_{M}^{2}\left(Q^{2}\right) \tan ^{2} \frac{\theta}{2}\right]
$$

Equation 1.9 contains $G_{E}^{2}\left(Q^{2}\right)$ and $G_{M}^{2}\left(Q^{2}\right)$, the electric and magnetic form factors. These form factors depend on $Q^{2}$, and this measured $\mathrm{Q}^{2}$ dependence provides information on the radial charge distributions and magnetic moments of the scattering participants [76]. For the instance of $Q^{2} \rightarrow 0$, the values of $G_{E}^{2}(0)$ and $G_{M}^{2}(0)$ are physically important.

$$
\begin{array}{ll}
G_{E}^{P}\left(Q^{2}=0\right)=1 & G_{E}^{n}\left(Q^{2}=0\right)=0 \\
G_{M}^{P}\left(Q^{2}=0\right)=2.79 & G_{M}^{n}\left(Q^{2}=0\right)=-1.91
\end{array}
$$




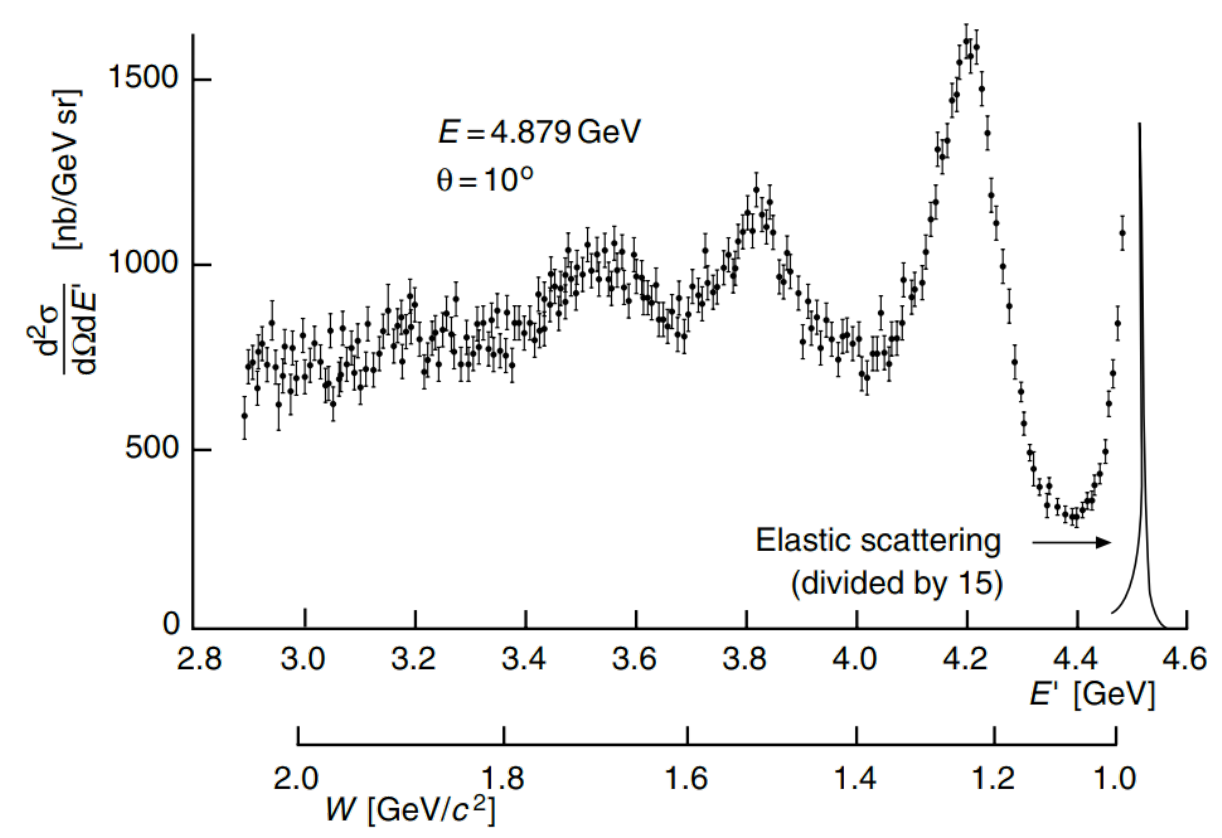

Figure 1.3: Electron-proton scattering for incident energy of $4.9 \mathrm{GeV}$ and scattering angle of $10^{\circ}[19]$.

The $G_{E}^{2}(0)$ corresponds to the electric charge of the target. $G_{M}^{2}(0)$ is simplified to the magnetic momentum normalized by the nuclear magneton. The result of $G_{E}^{2}(0)$ and $G_{M}^{2}(0)$ for the proton and neutron are shown in equation 1.10[76]. There were many experiments at SLAC that studied the $Q^{2}$ dependence of these form factors in the early seventies. The results from these form factor experiments determined that $G_{E}^{p}\left(Q^{2}\right)=\frac{G_{M}^{P}\left(Q^{2}\right)}{2.79}=\frac{G_{M}^{n}\left(Q^{2}\right)}{-1.91}=$ $G^{\text {dipole }}\left(Q^{2}\right)$. Where $G^{\text {dipole }}\left(Q^{2}\right)$ is a dipole fit that describes the form factors very well[76].

\subsection{Deep inelastic scattering}

The first generation of electron scattering experiments achieving a significantly large $|\boldsymbol{q}|$ used a linear accelerator with a $25 \mathrm{GeV}$ maximum beam energy, and following generations increased the total interaction energy to substantially higher thresholds. At these high incident beam energies, individual resonances cannot be separated in the invariant mass spectrum above $2.5 \mathrm{GeV}$. Observations made into this convoluted region of invariant mass spectrum has shown that many strongly interacting particles are produced, known as hadrons. Scattering interactions that generate these hadrons are considered to be inelastic. 
Figure 1.3 contains the invariant mass spectrum for an electron scattering from a proton target for an incident energy of $4.9 \mathrm{GeV}$ and an angle of $10^{\circ}$ [19]. These results are from an experiment at Deutsches Elektronen-Synchrotron (DESY) published in 1968. The elastic scattering peak is scaled down by a factor of 15 to provide an appropriate scaling of the complete spectrum. As $\nu$ increases or the scattered electron energy decreases relative to the incident energy, the invariant mass of the scattering interaction increases. As $W$ rises, the resonance begins to convolute together. This behavior is indicative of reaching a new threshold.

Inelastic scattering events contain the possibility of producing strongly interacting particles causing an increase in the complexity of a scattering interaction. In order to create an inelastic event, the wavelength of the virtual photon has to be comparable to the radius of the struck nucleon. Increasing the amount of transferred momentum so that $Q^{2} R^{2} \gtrsim 1$ will increase the resolution of the probe to a level that allows for the interaction to be with the charge constituents within the nucleon. When the scattering event probes the fundamental elements of a nucleon, the scattering process is titled deep inelastic scattering (DIS). Due to the increase in complexity, an additional degree of freedom has to be introduced into the scattering cross section formalism. Modifying the Rosenbluth formula to include the inelastic scattering structure functions $F_{1}\left(Q^{2}, \nu\right)$ and $F_{2}\left(Q^{2}, \nu\right)$ evolves the Rosenbluth formula to contain the needed complexity of an inelastic event. These modifications are shown in equation 1.11. The $F_{1}$ and $F_{2}$ structure functions provide the details for describing the internal composition of the nucleon [76]. For elastic scattering events $2 M \nu-Q^{2}=0$, this forces only one kinematic parameter to vary freely. However for inelastic scattering events $2 M \nu-Q^{2}>0$, this creates an additional free parameter and is the reason for the $F_{1}$ and $F_{2}$ structure functions being functions of both $Q^{2}$ and $\nu$.

$$
\frac{d^{2} \sigma}{d \Omega d E^{\prime}}=\left(\frac{d \sigma}{d \Omega}\right)_{M o t t}\left[\frac{F_{2}\left(Q^{2}, \nu\right)}{\nu}+\frac{2 F_{1}\left(Q^{2}, \nu\right)}{M} \tan ^{2} \frac{\theta}{2}\right] .
$$




\subsubsection{Scaling}

The Bjorken scaling variable, $x_{B}$ or $x$, is a dimensionless quantity that measures the inelasticity of a scattering process and is defined as $x \equiv \frac{Q^{2}}{2 M \nu}$. Measurements for the DIS $F_{2}$ structure function are displayed in figure 1.4. This plot displays results of $F_{2}$ as a function of $x$ and $Q^{2}$. The $x$ dependence of $F_{2}$ is strong and shows that $F_{2}$ will decrease as $x$ increases. However, at a constant value of $x$, the dependence of $Q^{2}$ on $F_{2}$ is weak for moderate values of $x$. This phenomenon of a solo dependence on $x$ was known as scaling. This scaling was observed to be present for scattering interactions with $Q^{2}>2 \mathrm{GeV}^{2}$ and $\nu>2.5 \mathrm{GeV}$ [16]. In the Bjorken limit, $\nu \rightarrow \infty$ and $Q^{2} \rightarrow \infty$, the deep inelastic structure functions can be described as functions of only $x$ instead of $Q^{2}$ and $\nu$ [25].

\subsubsection{Quark Parton Model}

In the case of DIS off of a proton, the electron probe is used to explore the exclusive internal structure of the proton, its constituents. In 1969, Feynman assumed the internal make up of the proton was that of point-like partons, the basis of the parton model [25, 58]. As part of this model, the impulse approximation makes an assumption that the duration of the interaction between the mediating photon and parton is relatively short, allowing for the interaction between individual partons to be neglected. Thus in a DIS interaction, the partons can be described as quasi-free, with minimal internal interactions. Under this understanding, an electron-nucleon DIS interaction would characterize the properties and motions of the partons that form the struck nucleon[58].

The characteristics (the motions and properties) of the partons are formalized into a parton distribution function $f_{i}\left(x_{B}\right)$ [76]. The relationship for the parton distribution function with the $F_{2}$ structure function is shown in equation 1.12. The $F_{1}$ structure function is the DIS equivalent to the magnetic form factor from equation 1.10, and will vanish for scattering from spin zero particles [76]. Figure 1.5 shows the linear relationship between $F_{1}$ and $F_{2}$ by

plotting the ratio of $\frac{2 x F_{1}}{F_{2}}$ as a function of $x$. This data from SLAC helped confirm theories from C. G. Callan Jr. and David J. Gross that the partons that are found in the nucleons 


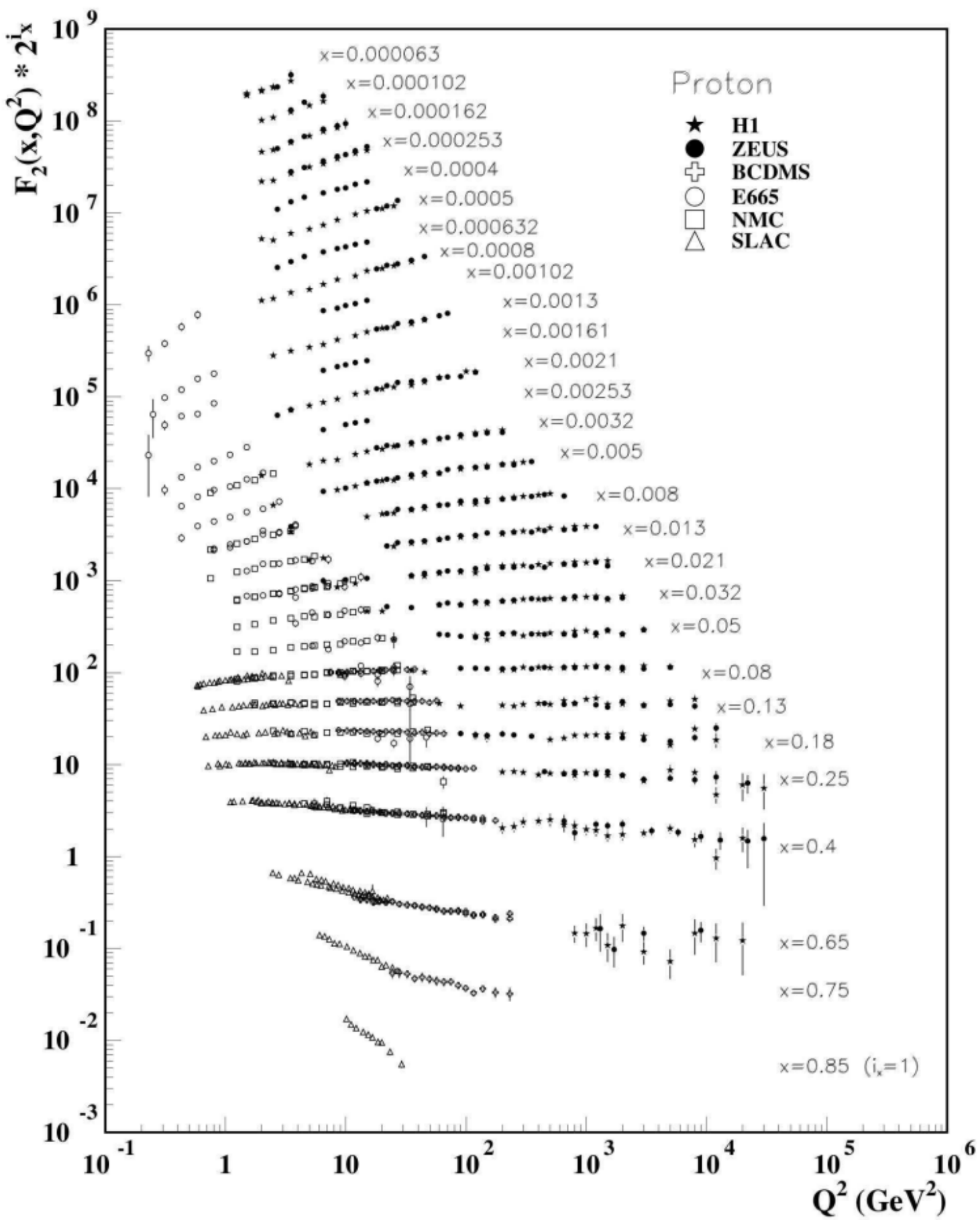

Figure 1.4: Measurements of the proton structure function $F_{2}\left(x, Q^{2}\right)$ for different $x$ settings[38]. The $Q^{2}$ independence shows that there is nothing smaller than quarks. 


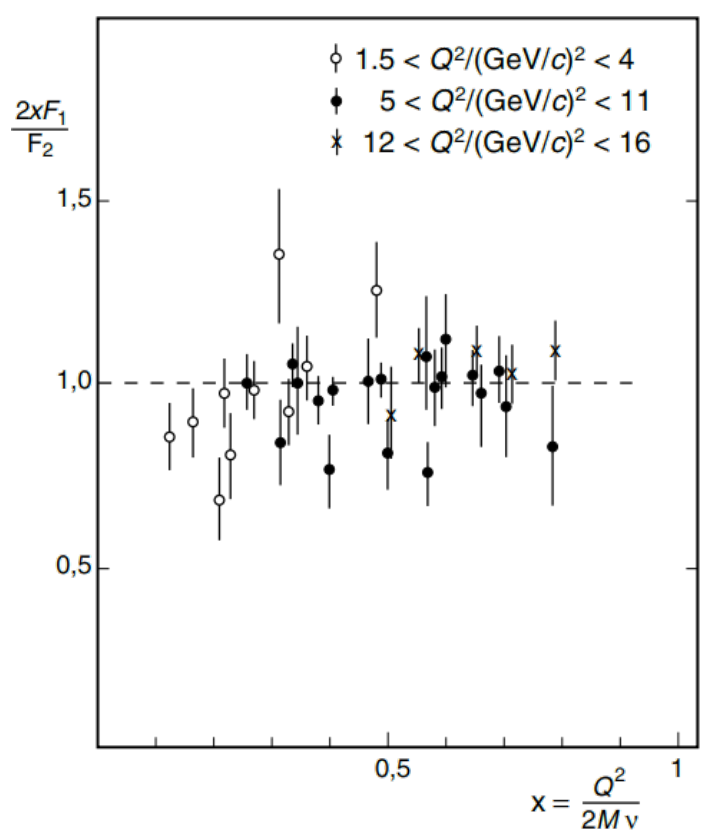

Figure 1.5: Data from SLAC, showing the ratio of the structure functions $2 x \cdot F_{1}(x)$ and $F_{2}(x)$ vs. $x[76,75]$.

of a nucleus are spin $1 / 2[28,41]$. The relationship between $F_{1}$ and $F_{2}$ is known as the Callan-Gross relation [76]. This relationship can be seen in equation 1.13.

$$
\begin{gathered}
F_{2}(x)=x \Sigma_{i} e_{i}^{2} f_{i}(x) \\
F_{1}(x)=\frac{1}{2 x} F_{2}(x) \\
F_{2}(x)=x \cdot \Sigma_{f} z_{f}^{2}\left(q_{f}(x)+\bar{q}_{f}(x)\right)
\end{gathered}
$$

The electromagnetic interaction that occurs during a scattering event happens between two charged bodies. The electron carries a charge of $-e$ and the proton carries a charge of $+e$. The partons that makeup the proton or neutron must carry a total charge equal to the charge of the proton or neutron. Using DIS scattering from electron, neutrino, and muon beams, the amount of charge carried by the partons was determined by using equation 1.14 $[76,41,28]$. 


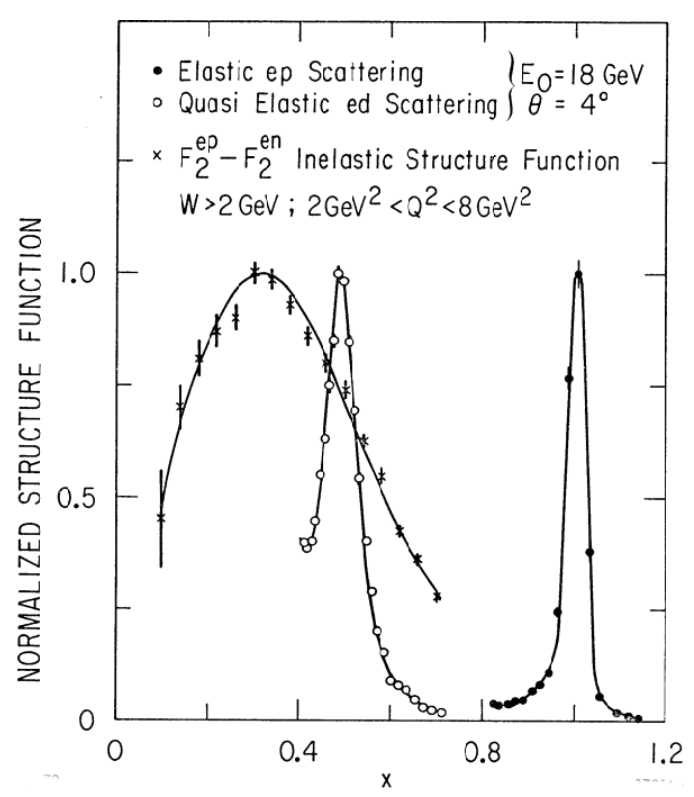

Figure 1.6: Structure function results measured from three lepton-nucleon scattering experiments [16].

Figure 1.6 from Atwood et. al. (1982), contains data on three unique experiments plotting structure function results against $x$. Solid dots are elastic electron-proton scattering at an incident energy of $18 \mathrm{GeV}$ and scattered angle of $4^{\circ}$. The elastic peak at $x$ of 1 is due to the scattering event happening elastically off the entire proton. The open points are quasi-elastic scattering from $\mathrm{D}$. The peak is located at 0.5 in $x$ because the scattering event happens from the proton and neutron, which individually contain only half of the mass of the complete deuteron. The data represented by an ' $x$ ' displays result from an inelastic electron scattering measurement. The data plotted is the difference between the two nucleon structure functions. The peak is located at one-third. The location of the peak at one-third demonstrates that the struck constituents of the nucleon have a mass approximately one-third of the nucleon and there exist three constituents inside the nucleon with equal mass[16, 76].

Nuclear physicists of the mid 19th century changed the understanding of the building blocks of nature by discovering fundamental constituents of the protons and neutrons. It was unearthed that these partons have an electric charge, spin of $1 / 2$, and some mass. Due to these partons having these properties, they can be identified as quarks from Gell-Mann's symmetry scheme, the eightfold way. This theory was based on the SU(3) mathematical 


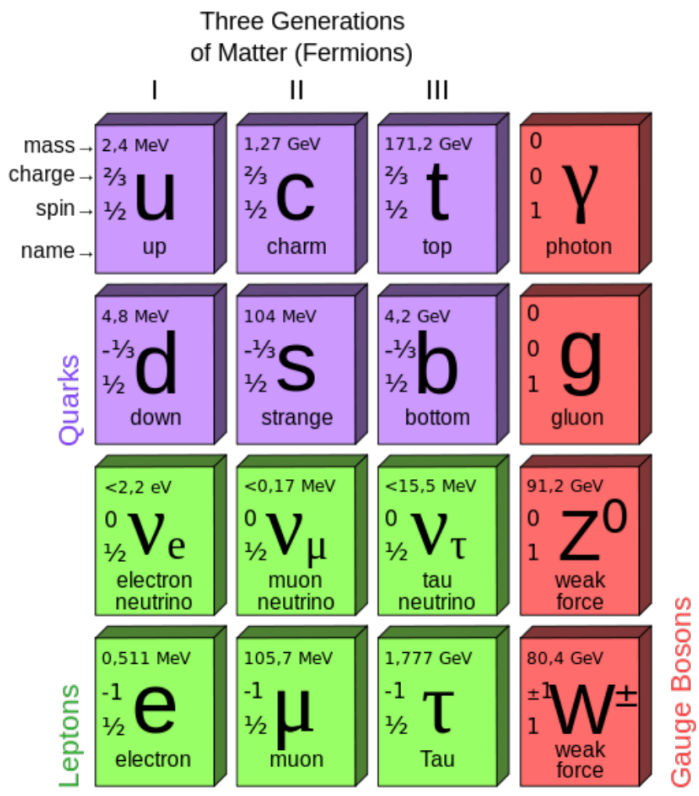

Figure 1.7: Elementary particles including leptons, quarks, and bosons with mass, charge, and spin [68].

symmetry [47, 44]. Through nuclear and high energy experiments six quarks have been discovered[24]. A table of the elementary particles is shown in figure 1.7.

A scattering interaction between a lepton probe and a target nucleus probes the structure of the target. A DIS interaction delves inside the nucleus to observed the nucleons and their quarks and gluons. The European Muon Collaboration (EMC) used DIS experiment to study the internal structure of a few targets. Their use of DIS in 1983 discovered a new phenomenon defined as the EMC effect [76, 52]. 


\section{Chapter 2}

\section{EMC Effect}

\subsection{European Muon Collaboration}

The European Muon Collaboration (EMC) performed a deep inelastic measurement with 120-280 GeV muons on Fe, H, and D targets to begin a comprehensive study of muon scattering [64, 72]. The EMC used muons to reach their goal of achieving interactions at a large $Q^{2}$ [80]. The EMC studied the per nucleon normalized Fe/D structure function ratio versus the Bjorken scaling variable, $x$. The EMC expectation for this ratio originally was unity for $x$ between 0.05 and 0.7 and would deviate at higher $x$ due to Fermi smearing[52]. The reasoning for this expectation was the belief that at large magnitude of $Q^{2}$ the interaction between protons and neutrons would not contribute to the total structure function of the nucleus. This was the understanding because the binding energy of a few $\mathrm{MeV}$ would not interfere with the GeV scale of the DIS interaction [33]. The expected structure function for a nucleus could be written as:

$$
F_{2}^{A}=N F_{2}^{N}+Z F_{2}^{P}
$$

In this quasi-free nucleon picture, the nucleons are used to build up the nuclear structure $\left(F_{2}^{A}\right)$ by summing up the neutron structure functions $\left(F_{2}^{N}\right)$ with the proton structure functions $\left(F_{2}^{P}\right)$ for each nucleon. Figure 2.1 displays the results from the EMC ratio comparison of Fe and D. The $\frac{A}{D}$ structure function ratio showed an unexpected downward slope. This phenomenon was titled the EMC effect. This finding demonstrated to the EMC that their 


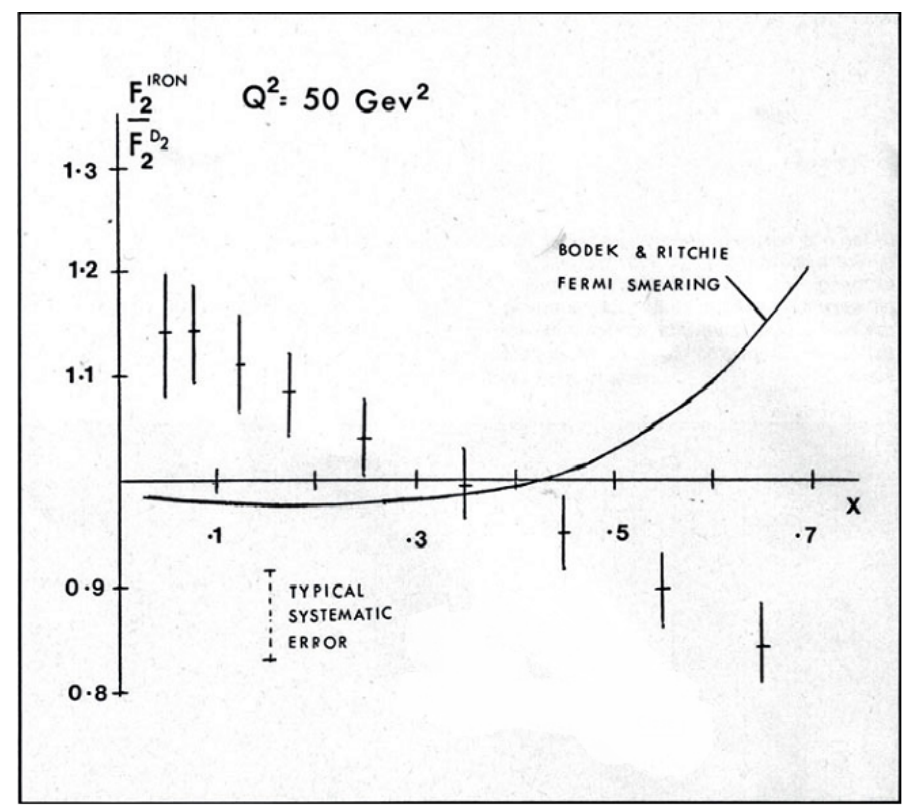

Figure 2.1: Graph of the ratio of A/D structure functions vs $x$ from the EMC. [52, 12].

understanding of the nucleus was incorrect. A nucleon's structure function and thereby, the constituent quark distributions are altered by the structure of the nuclear medium.

\subsection{Ratios of Cross Sections and Structure Functions}

In chapter one, we defined the inelastic cross section in equation 1.11.

$$
\sigma^{A}=\frac{4 \alpha^{2} E^{\prime 2}}{Q^{4}}\left[2 \frac{F_{1}^{A}(x)}{M} \sin ^{2} \frac{\theta}{2}+\frac{F_{2}^{A}(x)}{\nu} \cos ^{2} \frac{\theta}{2}\right]
$$

In figure 2.1, the EMC collaboration analyzed the ratio of $F_{2}$ structure functions. The per nucleon cross section of two different nuclei can be reduced to the ratio of the $F_{2}$ structure functions.

$$
\frac{\sigma_{A_{2}}}{\sigma_{A_{1}}}=\frac{F_{2}^{A_{2}}}{F_{2}^{A_{1}}}
$$

The reduction of the ratio of two nuclei begins by using the ratio of longitudinal and transverse cross sections as a function of $F_{1} / F_{2}$.

$$
R=\frac{\sigma_{L}}{\sigma_{T}}=\left(1+\frac{\nu^{2}}{Q^{2}}\right) \frac{M F_{2}}{\nu F_{1}}-1
$$


The ratio of two unique per nucleon cross sections is:

$$
\frac{\sigma_{A_{2}}}{\sigma_{A_{1}}}=\frac{F_{2}^{A_{2}}}{F_{2}^{A_{1}}} \frac{\left[1+2 \frac{\nu F_{1}^{A_{2}}}{M F_{2}^{A_{2}}} \tan ^{2} \frac{\theta}{2}\right]}{\left[1+2 \frac{\nu F_{1}^{A_{1}}}{M F_{2}^{A_{1}}} \tan ^{2} \frac{\theta}{2}\right]}
$$

Where $A_{1}$ and $A_{2}$ denote the different nuclei. Using the definition of $R$ in equation 2.4 , the per nucleon cross section ratio of $A_{1}$ and $A_{2}$ can be simplified to equation $2.3[12,80]$. The simplification of the cross section ratio to the structure function ratio is based on the use of $R$. $R$, the longitudinal and transverse cross section ratio has been studied extensively for many nuclei. The measurements of $R$ have shown little dependence on the number of nucleons [12].

The $x$ spectrum of a per nucleon cross section ratio of some nucleus with A nucleons and $\mathrm{D}$ also known as an $A / D$ ratio or an EMC ratio is divided into 4 different regions.

- For $x<0.1$, the shadowing region has an EMC ratio that shows a decline of the nuclear structure functions. A coupling of the photon to strongly interacting quarks causes this feature [76].

- The anti-shadowing region of the $x$ spectrum lies at $0.1 \leq x<0.3$. The results of DIS experiments show an EMC ratio slightly larger than unity in this region. This increase is caused by constructive interference among the multi-scattering amplitudes in the nucleus [26].

- $x$ between 0.3 and 0.7 is the EMC effect region. This region will be discussed further in this chapter.

- For $x>0.7$, the EMC ratio grows rapidly above unity. This region is the Fermimotion region. The motion of the nucleons inside a nucleus creates a distribution of the nucleons' momentum. The convolution between the nucleons' structure function and momentum distribution form the nuclear structure function. This causes the nuclear structure function of an $\mathrm{A}>2$ nucleus to rise quickly compared to a D nucleus $[33,76]$. 


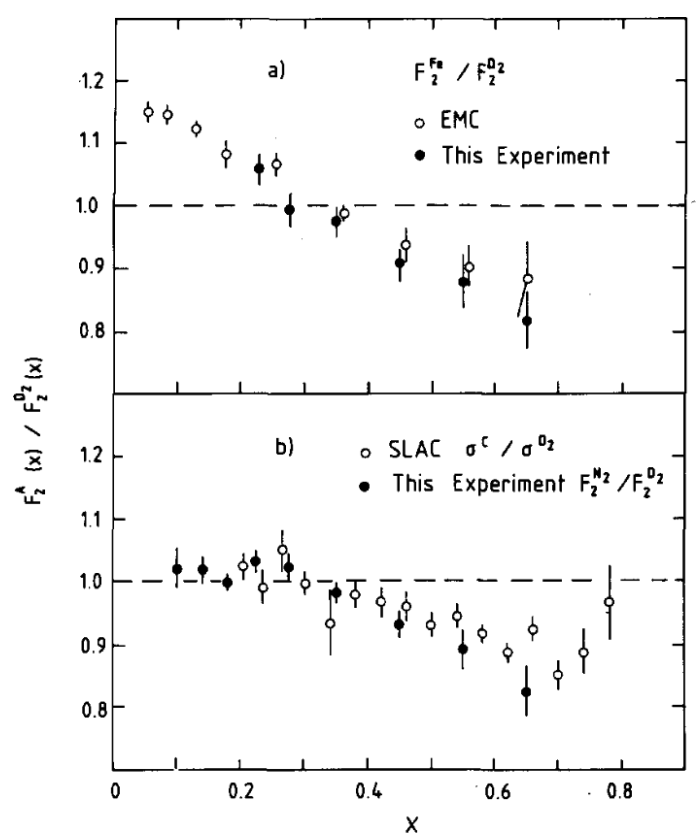

Figure 2.2: EMC effect from the BCDMS collaboration [18].

\section{$2.3 \quad$ EMC Experiments}

\subsubsection{Experiments at CERN}

EMC The EMC published results from muon beam experiments in 1981-1983 [11, 12, 13, 14]. The EMC used data from this group of experiments to form the first EMC ratios, shown in 2.1. The experiments used muon beams of 120 to $280 \mathrm{GeV}$ to extract nuclear and nucleon structure functions from $\mathrm{Fe}, \mathrm{D}$ and $\mathrm{H}$ targets. The use of multiple incident beam energies allowed these experiments to have a $Q^{2}$ for $x$ of 0.05 between 8 and $20 \mathrm{GeV}^{2}$ and a $Q^{2}$ for $x$ of 0.65 between 35 and $200 \mathrm{GeV}^{2}$ [14]. Throughout this run of experiments, the EMC used the EMC forward detector but the experiments were conducted at different times causing a rise in the total uncertainties for the EMC ratios[12]. After publishing the results for the EMC effect, the EMC conducted another round of experiments for two reasons. First, the EMC focused on decreasing the systematic uncertainties that were seen in the first EMC effect analysis. They also want to expand their knowledge of the EMC effect on more nuclei $[10,33]$. This included measuring muon scattering on carbon, copper, and tin [10]. 
BCDMS The Bologna-CERN-Dubna-Munich-Saclay (BCDMS) collaboration at CERN continued the study of the EMC effect by comparing their measurement of the cross section of $\mathrm{N}$ and $\mathrm{Fe}$ to $\mathrm{D}$. This experiment used a $40 \mathrm{~m}$ long iron toroid magnet with 8 modules consisting of scintillators and multiwire proportional chambers [18]. The data collected from this spectrometer is shown in figure 2.2. The BCDMS results show a good comparison from their EMC effect for Fe and the results from the EMC in plot a. In plot 'b', BCDMS collaboration compare their nitrogen EMC results to SLAC's carbon EMC result [18, 72].

NMC In the winter of 1985, the New Muon Collaboration (NMC) purposed to used the muon beam at CERN to expand the understanding of the A dependence for the EMC ratios at low- $x$ and to understand the $Q^{2}$ dependence of the EMC ratios. Along with the EMC ratios, the NMC also wanted to improve the current measurements for the neutron structure function, $F_{2}^{n}$, and the neutron to proton structure function ratio, $\left(F_{2}^{n} / F_{2}^{p}\right)$ [70]. This experiment consisted of completing muon scattering on solid targets of $\mathrm{Be}, \mathrm{C}, \mathrm{Al}, \mathrm{Ca}$, $\mathrm{Fe}, \mathrm{Sn}$, and $\mathrm{Pb}$. The data for this experiment covered a kinematic range in $x$ of 0.01 to 0.8 , and in $Q^{2}$ from 2 to $70 \mathrm{GeV}^{2}[5]$. The NMC concluded the $Q^{2}$ dependence for the EMC ratios is small and the dependence of A for the EMC effect is approximately logarithmic $[5,33]$.

\subsubsection{Experiments at SLAC}

Scientists at the Stanford Linear Accelerator Center (SLAC) extracted EMC ratios for many nuclei including; ${ }^{4} \mathrm{He},{ }^{9} \mathrm{Be},{ }^{12} \mathrm{C},{ }^{27} \mathrm{Al},{ }^{40} \mathrm{Ca},{ }^{56} \mathrm{Fe},{ }^{108} \mathrm{Ag}$, and ${ }^{197} \mathrm{Au}$. This experiment used an electron beam of 8 to $24.5 \mathrm{GeV}$. The data spanned a large range of $x$, from 0.089 to 0.8 , and $Q^{2}$, from 2 to $15 \mathrm{GeV}^{2}$ to extract cross sections ratios. The EMC ratios were extracted by counting the electrons detected by the SLAC 8-GeV/c magnetic focusing spectrometer [45]. The EMC ratios for the eight different nuclear targets are shown in figure 2.3 .

The analysis of these ratios revealed the magnitude of the EMC effect, taken to be the A/D ratio at $x=0.6$, was found to be different for the various nuclei, and roughly scaled with the 


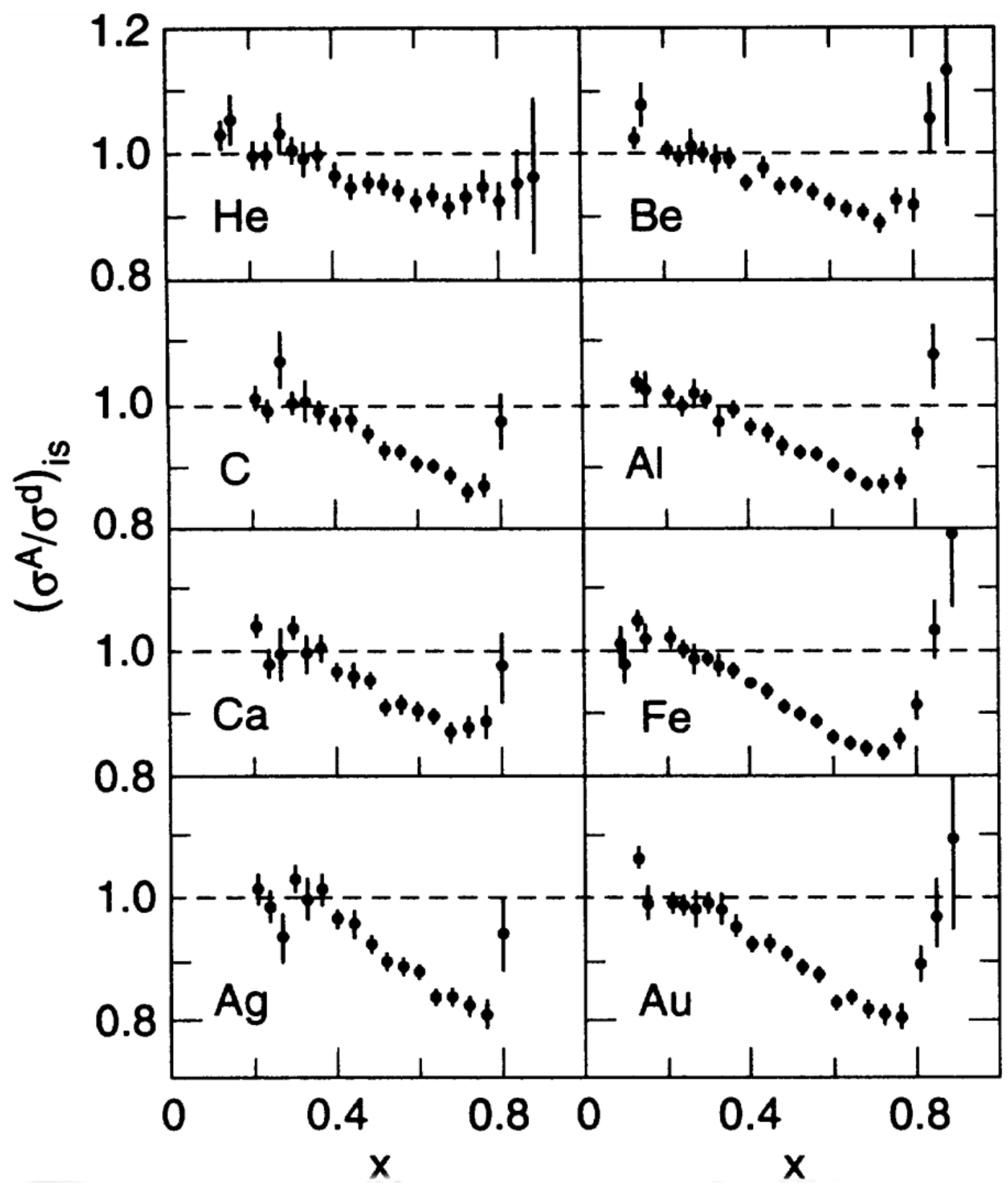

Figure 2.3: EMC ratios from SLAC. The plot shows the $Q^{2}$ average cross section ratios with isoscalar corrections for different nuclei [45]. 


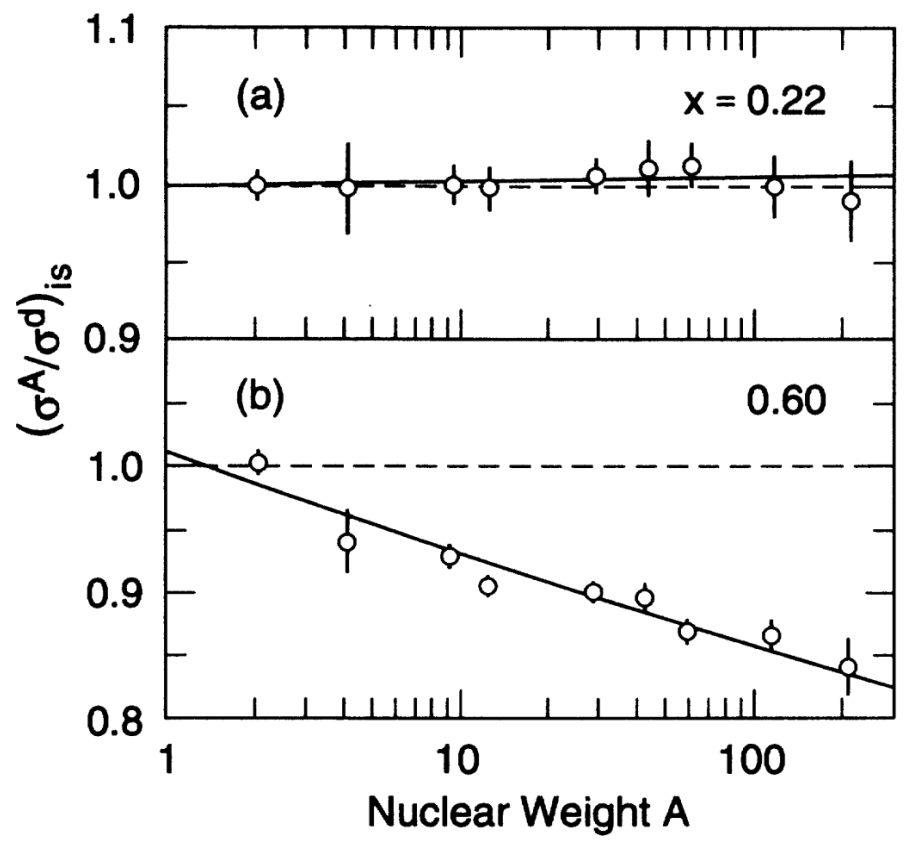

Figure 2.4: The dependence of the atomic mass number on the EMC effect[45].

size or density of the nuclei. Figure 2.4, shows the EMC effect magnitude as a function of the nuclear weight of the targets. It demonstrates an agreement with data from the NMC, that the EMC effect's dependence on the nuclear mass number, A, is approximately logarithmic $[33,45,80]$.

\subsubsection{HERMES at DESY}

The High-Energy Radiation Megavolt Electron Source (HERMES) collaboration used the Hadron-Electron Ring Accelerator (HERA) at Deutsches Elektronen-Synchrotron (DESY), German Electron Synchrotron, to study the DIS cross section ratios of ${ }^{3} \mathrm{He},{ }^{14} \mathrm{~N}$, and ${ }^{84} \mathrm{Kr}$ with respect to D [2]. Data were collected at $x$ kinematics ranging from 0.010 and 0.65 with $Q^{2}$ varying between 0.5 and $15 \mathrm{GeV}^{2}[2]$. The HERMES collaboration used a $27.5 \mathrm{GeV}$ positron beam to scatter off of gaseous targets into the HERMES forward angle spectrometer.

\subsubsection{Experiments at Jefferson Lab}

Experiments at Thomas Jefferson National Accelerator Facility (JLab) produced two notable EMC ratio results. In 2006, an experiment designed to study the scaling of the 
structure functions of the target nucleus produced data for the extraction of EMC ratios for $\mathrm{C}, \mathrm{Fe}$, and $\mathrm{Au}$. The kinematics of this experiment produced data in the resonance region with a $Q^{2} \approx 4 \mathrm{GeV}^{2} / \mathrm{c}^{2}$ and $1.2<W^{2}<3 \mathrm{GeV}^{2} / \mathrm{c}^{2}$. These data are shown in figure 2.5 compared with data from SLAC and BCDMS.

$$
\xi=\frac{2 x}{\sqrt{1+\frac{4 M^{2} x^{2}}{Q^{2}}}}
$$

The SLAC and BCDMS experiments took data in the DIS region with a $W^{2}>3 \mathrm{GeV}^{2} / \mathrm{c}^{2}$. The $Q^{2}$ value of the data needs to be accounted for in a comparison made between these three experiments. This has been done by using the Nachtmann variable $\xi$, defined in equation 2.6 [9]. The results show that the EMC ratio in the resonance region matches the same ratio from the DIS region and therefore DIS structure functions information can be extracted from the resonance region [80].

In 2009, results from another Jefferson lab experiment were published describing the EMC effect in light nuclei. This experiment measured the inclusive cross section from $\mathrm{D},{ }^{3} \mathrm{He},{ }^{4} \mathrm{He}$, ${ }^{9} \mathrm{Be}$, and ${ }^{12} \mathrm{C}$ for $x$ between 0.3 and 0.9 , with $Q^{2} \approx 3-6 G e V^{2}$ [79]. This experiment provided the first results for ${ }^{3} \mathrm{He}$ EMC ratios for high $x$. Also, results from this experiment showed that the nuclear dependence of the EMC effect may depend on the local nuclear environment [79]. Previous results showed a logarithmic dependence of the EMC effect on A. The EMC

results for ${ }^{9} \mathrm{Be}$ showed the dependence on $\mathrm{A}$ or average density scaling picture was wrong. Figure 2.6 displays the outlying result from ${ }^{9} \mathrm{Be}[79]$.

\subsection{EMC Theory and Models}

Since the observation of the EMC effect, there have been numerous amounts of work conducted on the theories to describe these EMC ratios. The models attempt to characterize both the nuclear and nucleon structure functions for the entire range of $x$ from 0.0 to 1.0. This section will briefly discuss the basic idea of a few EMC models. 


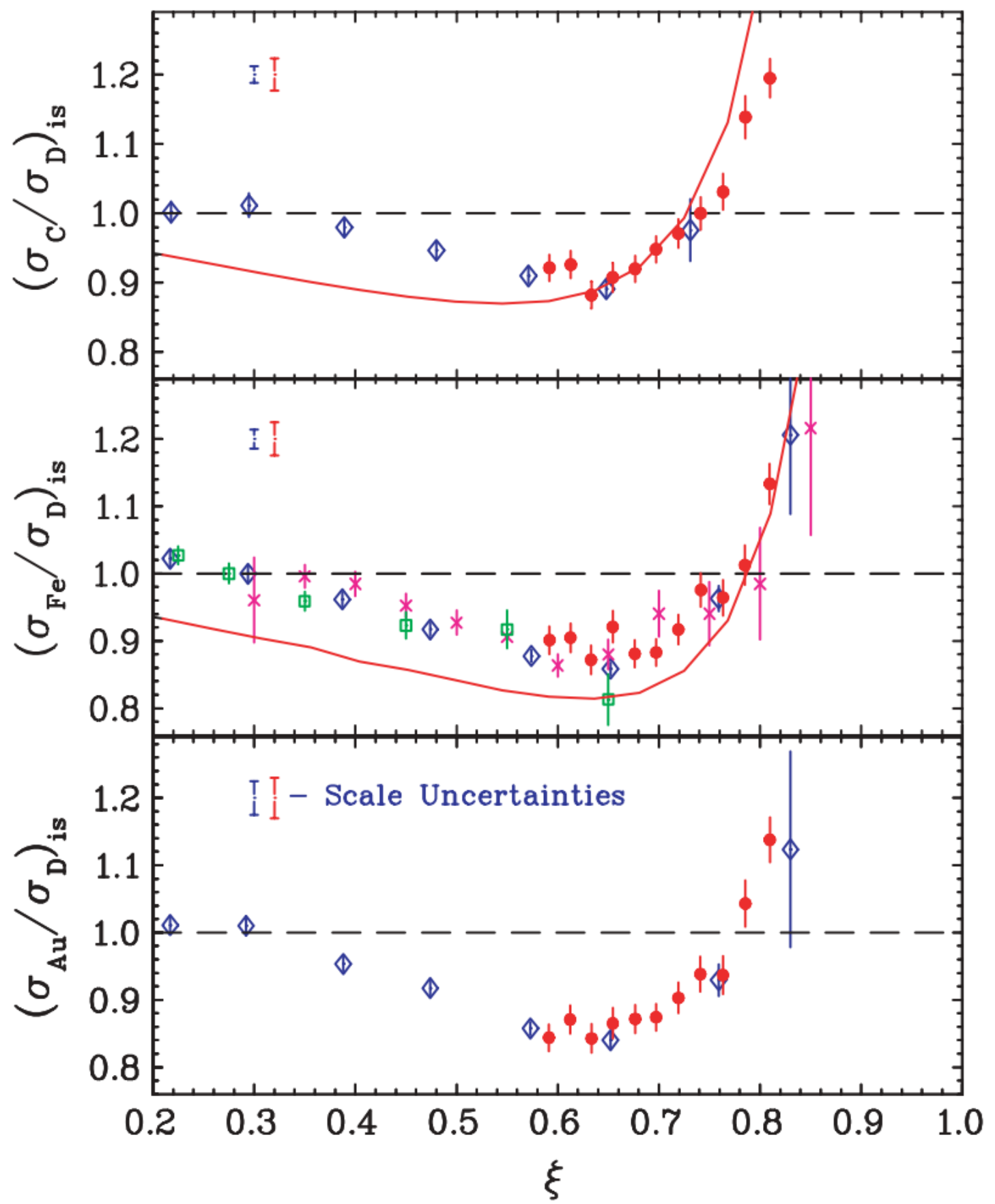

Figure 2.5: Ratio of nuclear to D per nucleon cross sections corrected for neutron excess[9]. The JLab data in red is compared with SLAC data in Blue [45] and BCDMS data in green [18]. The red lines are model extractions from [48]. 


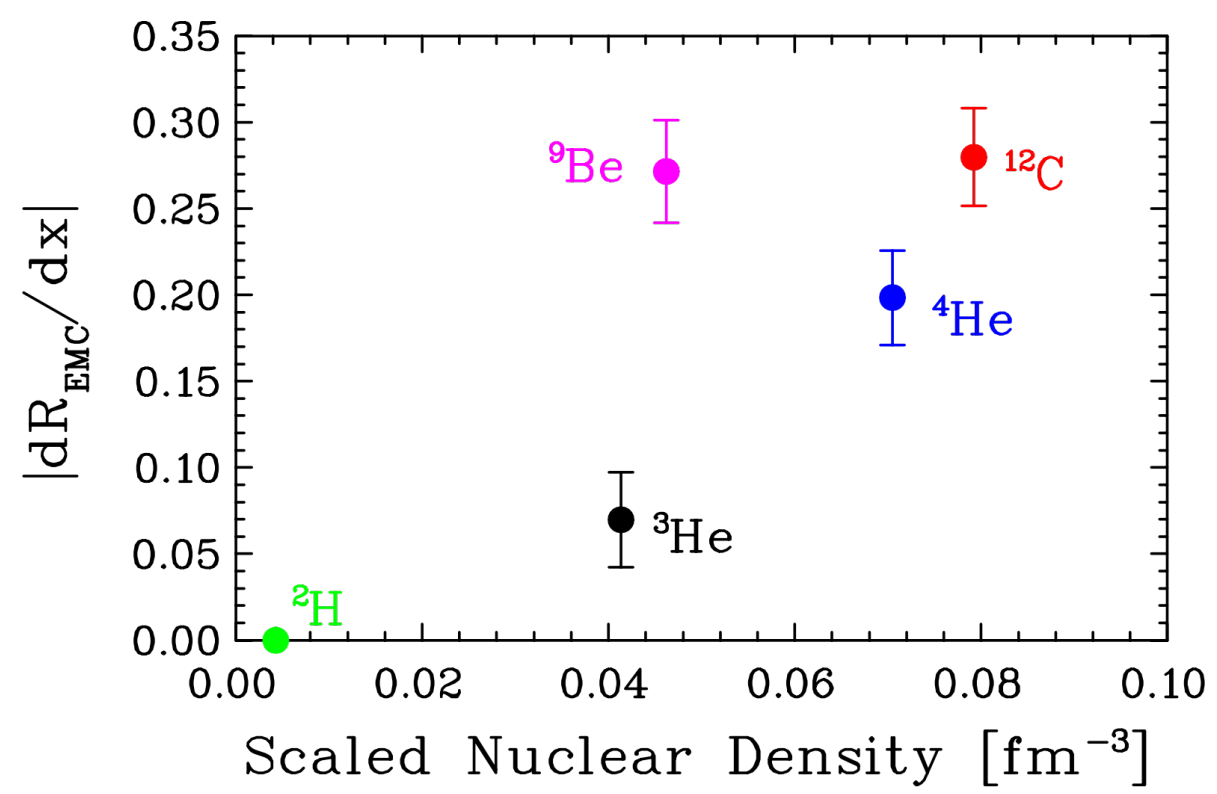

Figure 2.6: Isoscalar EMC effect as function of nuclear density [79].

\subsubsection{Multiquark Cluster}

The multiquark cluster model discussed by K. M. Hanna et. al. [50] and R. L. Jaffe [55], states that it is possible to form color singlet quark clusters inside of a dense nucleus. These quark clusters can contain $3 \mathrm{~N}$ quarks $(3,6,9 \ldots)$ [72]. These quark clusters have the possibility to contain the momentum of multiple nucleons [33]. Because of the overall momentum of these clusters, the multiquark cluster models make predictions for high $x$, but these clusters are not understood enough to make predictions at low $x[21,43,33,50]$.

\subsubsection{Nuclear Binding}

Describing the EMC effect via nuclear binding was first attempted by Akulinechev et. al. [3] and Dunne and Thomas [36]. For the nuclear binding model, an average nucleon has a momentum and separation energy defined as $\vec{p}$ and $\langle\epsilon\rangle$ respectively [72]. Including the separation energy in the defining of the momentum of the post scattering A-1 system causes a manipulation in the value of $x$. $x$ becomes $x^{\prime}=\frac{Q^{2}}{2 p^{\prime} \cdot q}$, where $p^{\prime}$ is $(\mathrm{M}+\epsilon, \vec{p})[72]$. The modification of the momentum through nuclear binding allows this EMC model to explain 
the EMC effect and the sharp rise in the Fermi motion area but fails to correctly describe the rise in the anti-shadowing region around $x=0.2[20,21,33]$.

\subsubsection{Medium Modification}

Smith and Miller [81] claim that measurements of nuclear observable could be explained by modifications of the nuclear structure due to the medium. The medium is filled with external fields created by the surrounding nucleons. These fields modify the quark waveform of a single nucleon. Using medium modifications C. Cloet, W. Bentz, and A. W. Thomas [29] were able to describe the EMC effect for a collection of nuclear targets and calculate the correct A dependence of the EMC effect.

\subsubsection{Rescaling}

Nachtmann and Pierner [69] and Close et al [30] discovered a way to relate the DIS structure functions of Fe and D with scaling variables. They found that by using a relative shift in the scale of the $Q^{2}$ value that $F_{2}^{F e}\left(Q^{2}\right)=F_{2}^{D}\left(\xi Q^{2}\right)$ [43]. Both teams proposed that as nuclei get heavier their quarks are bound in an area larger compared to the confinement area for a free nucleon. This dynamic rescaling model is applicable for $0.2<x<0.8$, but does not match the EMC ratio for the Fermi motion region with $x>0.8$ [21, 30, 43, 69].

The models discussed here are only a small subset of the models that have been used to describe the EMC ratios. The downfall for most of these models is the inability to consistently predict the EMC ratio for all nuclear targets and the entire range of $x$ in DIS. In order to better constrain the EMC effect and gain a better understanding of DIS, we need to conduct more experiments directly targeting specific regions of the complex problem.

\subsection{MARATHON}

Experiment E12-010-102, MARATHON (MeAsurement of the $F_{2}^{n} / F_{2}^{p}, d / u$ RAtios and A=3 EMC Effect in Deep Inelastic Electron Scattering Off the Tritium and Helium MirrOr Nuclei), will use deep inelastic scattering off of the mirror nuclei ${ }^{3} \mathrm{H}$ and ${ }^{3} \mathrm{He}$ to measure the EMC 
effect for both ${ }^{3} \mathrm{H}$ and ${ }^{3} \mathrm{He}$, to determine the ratio of the neutron to proton inelastic structure functions, and to find the ratio of the down to up quark distributions in the nuclei [32].

The MARATHON experiment has provided DIS data to determine the EMC effect for the two $A=3$ mirror nuclei. Previous experiments to measure the ${ }^{3} \mathrm{He}$ EMC effect were able to gather data at low and medium $x$ for DIS kinematics, and data in the resonance region for higher $x$. The data gathered from this experiment has provided the first DIS data in the high $x$ region. The DIS data on ${ }^{3} \mathrm{H}$ produced the first-ever measurement of the EMC effect for ${ }^{3} \mathrm{H}$ covering a large range of $x$ from 0.2 to 0.8 .

The goal of my research is to use the data from the MARATHON experiment to determine the EMC effect for both of the $\mathrm{A}=3$ systems. The following chapters will describe how I retrieve my EMC results. I will discuss the process of extracted the $\mathrm{A}=3 \mathrm{EMC}$ effect through chapters focusing on the production and detection of electrons, the analysis steps to count electrons, the method of measuring the cross section, and extracting the EMC effect. 


\section{Chapter 3}

\section{Experimental Setup}

\subsection{Thomas Jefferson Lab}

Thomas Jefferson National Accelerator Facility (JLab) in Newport News, Virginia hosted the MARATHON experiment in the Fall of 2017 and Spring of 2018. JLab uses support

from the U.S. Department of Energy (DOE) and the state of Virgina to complete the lab's mission of delivering productive research by exploring the atomic nucleus and its fundamental constituents, including precise tests of their interactions. Along with applying an advanced particle accelerator, particle detectors and other technologies to develop new basic research capabilities and to address the challenges of a modern society.

\subsubsection{CEBAF}

The Continuous Electron Beam Accelerator Facility (CEBAF) was recently upgraded to a $12 \mathrm{GeV}$ accelerator. The upgrade allows the accelerator to deliver a $11 \mathrm{GeV}$ beam of continuous electrons of up to $200 \mu \mathrm{A}$ of current to three experimental halls (A,B,C) and 12 $\mathrm{GeV}$ to the recently constructed hall D.

\subsubsection{Injector}

CEBAF uses a micro-pulsed structure from the photo-electron gun to produce electrons in an efficient manner. This micro-pulsed structure is used to prolong the lifetime of the 
photocathode. The delivery of unique current and energy to all four halls requires the micro-pulses to have a $250 \mathrm{MHz}$ or $500 \mathrm{MHz}$ structure and four individually tuned lasers. Frequencies of $250 \mathrm{MHz}$ and $500 \mathrm{MHz}$ are chosen because these are sub-harmonics of the fundamental accelerator operating frequency of $1500 \mathrm{MHz}$ [61].

Electrons are liberated when laser light shines on a gallium arsenide photocathode. A laser pulse excites electrons from the photocathode via the photoelectric effect. These electrons are ejected from the gallium arsenide wafer when the electrons are excited out of the valence band into the conduction band. Gallium arsenide was chosen because the energy level of the conduction band for this photocathode sits above the energy of an electron vacuum. Electrons in the conduction band escape from the material and accelerate away from the wafer due to high negative potential on the photocathode wafer [68].

Electrons that escape the photocathode wafer are accelerated into the injector beam line by the electron gun. A drawing of the injector beam line is shown in figure 3.1. Slits in the rotating chopper allow for regulation of the currents sent to the four experimental halls by reducing the number of electrons allowed through the chopper. Testing and calibration of the four beams are done throughout the injector beam lines via the Faraday cups and spectrometers located at different spots in the beam line. The polarized gun can supply electrons with up to $80 \%$ polarization and the polarization direction can be controlled by a Wien filter. The level of polarization is ensured through measurements from a $5 \mathrm{MeV}$ Mott polarimeter[4]. The injector accelerates the electrons up to $123 \mathrm{MeV}$ before allowing them into the north linear accelerator (LINAC) [57, 61, 74].

\subsubsection{Accelerator}

Electrons travel through two LINACs and two bending arcs per complete pass of the accelerator. The two LINACs are approximately a quarter-mile long and are thirty feet

underground. The beam lines are kept under vacuum between $10^{-6}$ and $10^{-11}$ torr to provide an efficient medium for transfer. Electrons traveling to Halls A, B, and C complete 


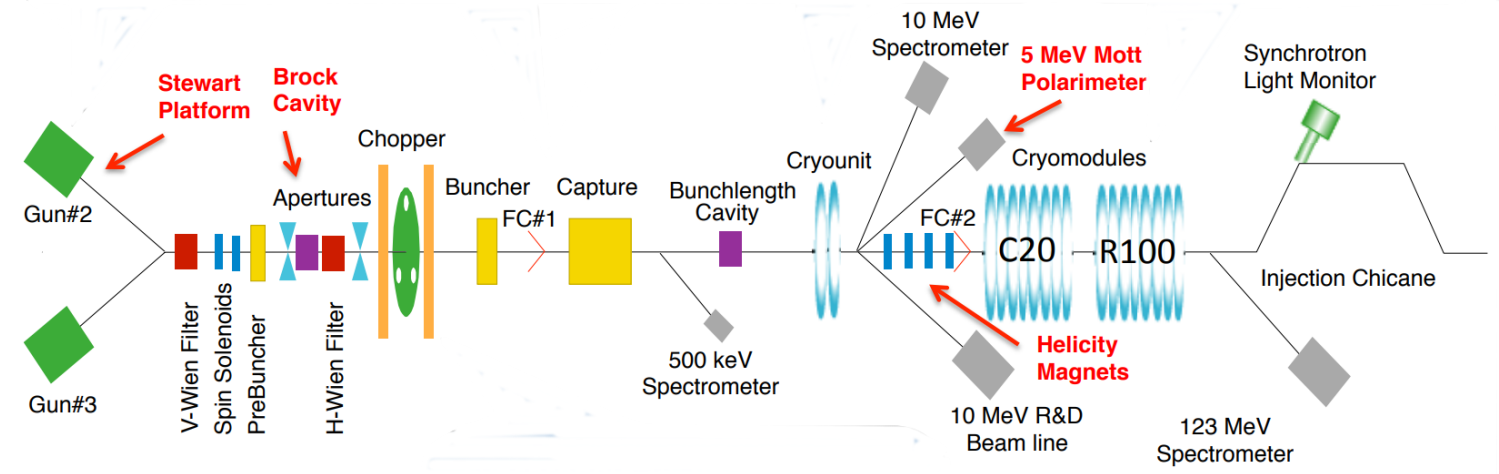

Figure 3.1: Drawing of the Injector layout.

a maximum of four and a half revolutions around the accelerator. These particles receive up to $2.2 \mathrm{GeV}$ in energy for each cycle through the accelerator.

The radio frequency $(\mathrm{RF})$ cavities in each LINAC use an oscillating electromagnetic field to supply a force to accelerate the passing electrons. These Niobium RF cavities are cooled to 2 $\mathrm{K}$ to create conditions that allow the cavities to be superconducting [4]. The superconducting RF (SRF) cavities provided a negatively charge field behind the electrons and positively charged field in front to accelerate the electrons through a set of cavities inside a cryomodule. A central helium liquefier circulates up to 17000 gallons of chilled liquid helium to control the temperature of the cryomodules. A dedicated $5 \mathrm{~kW}$ klystron provides a $1500 \mathrm{MHz} \mathrm{RF}$ driving signal for each cryomodules.

The electron beam exiting the north LINAC enters the east arc. The east and west arcs contain large dipole and quadrupole magnets to steer and focus the beam as it accelerates back to the other LINAC. After electrons exit the south LINAC, they either continue on around the accelerator for another pass to increase in energy, or a RF separator projects the electron beam into the proper experiment hall [61]. Figure 3.2 shows a general layout of CEBAF, including the experiment halls. Energy loss, beam position, and beam charge monitors lie throughout the beam line, and are used to ensure high quality beam delivery to the experimental halls. The accelerator staff in the machine control center (MCC) and the 


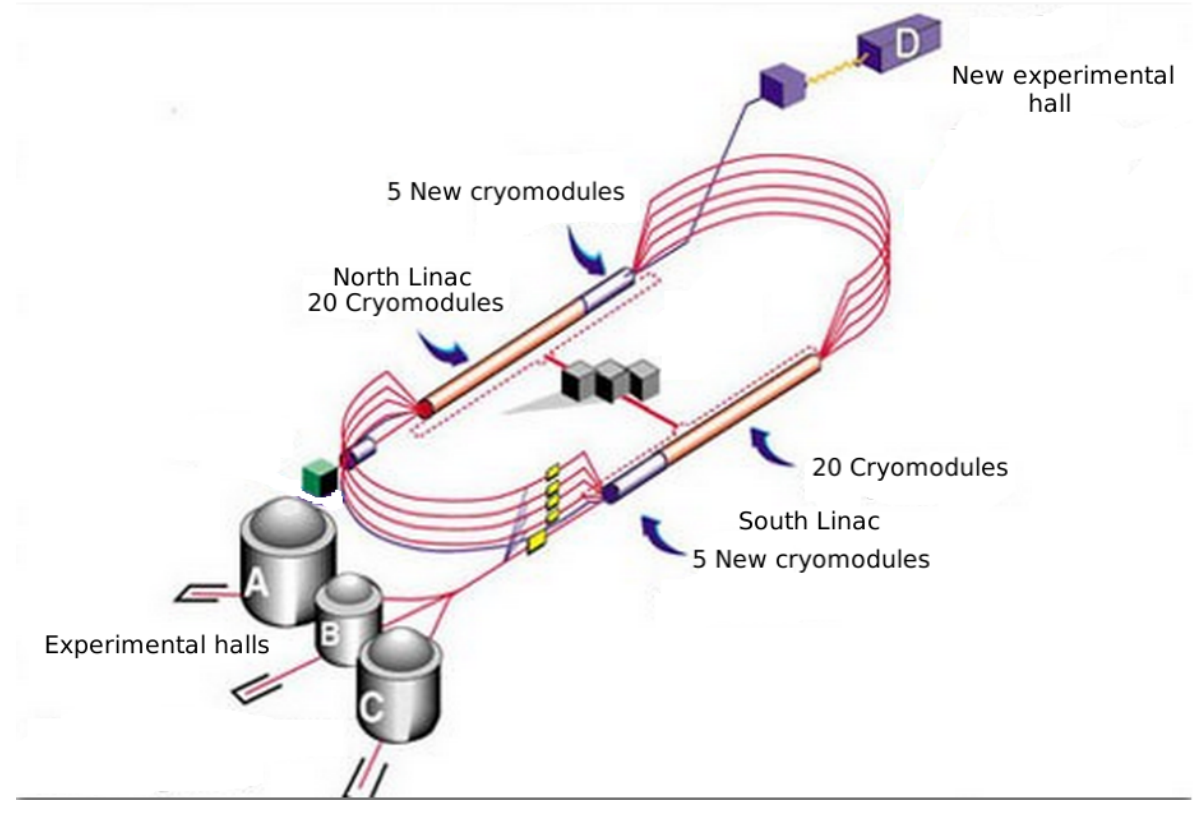

Figure 3.2: Schematic Layout of CEBAF.

experimentalist in the experimental halls collaborate together to provide an atmosphere for safe and efficient scientific discovery.

\subsection{Hall A Beam Line}

The experimental Hall A and the scientific equipment used were designed for detailed investigations of electro and photo-induced reactions. Two high resolution spectrometers in Hall A use the inclusive (e,el) and exclusive (e,e/ p) reactions to gain a greater understanding of the structure of the nucleus. Completing detailed studies with high resolution and extreme accuracy requires knowing the beam position, size, energy, and current when the beam strikes the target. The instrumentation used in the precise measurement of these quantities in Hall A are shown in figure 3.3 [4]. The information provided by these detectors originate through small changes in current and voltage sent through the electronics. Detector calibrations turn the signals from these detectors into useful information. 


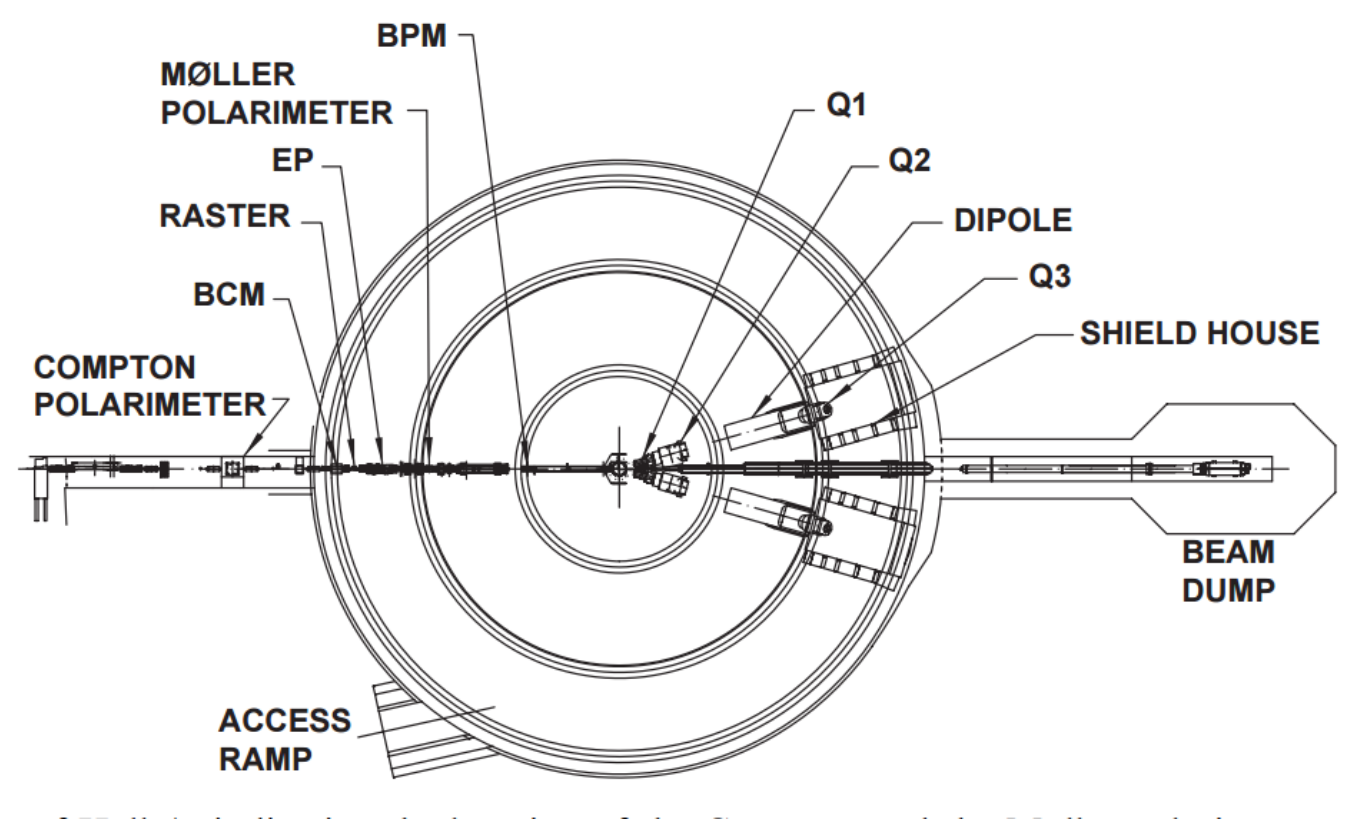

Figure 3.3: A schematic layout of the beam line in Hall. [4]

\subsubsection{Beam Position Monitors}

A pair of Beam Position Monitors (BPM)s measure the relative beam position without affecting the beam. The two Hall A BPMs are located at $7.524 \mathrm{~m}$ and $1.286 \mathrm{~m}$ away from the target. Using the standard difference-over-sum technique, the relative beam position is determined with an accuracy of $100 \mu \mathrm{m}$ with a beam current of at least $1 \mu \mathrm{A}$ [4]. The BPMs' positional data is recorded in two ways. Every second of beam time, the beam position, averaged over 0.3 seconds, is logged into the Experimental Physics and Industrial Control System (EPICS) database. The BPMs also transmit data event-by-event to the CEBAF online Data Acquisition system (CODA).

The main beam line components of the BPMs consist of four open-ended antennas. Figure 3.4 shows a side view of a BPM chamber and figure 3.5 shows the layout of the four antennas as you look down the beam line. The antennas are titled $u_{+}, u_{-}$and $v_{+}, v_{-}$. The antennas receive an induced signal as electrons pass to determine the beam position in the $\mathrm{u}$ and $\mathrm{v}$ directions. The BPMs send a DC offset to the DAQ. This DC offset is turned into a positional measurement via an ADC calibrated signal. The position of one axis is determined through 
the difference over sum method:

$$
u=\frac{u_{+}-u_{-}}{u_{+}+u_{-}} .
$$

The beam position in the frame of the $\mathrm{u}$ and $\mathrm{v}$ antennas are calculated by the taking the difference over the sum of the two wires in the $u$ and $v$ directions. The accuracy of the BPMs requires an absolute measurement of the electron beam's position to calibrate the BPMs $[77,86]$.

Figure 3.6 shows an image of the harps used for BPM calibration. Each harp is located immediately after the BPM on the beam line. The harp forks are aligned perpendicular to the beam line to allow the harps to move in and out of the beam line. A wire that traverses between the fork tines at three different angles in respect to the harp detects electrons passing through the beam line. The two sloped sections of the wire are angled at $45^{\circ}$ relative to the harp frame. As the harp fork moves into the beam, the wires receive a signal as the beam interacts with the wires. The signal strength from the harp wire represents how close the wire is to the beam. A peak in the signal demonstrates the location of the beam in respect with the corresponding wire. The two sloped wires are used together to determine the vertical position of the beam. The vertical wire is used to determine the horizontal position of the beam $[77,86]$. The harps are not used during production phases due to their intrusive nature caused by the interaction of the beam with the harp wire.

The location of the wires on the harp frame and the position of the harp fork were used to calculate the absolute beam position. Figure 3.7 shows an example of five positions used to calculate the BPM calibration coefficients and the BPM position reading before calibration. The light gray symbols are the beam locations from harp measurements, while the colorful markings are measurements from the BPMs. This method of using beam positions at the nominal center and surrounding the center is called a bull's eye scan. The harp scan results are substituted into equation 3.2 for the $\mathrm{X}$ and $\mathrm{Y}$ positions. Using all five points and an $R^{2}$ regression technique, the coefficients can be determined with great accuracy. Figure 3.8 shows the comparison between harp position and BPM after calibration. These highly 


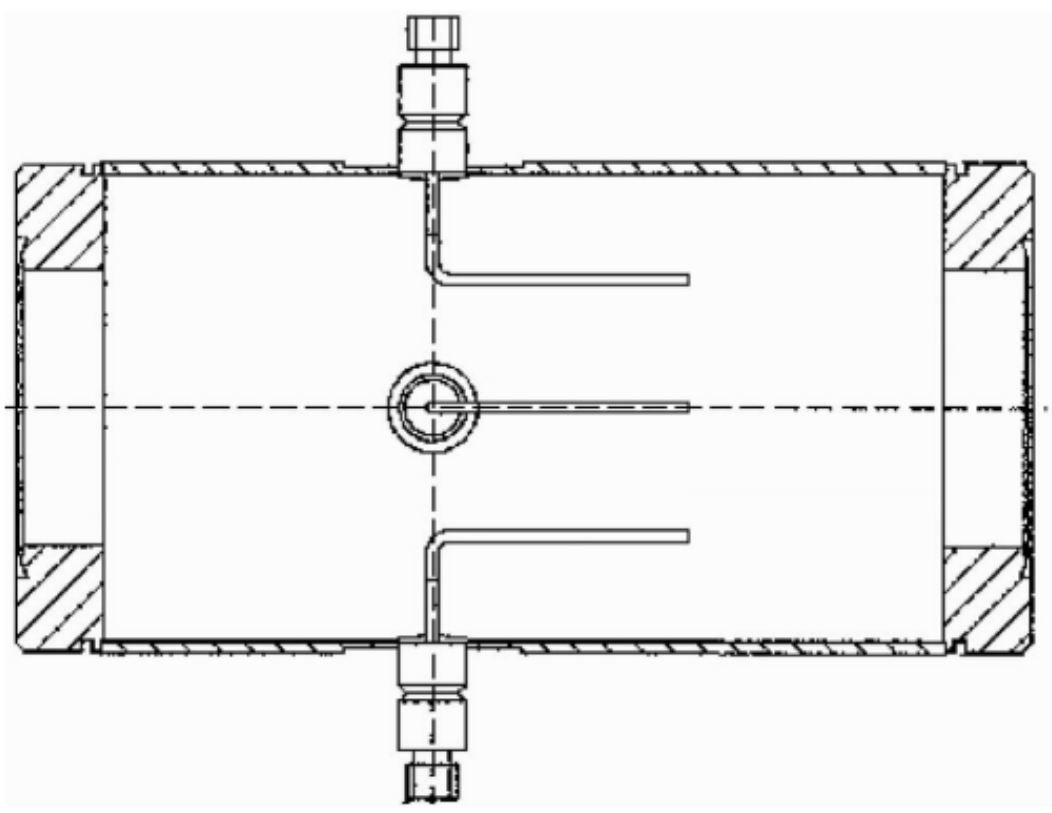

Figure 3.4: BPM design diagram, from JLab instrumentation group. Beam direction is from left to right [86].

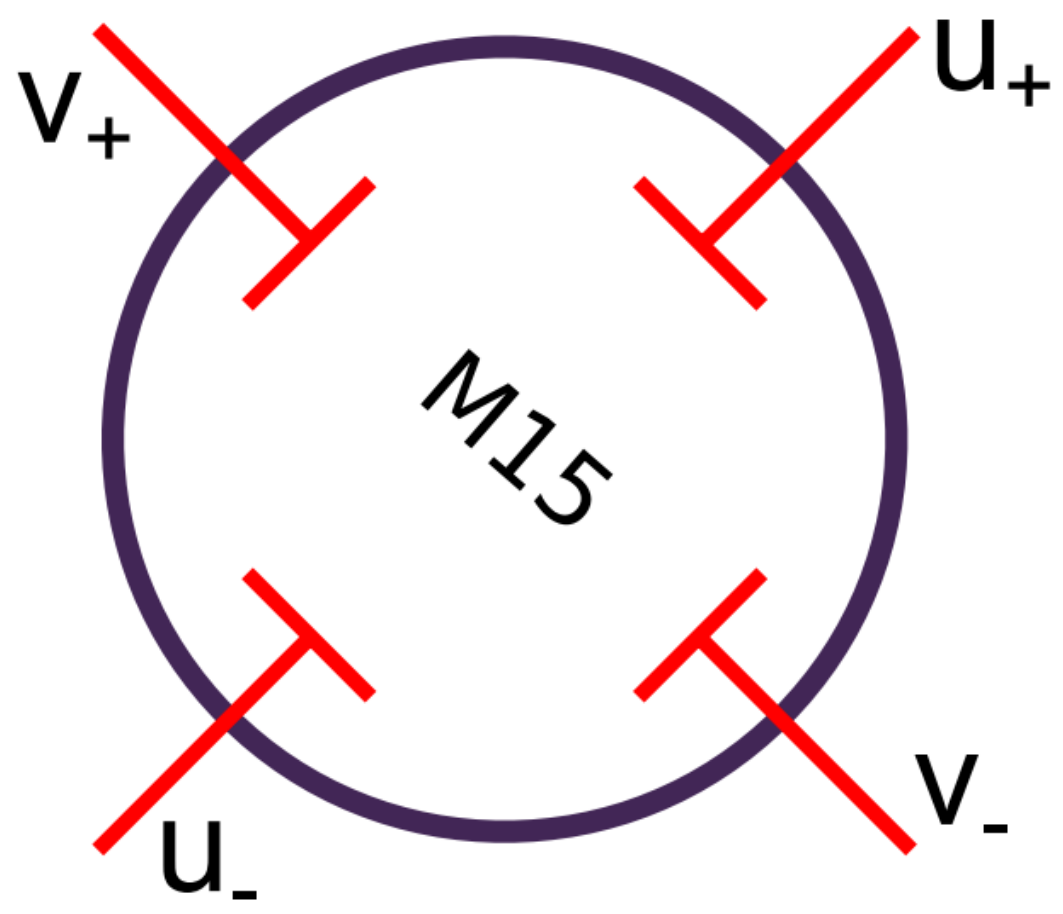

Figure 3.5: BPM design diagram, looking down the beam line[86]. The diameter of the beam line is restricted to $63.5 \mathrm{~mm}$ [4]. 
Figure 3.6: A schematic layout of a harp fork [86]

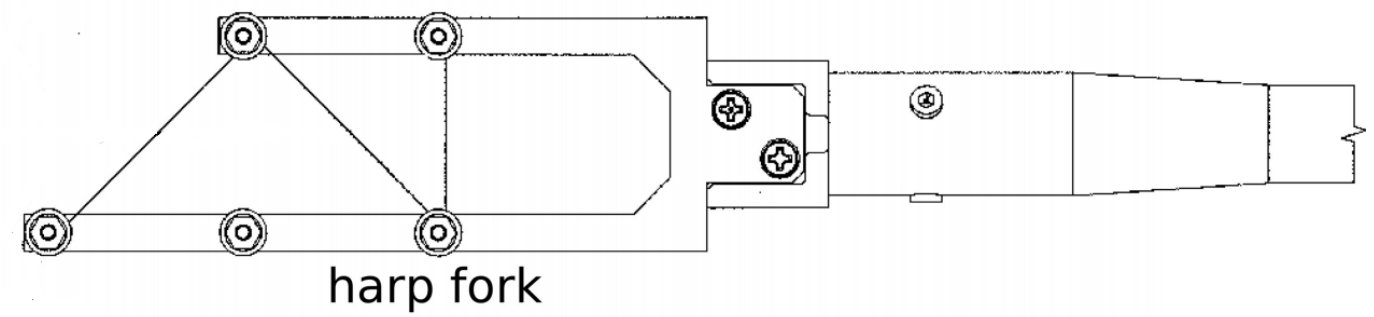

accurate BPMs were crucial in reducing systematic error in the final results obtained from this experiment.

$$
\left(\begin{array}{c}
X_{\text {position }} \\
Y_{\text {position }}
\end{array}\right)=\left(\begin{array}{ll}
C(0,0) & C(0,1) \\
C(0,0) & C(0,1)
\end{array}\right) \times\left(\begin{array}{c}
X_{B P M} \\
Y_{B P M}
\end{array}\right)+\left(\begin{array}{c}
X_{\text {offset }} \\
Y_{\text {offset }}
\end{array}\right)
$$

\subsubsection{Raster}

Damage to a target system from intense beam can cause extreme fluctuations in the target's temperature and density. A raster was used to counteract the damage caused by a focused beam. A raster uses two magnetic fields produced by two dipoles to spread the electron beam out. This produces a large rectangle interaction area on the front face of the target container. A triangle wave of $25 \mathrm{kHz}$ controls the coils of the dipole magnets. The raster system begins $\approx 17$ meters before the target chamber [86]. The raster system's relative position can be seen in figure 3.3. Safety constraints administrated by the target group at JLab limited the minimum size of the raster spot for the MARATHON experiment to two millimeters by two millimeters. The $2 \times 2 \mathrm{~mm}$ minimum limit for the raster size was installed as a preventative safety measure to eliminate concerns of the ${ }^{3} \mathrm{H}$ gas breaking containment through damage to the target cell entrance window.

The Hall A raster system consists of four dipoles. Two dipoles produce magnetic fields in the horizontal direction of the lab frame and two in the vertical. The upstream raster and downstream raster include one vertical and one horizontal dipole. The relative change 


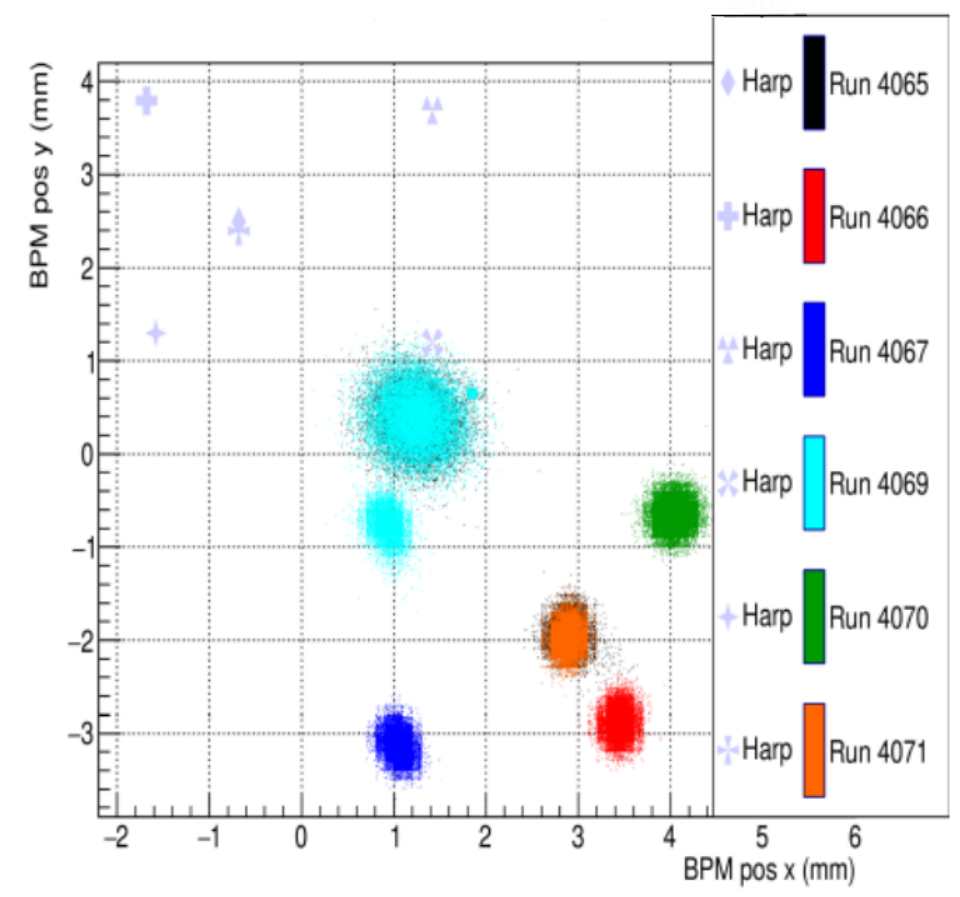

Figure 3.7: The $\mathrm{X}$ and $\mathrm{Y}$ position comparison for harp to BPM for a bulls eye scan before BPM calibration.

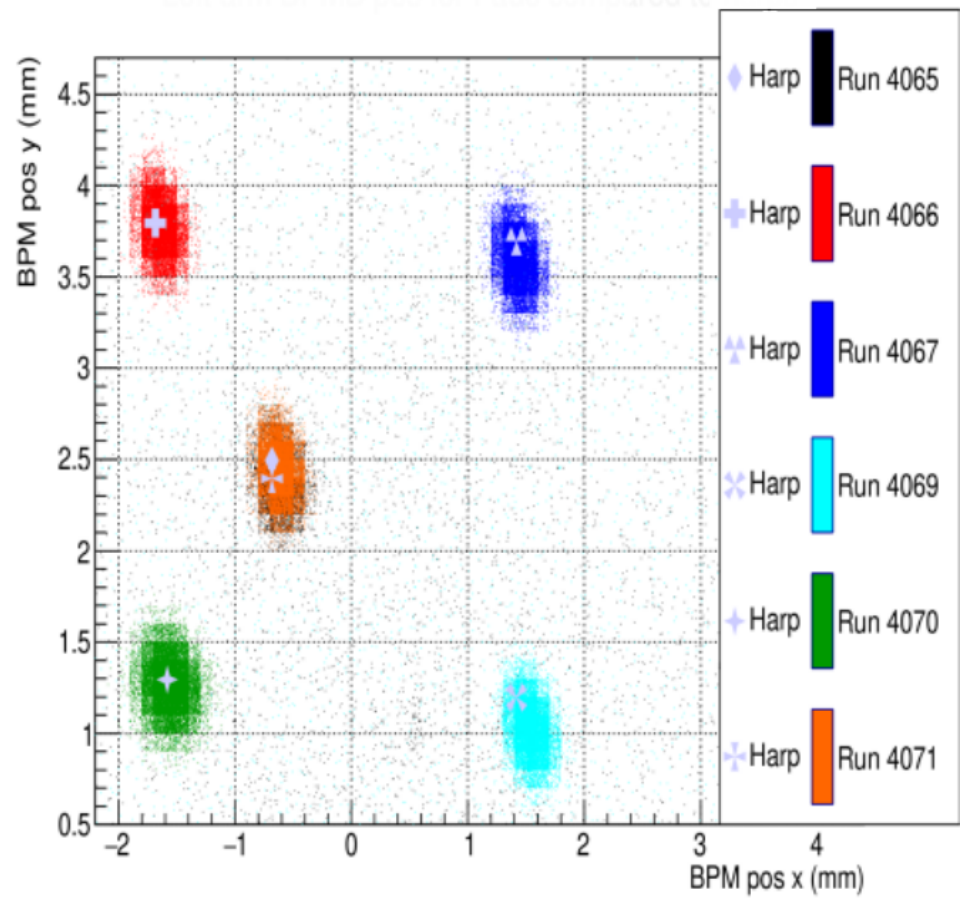

Figure 3.8: The X and Y position comparison for harp to BPM for a bulls eye scan after BPM calibration. 
in position of the incoming electrons are controlled by the current supplied to the dipoles. This current that drives the dipoles is recorded by an ADC. In order to obtain the change in beam position due to the raster, a calibration between the raster current and measured beam position were obtained.

The raster calibration is done by creating a line that maps the raster current measured by ADC channels to a position. This calibration process is done to extract beam positions at the locations of both BPMs and the target center along the beam line. Calibrating the raster requires two calibration procedures. The first process was to determine the size of the rastered beam spread. In order to accurately determine the width and height of the beam spread due to the raster, a carbon foil with a hole of a diameter of $2 \mathrm{~mm}$ was used. Events will only scatter from outside of the hole. Plotting the $\mathrm{x}$ and $\mathrm{y}$ raster current of the rastered beam will show the hole through a vacancy of events. The fit of the carbon hole gives the width of the raster, the slope of the linear mapping term. In figure 3.9, the raster current in $\mathrm{x}$ and $\mathrm{y}$ directions are fitted using this radial sigmoid. Once the slope of the linear calibration is determined, the offsets can be found. This is discovered by using the calibrated BPM mean positions for a phase of rastered beam. The mean positions for both BPMA and BPMB produce a track from the BPMs to the target. This projection provides a mean location of the beam at the target. Using equation 3.3, the offsets also known as the intercepts are solved for using the slope $\left(m_{x}, m_{y}\right)$, the raster mean current value $\left(R_{x}, R_{y}\right)$, and the mean BPM position $(x, y)$ [49].

$$
\left(\begin{array}{l}
x \\
y
\end{array}\right)=\left(\begin{array}{l}
R_{x} \\
R_{y}
\end{array}\right) \times\left(\begin{array}{cc}
m_{x} & 0 \\
0 & m_{y}
\end{array}\right)+\left(\begin{array}{c}
O_{x} \\
O_{y}
\end{array}\right)
$$

\subsubsection{Beam Energy}

The electron beam energy is located in many of the equations used in an electron scattering experiment. This can cause a noticeable increase in systematic error if the beam energy measurement is not made precisely. In Hall A, the beam energy was measured by using the (e,e/p) method. On the beam line, 17 meters upstream from the target an ep scattering chamber is located. MMC directs the beam into the ep scattering chamber containing a 


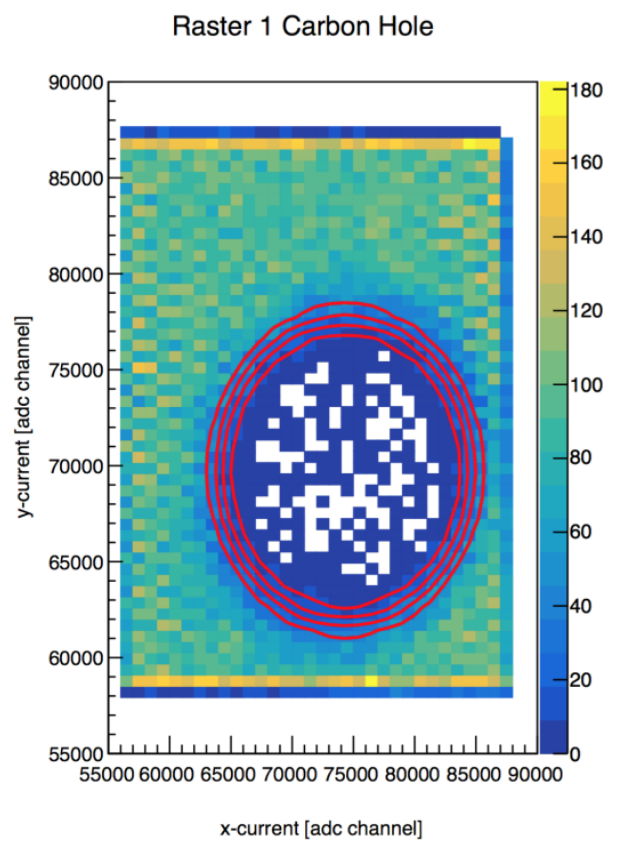

Figure 3.9: The $\mathrm{X}$ and $\mathrm{Y}$ current of the raster with a carbon hole. The size of the carbon hole is fit with a radial sigmoid[49].

rotating 10-30 $\mu \mathrm{m}$ thick tape of $\mathrm{CH}_{2}$. The scattering angle of the electron and the recoil angle of the proton are used to determine the beam energy using equation 3.4. Where $M_{p}$ is the mass of the proton and $\theta_{p}, \theta_{e}$ are the scattered angle of the proton, electron respectively.

$$
E=M p \frac{\cos \theta_{e}+\frac{\sin \theta_{e}}{\tan \theta_{p}}-1}{1-\cos \theta_{e}}
$$

The beam energy was also measured using the arc measurement method [40]. This method uses changes is beam position and precise measurements of the magnetic fields around the beam line to determine the energy of the electron beam. The angle at which the electrons bend through the magnetic field relates to the momentum of the electrons,

$$
p=k \frac{\int \vec{B} \cdot d \vec{l}}{\theta}
$$

In equation $3.5, \mathrm{p}$ is the momentum of the electrons, $\theta$ is the bend angle, and $\vec{B}$ is the magnetic field the electrons experience. Then using the momentum of the electron, the energy of the beam can be extracted. The error on the beam energy measurement is $\delta$ 


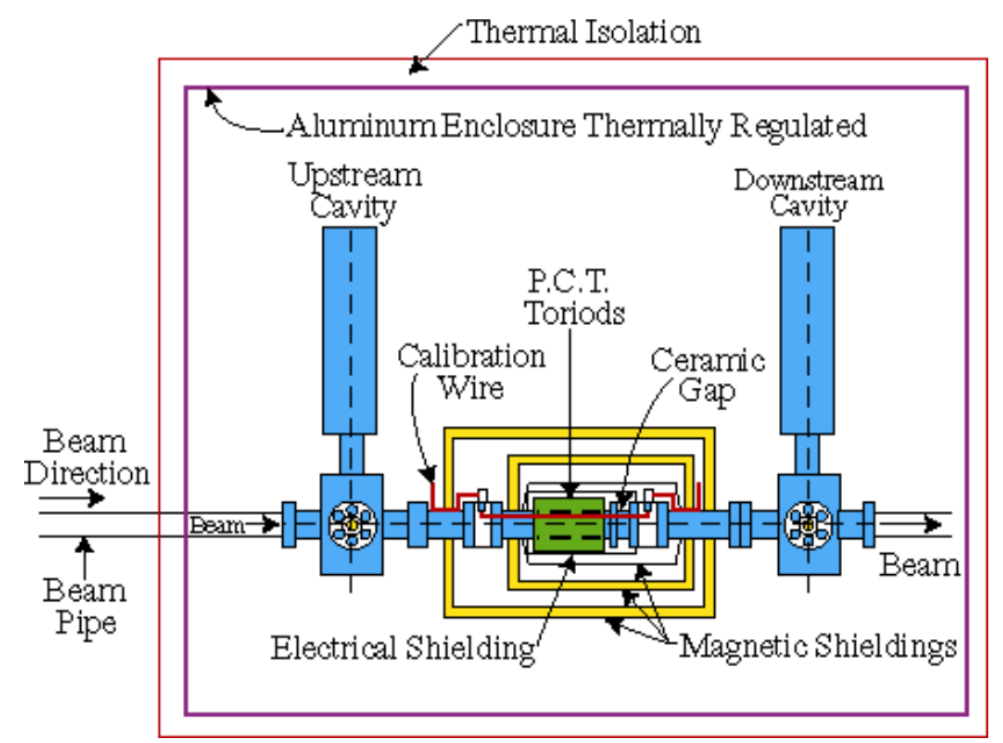

Figure 3.10: Hall A Current Monitor components [35].

$\mathrm{E} / \mathrm{E} \approx 2 \times 10^{-4}[83,40]$. The MARATHON experiment used both methods to accurately determine the electron beam energy.

\subsubsection{Beam Current Monitors}

The main process of measuring the scattering yield for a calculation of a cross section looks at finding the ratio of the number of electrons scattered to the number of electrons sent. In order to accurately determine the number of electrons sent to scatter with our target system, Hall A use a set of non-invasive beam current monitors (BCMs). The Hall A BCMs have an absolute accuracy of 0.2 percent as long as the current is between 1 and $180 \mu \mathrm{A}$. The BCMs used in Hall A consist of three main components: a Parametric Current Transformer (PCT) and two pill box cavities. Figure 3.10 shows the components in the Hall A BCM. The BCM produces an RF signal that is proportional to the beam current. A $10 \mathrm{kHz}$ down converter, RMS-to-DC converter, voltage-to-Frequency converter, and a scaler convert the are used to inject the current signal into the Hall A DAQ. Proportionality constants are determined in the calibration process to correctly integrate the charge for a given amount of beam current[35]. 


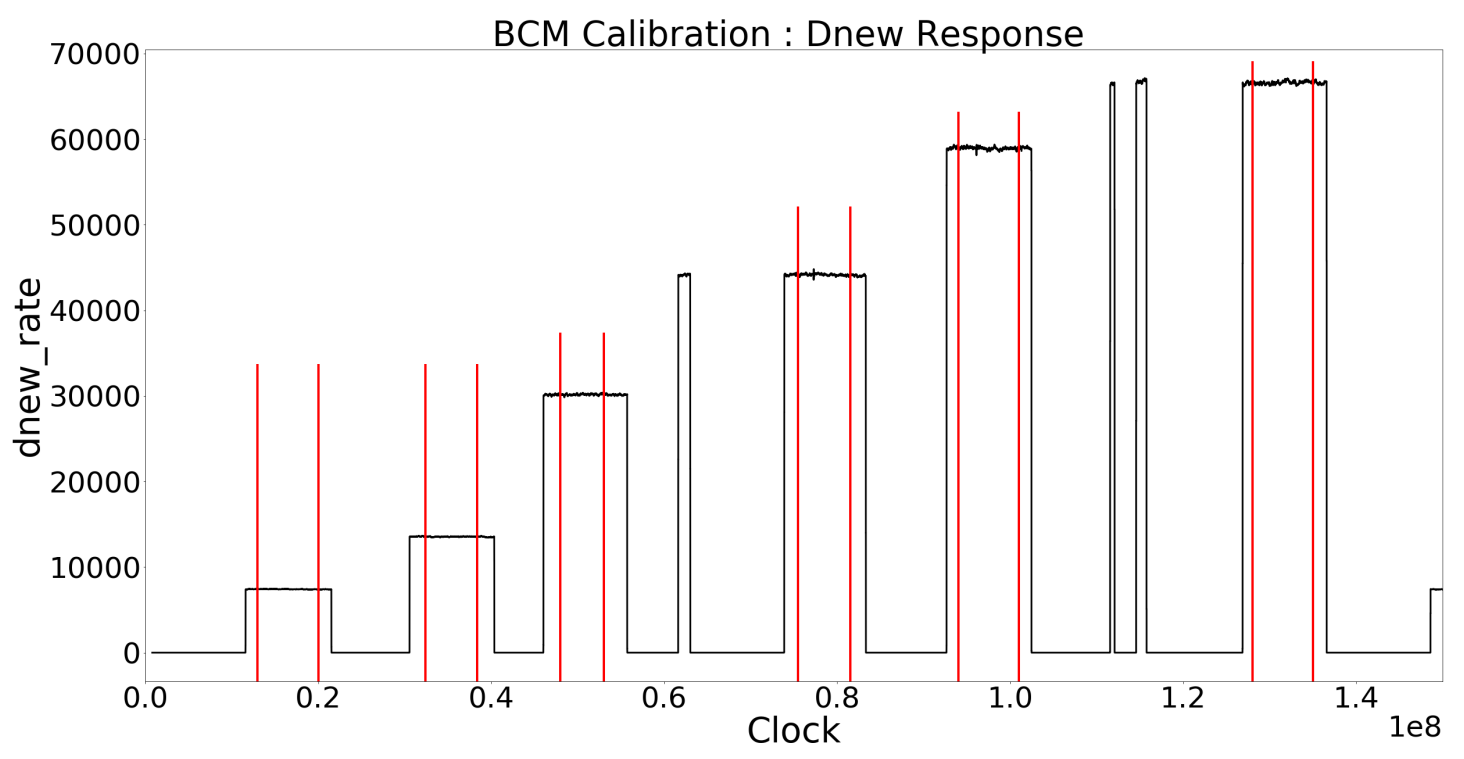

Figure 3.11: BCM calibration, changing the current, observing the rate for dnew, a downstream digital BCM [73].

The process of calibrating the $\mathrm{BCM}$ converts the frequency received from the BCMs to an amount of current in $\mu \mathrm{A}$. In order to calibrate the BCMs in Hall A, a separate intrusive calibration of an unser must be done. The unser is calibrated by inserting a known current through a wire inside the beam pipe. The calibration of the unser is known to drift over time, which makes the unser unfeasible to use as the main source of charge calculation. Once the unser is calibrated, the BCM calibration procedure can be completed. The BCM calibration requires the delivery of the electron beam with unique procedure. This process consists of oscillating the beam on and off status while increasing the current. Figure 3.11 shows the process of alternating current on and current off at different magnitudes of current. This stepping up procedure provides an adequate number of data points to complete a linear fit of the BCM frequency verses the calibrated unser current. The linear fit parameters supply a multiplicative gain and an additive offset for the calibration of the BCMs. Figure 3.12 shows a linear fit that provides gain and offset calibration constants for the BCM used in the calculation of charge. 


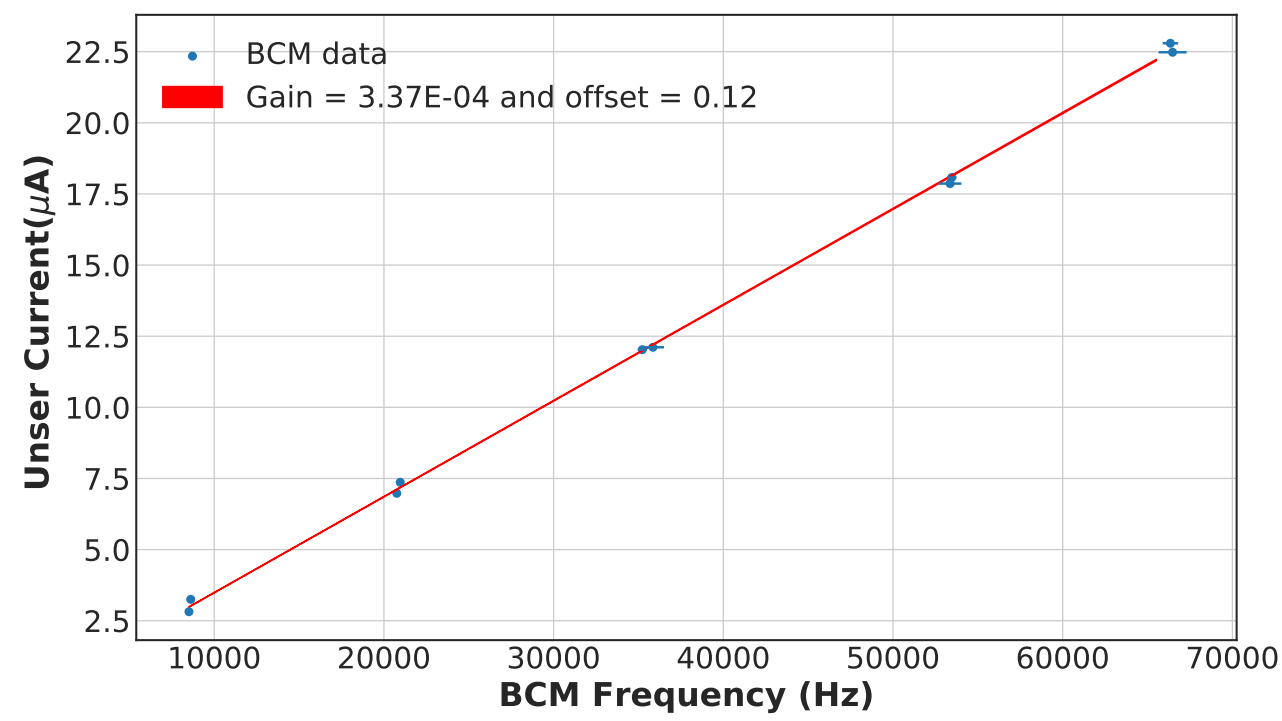

Figure 3.12: The relationship between unser current and BCM frequency for BCM calibration [73].

\section{$3.3 \quad$ Target}

The ${ }^{3} \mathrm{H}$ run group of experiments, including MARATHON, used the newly designed Hall A ${ }^{3} \mathrm{H}$ Target (HATT) system. The HATT target chamber was repurposed from a previously used cryogenic target chamber to reduce the financial cost of designing a new target chamber. The refurbishing of the cryogenic target chamber consisted of adding in new safety features to prevent and mitigate a ${ }^{3} \mathrm{H}$ leak. A 4 inch long collimator with an inner diameter of 0.4 inch was added inside of the target chamber upstream of the target ladder to prevent the beam from striking the thin side wall of the aluminum cell. In case of a ${ }^{3} \mathrm{H}$ leak in the target chamber, an exhaust system was installed to control the amount of ${ }^{3} \mathrm{H}$ exposed to the Hall.[66] Figure 3.13 shows the HATT system with the target ladder in the home position and the scattering windows removed. A picture of the HATT ladder installed in the HATT system is shown if figure 3.13. The ladder contains both gaseous cells and solid targets. The MARATHON experiment had five gas cells. The top four of the gas cells were filled with ${ }^{3} \mathrm{H},{ }^{2} \mathrm{H}, \mathrm{H}$, and ${ }^{3} \mathrm{He}$, from top to bottom. Due to safety restricts the ${ }^{3} \mathrm{H}$ cell was not installed until the HATT system could be closed. The bottom most cell was left empty, to complete end cap subtraction. The lower half of the target ladder contains the solid targets 


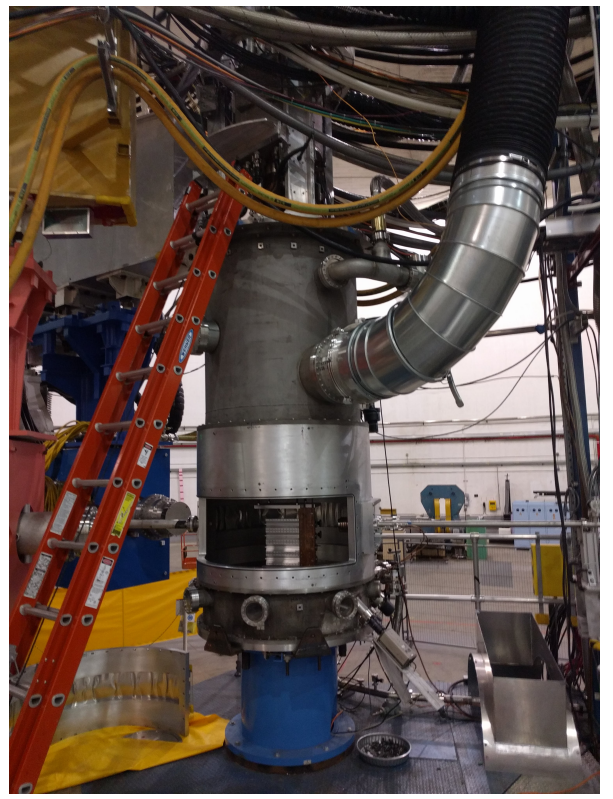

(a) A image of the HATT. [53]

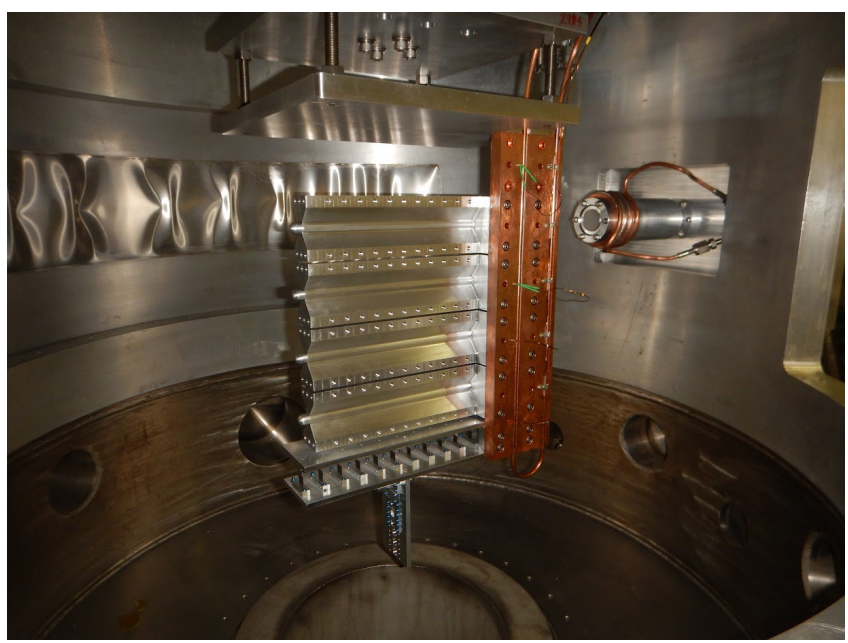

(b) Image of the Hall A ${ }^{3} \mathrm{H}$ Target Ladder. [53]

Figure 3.13: Target Images

used during the MARATHON experiment. The target cells are mounted to a heat sink with flowing cryogenics for temperature control of the target cells. Listed from top to bottom, the solid targets used were a pair of thick aluminum foils, carbon multifoil, single carbon foil, and a carbon foil with a $2 \mathrm{~mm}$ diameter hole. The thick Al foils were used to aid the target window background subtraction. The multifoil target also know as the optics target was used to calibrate the z-axis reconstruction of the optics matrix. The single carbon foil and carbon hole were used to calibrate the BPMs and raster and to determine the off set of the central line of the detector.

\subsection{High Resolution Spectrometers}

Electrons that successfully scatter from the target may end up in either of the two HRSs (High Resolution Spectrometers). The HRSs were designed to detect charged particles with a high degree of precision. In order to achieve a high level of resolution in momentum and angle, the design of the HRSs consist of a magnet configuration of QQ $D_{n} \mathrm{Q}$ (quadrupole, quadrupole, dipole, and quadrupole). The vertical bending dipole provides the field required 


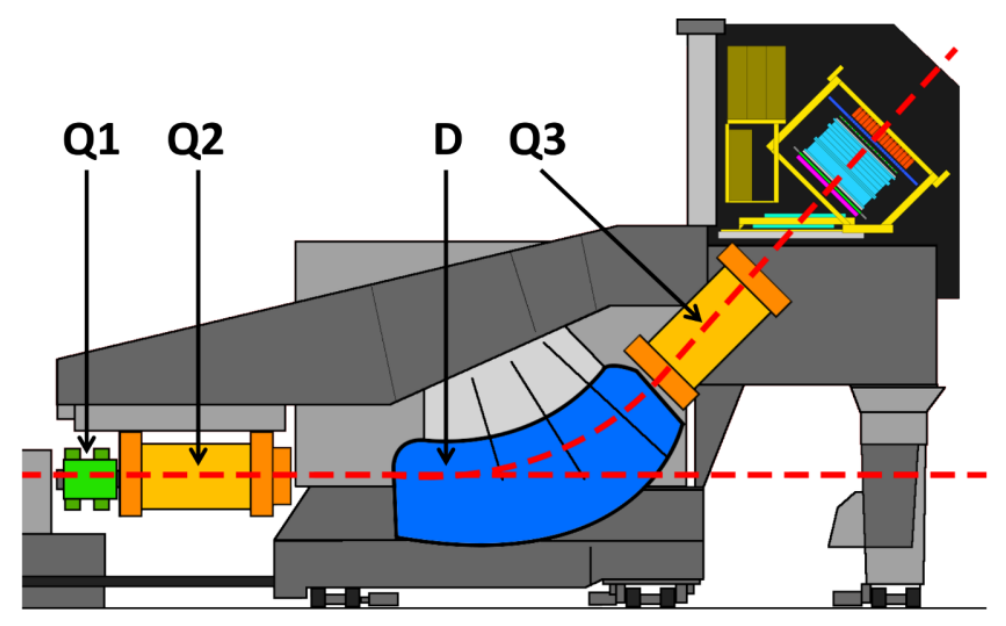

Figure 3.14: A side view of a HRS [4].

to transport the scattered particles through the $45^{\circ}$ bending angle to the detector hut. A drawing of an HRS can be seen in figure 3.14. The first quadrupole (Q1) focuses the incoming electrons in the vertical plane. The following two quadrupoles (Q2 and Q3) provide transverse focusing. This optical design allows the use of extended gas targets with no substantial loss in solid angle[4].

The spectrometer's design allows for the performance of various functions, which include: triggering the data acquisition system (DAQ) when certain requirements are met, gathering the position and direction of individual particles to reconstruct a track, provide precise timing information for time of flight calculations, and identify many different particle types that pass through the detector system. In order for both the Left HRS (LHRS) and Right HRS (RHRS) to complete the required task, they contain a collection of different detectors. The HRSs use drift chambers, scintillators, Cherenkov detectors, and shower calorimeters. Both the Left and Right HRSs contain two planes of scintillators to function as the main trigger for the detector package. The vertical drift chambers (VDC) that lay at the front of the detector in conjunction with the Shower that lies in the back of the detector provide information for reconstructing the particle tracks and precise timing. Particles are identified by the Cherenkov, shower calorimeters, and pion rejectors that live in the left or right HRS. 


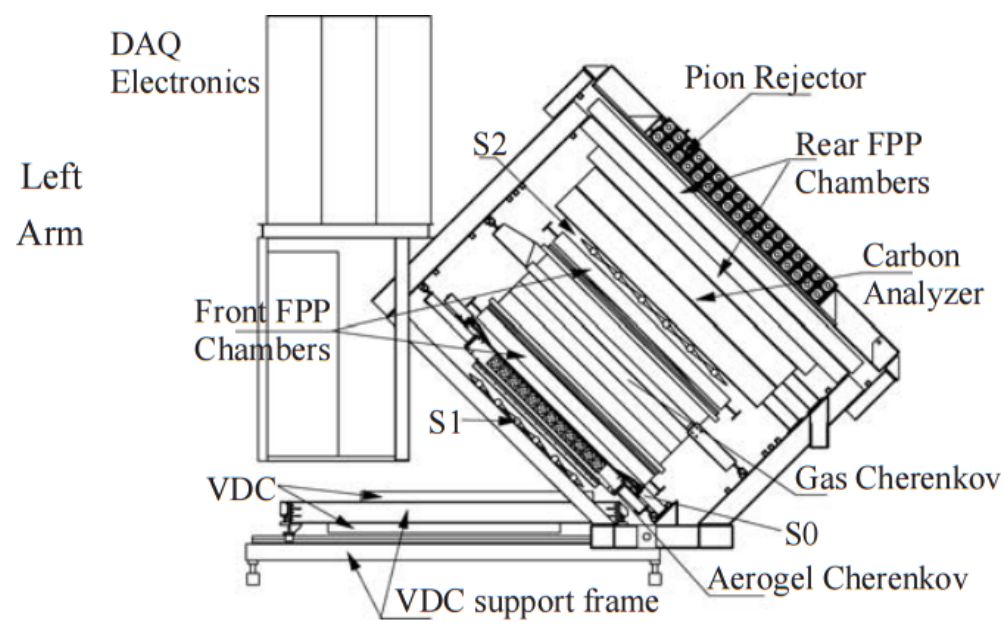

Figure 3.15: A view of both the left (top) and right (bottom) detector stacks inside the left and right HRS [4].

The layout of the individual detectors that make up the left and right detector package are shown in figure 3.15 [4].

\subsubsection{Vertical Drift Chambers}

Each of the spectrometers housed in Hall A contains two vertical drift chambers (VDC). Each VDC incorporates two planes of crossing sense wires. Shown in figure 3.16, the two planes of the VDC lie a distance of $0.335 \mathrm{~m}$ apart [39]. The lower plane of the VDC is positioned at the approximate focal plane of the HRS and lies in the horizontal plane of the Hall A coordinate system. The sense wires located in the VDCs cross orthogonally. They are offset by $45^{\circ}$ with respect to the dispersive and non-dispersive directions. Each plane of the VDC uses 368 sense wires, with $4.24 \mathrm{~mm}$ between each wire. The signals from these wires are transmitted to the electronics via a set of printed circuit boards that contain a 16-channel connector and twisted pair ribbon cables. These ribbon cables transmit the VDC signal to a set of common stop TDCs with 0.5 ns resolution [39]. The VDC sense wires are held at ground potential between two planes of high-voltage. Particles that enter the gas-filled VDC, collide with molecules of an argon (62\%) and ethane (38\%) mixture [4]. This collision causes the ionization of the gaseous mixture producing drift electrons. 


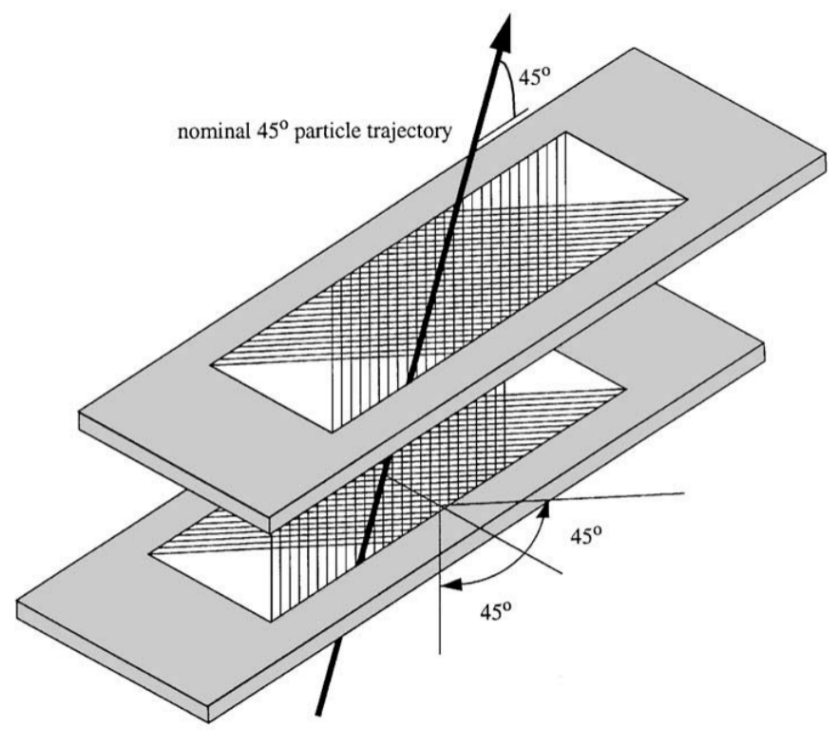

Figure 3.16: A sketch of the two VDC planes in the HRSs with a particle traveling through the detector at $45^{\circ}$.[39].

Particles that traverse the VDCs will travel through regions close to several sense wires. As the incident particle ionizes gas in each of these regions, the VDC sense wires pick up the corresponding signal from the drift electrons. The drift electrons will travel to the sense wires via the parallel electron field lines until the electrons get close to the sense wires. Once close to the sense wires, the electric field transitions to a radial field and the drift electrons then move to the sense wires.

The drift chamber's performance is constantly monitored throughout the experiment. The efficiency of an individual wire is determined by an algorithm that scans a plane for an event that fires a cluster of wires. A wire is determined to be efficient for that event if it fired along with its two nearest neighbors. This efficiency calculation is used during the online analysis to keep track of the performance of the VDCs and to assist in the maintenance of the HRSs throughout the experiment.

The VDC's main task during an electron counting experiment is to determine the track of the scattered electron. The track of the electron is used to ascertain the electron's scattering momentum and scattering angle. Due to the electron's relativistic nature the primary 

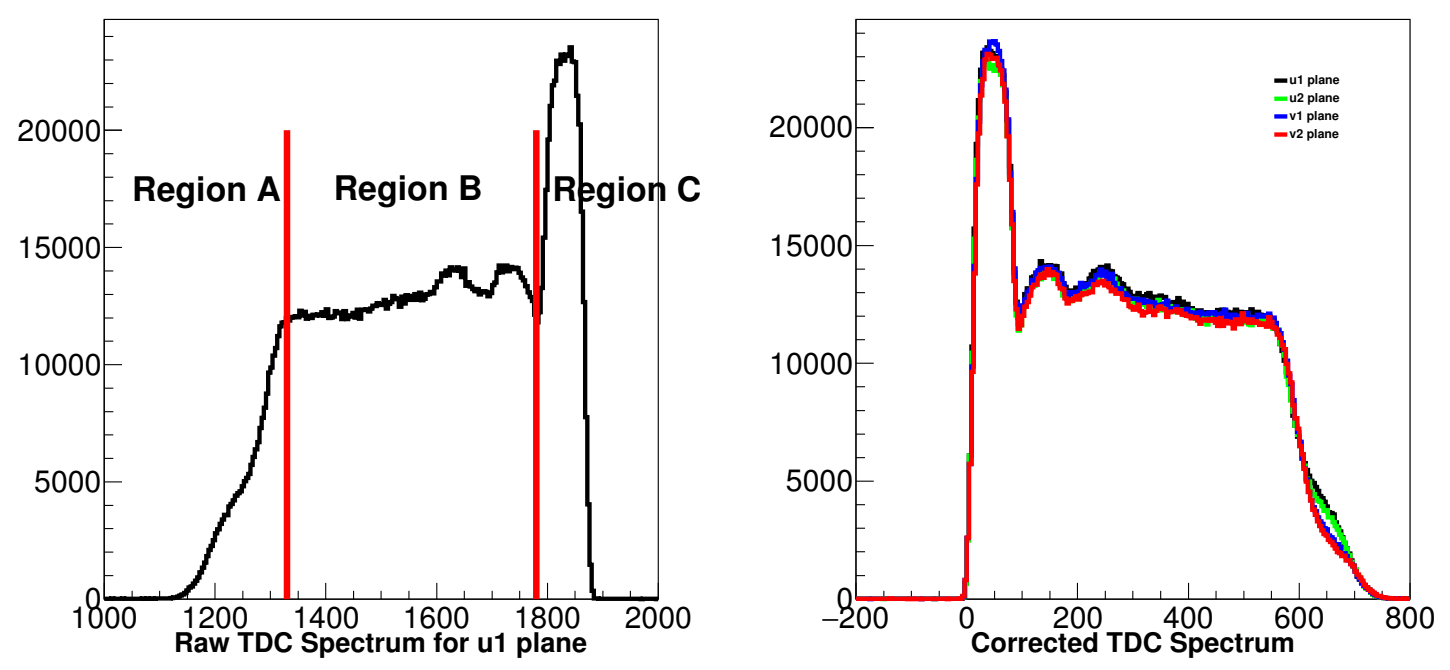

Figure 3.17: Histograms of VDC signals before (left) and after (right) calibration of t0[17].

ionization event for each wire region happens simultaneously compared to the resolution of the TDCs. The common stop TDCs used for the VDC signals record the amount of time from drift electron's signal in the sense wires to the stop signal formed by the trigger. This creates a high TDC signal for short drift distances. The raw TDC values recorded by the VDC include time associated with the signal but also the time required to form the trigger and time of flight for electrons between the VDCs and detectors used in the formation of a trigger. The calibration of the VDC removes these extra sources of time in the TDC signal. In order to calibrate the VDC raw signals, a reference time is determined for every wire on every plane. The sharp decrease on the outside of the peak in region $\mathrm{C}$ shown in figure 3.17 defines the references time $\left(t_{0}\right)$ of a VDC's TDC signal. Figure 3.17 shows histograms for before and after TDC calibration for the VDCs. The spectrum is divided into three regions.

- Region A: In this region, the point of primary ionization is far from the sense wire. As this distance increases, the chance of detecting the traversing particles by this wire decreases.

- Region B: The probability of sense wires detecting a primary ionization event in this region are uniform due to the uniform electric field though out the region. 
- Region C: The primary ionization position for these events is very near the sense wire and the electric field from this area is going to change to radial shape and the probability to detect a particle is going to increase in this area [17].

The time recorded from the TDCs is used to construct the location of an ionization event for each sense wire across the scattered electron's trajectory. The analyzing software will use these drift distance from the four VDC planes to find a track for the scattered electron.

\subsubsection{Scintillators}

A pair of scintillator planes form the primary triggering apparatus for the HRSs. The planes of scintillator S0 and S2 consist of a collection of plastic scintillating paddles with photo multiplier tubes (PMTs) attached to both ends of the paddle. S0 the first scintillator in the stack consists of one scintillating paddle in a vertical direction. S2, the second scintillator was built with 16 overlapping paddles with PMTs attached to both ends. As electrons enter the scintillating plastics, photons are emitted via the scintillating interaction. These photons are detected by the PMTs on either side of the scintillator bar. The passing of the electron can happen at positions at an unequal distances from the PMTs on a scintillator bar. These relative differences cause a distortion in the timing calculation in the time of flight (TOF) known as the time walk effect. The scintillators are used in the calculation of $\beta$, the relativistic $v$ to $c$ ratio. Beta is calculated using the TOF between the two scintillator planes and distance traveled between the points of interaction. Once calibrated, each plane has a time resolution of about $0.3 \mathrm{~ns}$. This high time resolution and quick response makes the scintillators the perfect detector to form the main trigger.

\subsubsection{Cherenkov}

After a particle passes through S0, it will enter the large gas chamber for the gas Cherenkov (GC). The GC is filled with $\mathrm{CO}_{2}$ with an index of refraction of 1.00041. This high index of refraction creates a momentum threshold of $0.017 \mathrm{GeV} / \mathrm{C}$ for electrons, $4.8 \mathrm{GeV} / \mathrm{C}$ for pions, and $32 \mathrm{GeV} / \mathrm{c}$ for protons[15]. Relativistic particles entering the GC will produce a cone of Cherenkov radiation. This cone of light will be focused by a set of mirrors on the back plane 


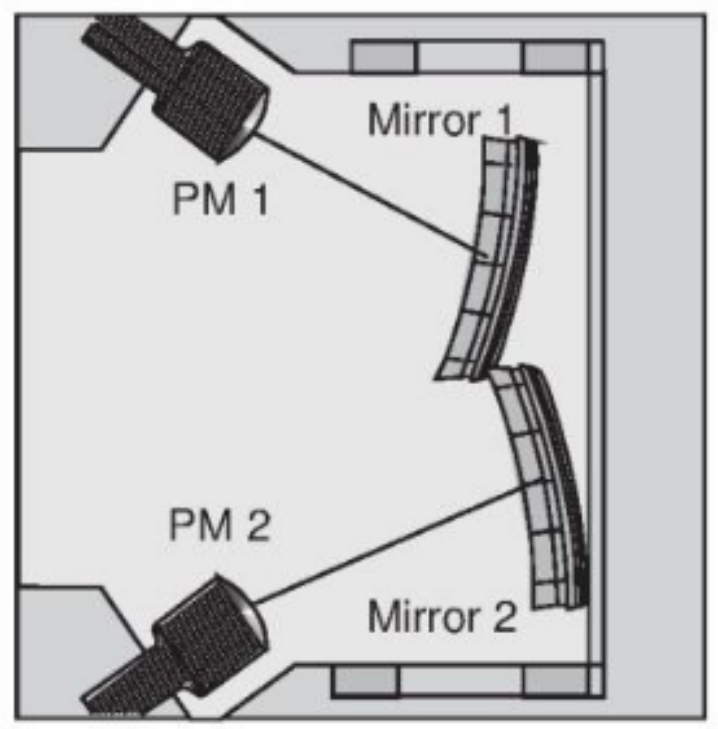

Figure 3.18: Top down depiction inside the GC [15].

of the GC. These mirrors direct the focused light onto a set of PMTs. A depiction of the GC from a top down perspective is shown in figure 3.18. The raw data recorded from the $\mathrm{GC}$ is in the form of raw ADC, or the size of the pulse seen by the PMT. In order to use this information, the ADC input needs to be calibrated. For the GC, two parts of the signal needs calibration. Each ADC channel sees a different amount of noise and signal background from electronic fluctuations. This signal background is defined as the ADC pedestal, and is the first calibration offset determined. Figure 3.19 shows the raw signal from one Cherenkov PMT. This signal shows the pedestal at approximately 5800 ADC channels. The pedestal is subtracted from the raw ADC signal to normalize the background electronic noise for all PMT-ADC pairs in the Cherenkov. The second calibration for the GC ADC signals is the photoelectron peak. The voltage used to power the PMTs in the Cherenkov is tuned before the experiment to allow the PMT to give the best pedestal to signal ratio while also persevering the life of the PMT and signal quality. This forces a different signal strength to be seen by each PMT for the same amount of light experienced in the chamber. The photo electric peak in the ADC signal is then normalized to the same value across all PMTs by a gain factor. 


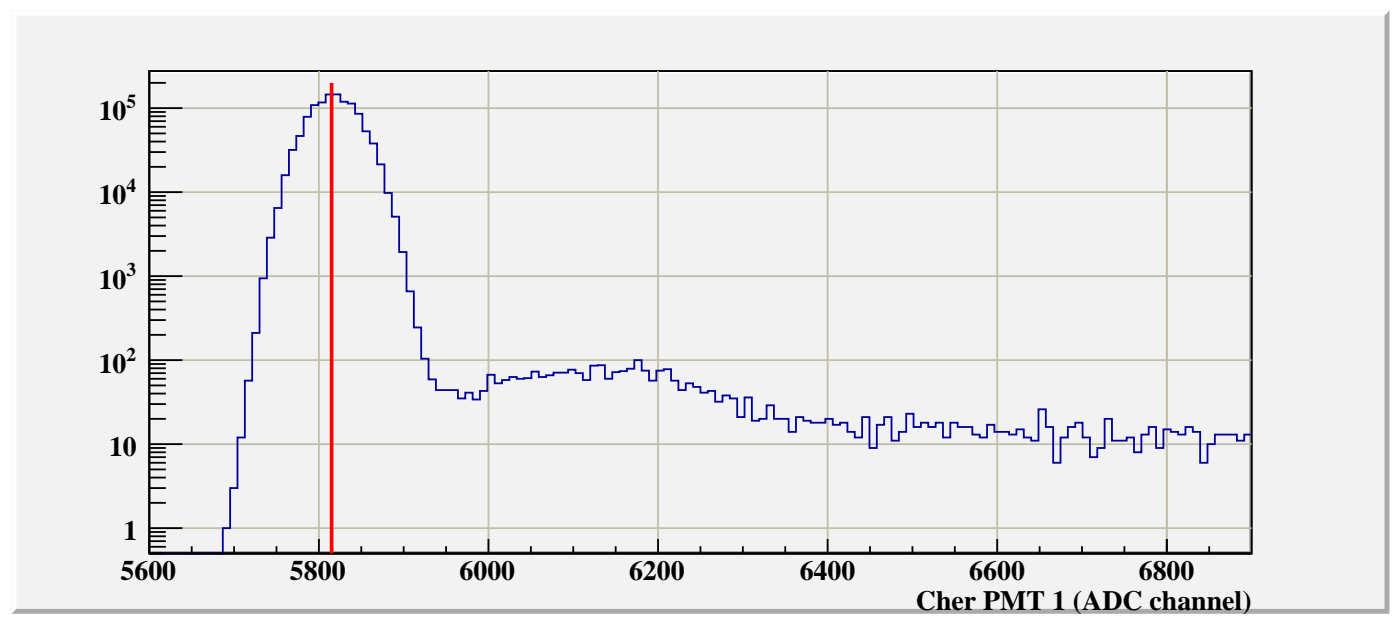

Figure 3.19: The raw signal captured from a single Cherenkov PMT, PMT[1], with a fit to the pedestal peak and a line draw to demonstrate its ADC channel number.

The GC's main task during an experiment is to help in the identification of particles (PID). During the MARATHON experiment, the GC was used to differentiate between negatively charged pions and electrons that passed through the detector. MARATHON used the GC in PID for data capture and analysis. During data capture, the GC signal was used in the formation of the main trigger. Forming the trigger with a requirement of a threshold in signal strength from the GC, allowed for the exclusion of many unwanted events. During the analysis of MARATHON data, pion suppression was done using the GC signal and signals from the calorimeter.

\subsubsection{Calorimeter}

The last detector in the spectrometer that particles interact with is the lead glass calorimeter. The Left HRS (LHRS) calorimeter system is made up of the preshower (PS) and shower (SH). The PS contains two columns of 24 blocks of lead glass with a PMT attached to the end. The SH has five columns, and each column as 16 blocks with a PMT. The right HRS' (RHRS) calorimeter system is constructed of the pion rejector 1 (PR1) and the pion rejector 2 (PR2). The two PRs on the RHRS consist of 34 blocks arranged in two, 17 block columns. 


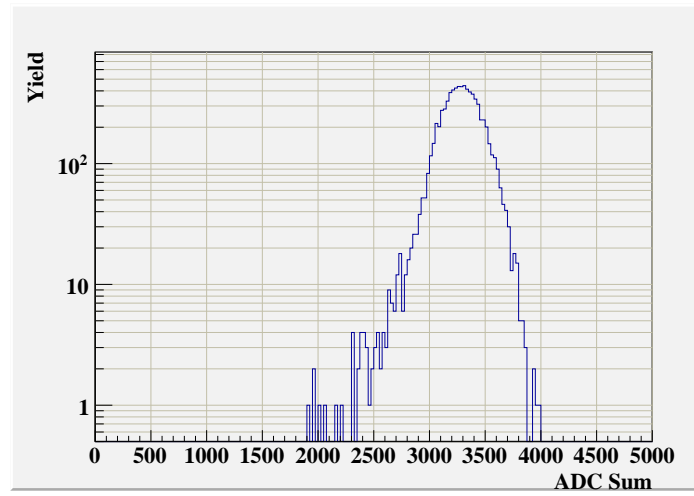

(a) ADC Sum

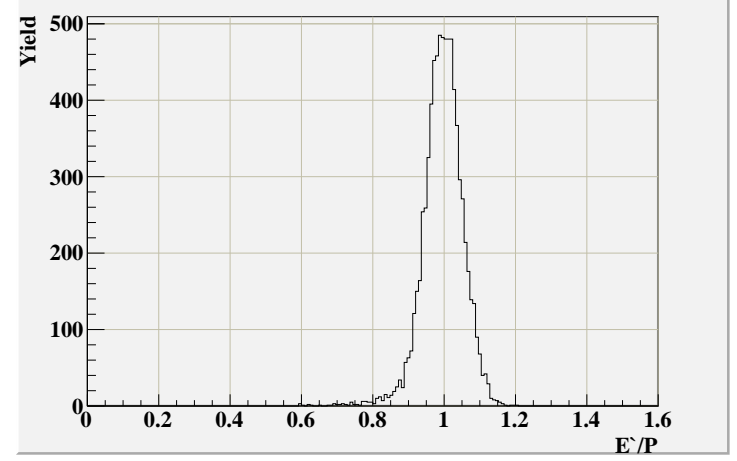

(b) $E^{\prime} / P$

Figure 3.20: Left: The sum of all ADC channels from the LHRS calorimeters. Right: The total energy deposited into the LHRS calorimeter scaled by the momentum setting. Electron cuts have been applied.

The calorimeters are used during the analysis process to help in PID. As high energy electrons pass through the dense leaded glass, the electron will lose its energy through bremsstrahlung radiation resulting in the emission of a photon. These photons begin an electromagnetic shower through the creation of positron-electron pairs. The shower of photons are detected by the PMTs attached to each block. The amount of energy contained in the scattered electron is directly proportional to the amount of photons generated during the shower.

The signal from the calorimeters is recorded as an ADC value. These ADC signals need to be calibrated in a similar way to the Cherenkov detector, subtracting the pedestal and determining the normalizing gain factor to match all PMT-ADC combinations. The total signal from the colorimeter can be seen in figure 3.20a. In order to use this ADC signal to help ID particles, the calorimeter needs an energy calibration. The calibration process uses a $\chi^{2}$ minimization. Equation 3.6 demonstrates the minimization technique applied. In this equation, $C_{j}$ is the calibration coefficient being determined for the calorimeter block $\mathrm{j}$. $\mathrm{Cal}_{i j}^{A D C}$ is the ADC signal received from block $\mathrm{j}$ during event $\mathrm{i}$, and $p_{i}$ is the momentum of 


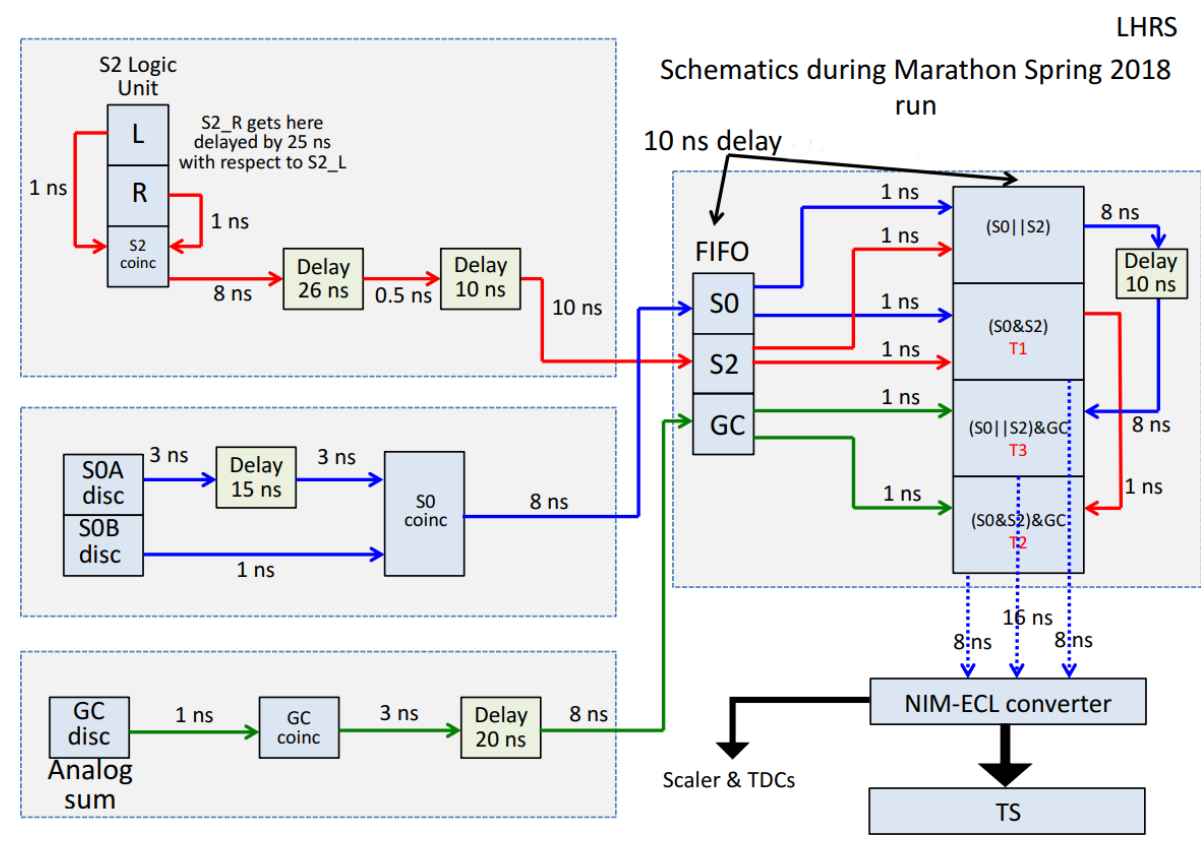

Figure 3.21: Scematic drawing of the trigger logic and timing for the MARATHON experiment [51].

the electron being detected.

$$
\frac{\partial \chi^{2}}{\partial C_{i}}=\sum_{i}^{\text {Events }}\left(\sum_{j}^{\text {Blocks }} C_{j} * C a l_{i j}^{A D C}-p_{i}\right)^{2}=0
$$

Using these calibration constants, the ADC signal in figure 3.20a can be turned into the calibrated data in the histogram show in figure 3.20b. This can be used to form PID selection cuts, removing any unwanted background events. The calorimeters design of being a total energy absorber is used to define the location of the electron selection cuts.

\subsection{Trigger Setup}

The MARATHON experiment designed three triggers to accept the most probable good electron events, while limiting the number of background events and preventing loss of efficiency due to electronic dead time. The design of these trigger are depicted in figure 3.21. Each trigger is built by the coincidence of signal between S0, S2 and the gas Cherenkov. Trigger 1 (T1) is the logical \& between the S0 and S2. This is used as a loose trigger to help 
test the detector timing and efficiencies. Trigger 2 (T2) is the main trigger used for good electron selection for the MARATHON experiment and is by combining T1 and GC with a logical \&. The addition of the GC helps remove many background pions and cosmic rays compared to T1. Trigger 3 (T3), a logical $\|$ between S0 and S2 and \& with the GC, was designed to help with the study of the efficiency of T1 and T2. The RHRS uses the same triggers, T4 copy of T1, T5 copy of T2, and T6 copy of T3.

The trigger signal from S0 is the logical and between the signals of the two PMTS of S0. S0A has an additional time delay. This delay forces the leading edge of S0B to be the leading edge of the output of the S0 coincidence. The trigger signal for S2 is built by a coincidence in both the left and right PMT attached to each bar of the scintillator. The signal from the right PMT is used as the leading edge of the coincidence signal. S2 has many bars so the trigger source is formed by a coincidence in any of the $\mathrm{S} 2$ scintillator bars. The S2 trigger signal has an additional delay compared to the S0 trigger signal. This delay forces S2R to be the leading edge of all the logical \& triggers The GC signal is formed by a sum of all the PMTs in the GC. If this sum meets some discriminator threshold, a trigger signal from the GC will be formed. The signals formed from the logic units for each of these trigger signals receive additional delays to prefect their timing in respect to each other. This tweaking of the timing spreads the trigger signals apart to help prevent the trigger signals from overlapping and allowing the recording of all possible triggers.

\subsection{Kinematic Settings}

The MARATHON experiment's goal is to measure cross section ratios of ${ }^{3} \mathrm{H},{ }^{3} \mathrm{He},{ }^{2} \mathrm{D}$, and $\mathrm{H}$ as a function of $x$. The MARATHON collaboration originally proposed to use the kinematics in table 3.1, allowing for the LHRS and RHRS to have mirror settings to expedite the rate of data collection at each position of $X$. The plan was to complete one kinematic setting and push the spectrometers out in angle from near 18 degrees at kinematic 1 to near 35 degrees at kinematic 16 while keeping the momentum settings of the spectrometers at 3.10 $\mathrm{GeV}$ for the 1st 15 settings, then decreasing the momentum to 2.9 for the last kinematic. 


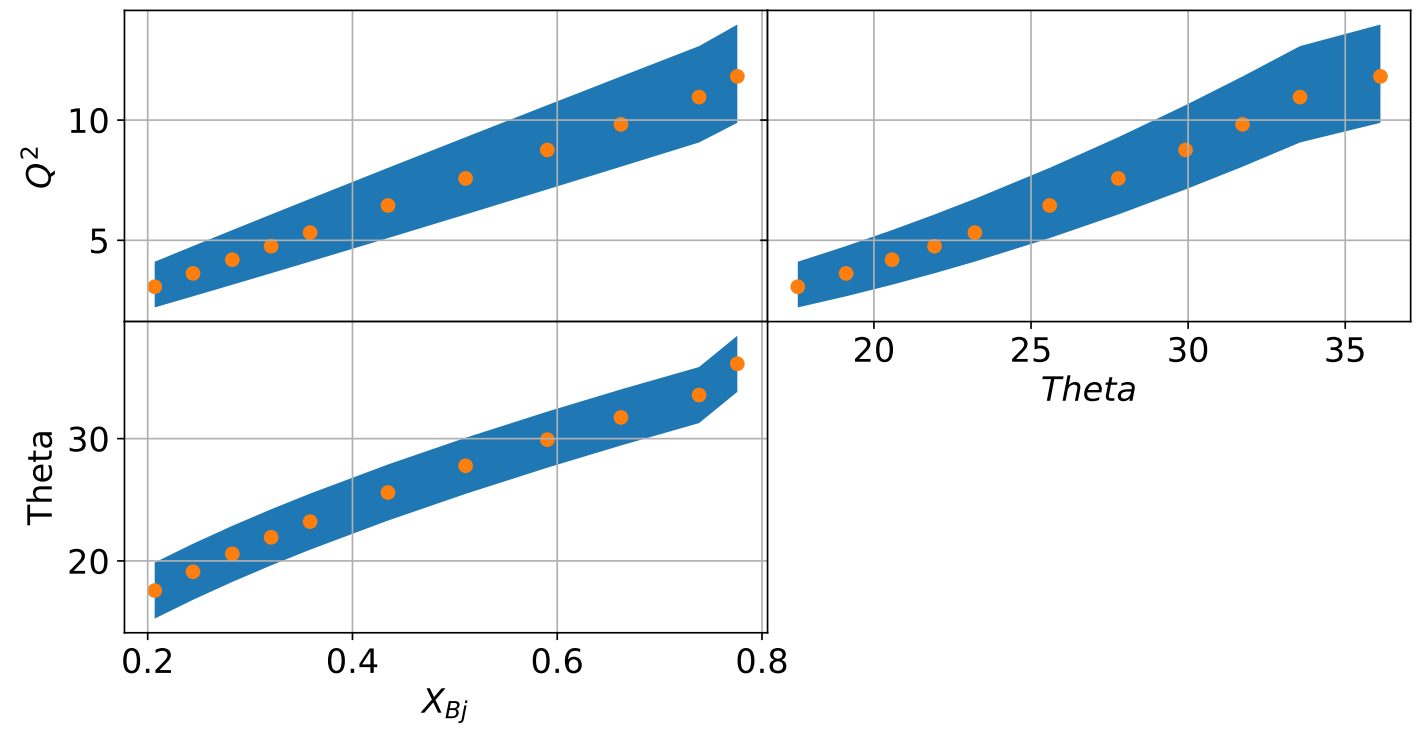

Figure 3.22: A kinematic coverage plot, demonstrating the $Q^{2}$ coverage for $x$ and Theta. Also the relationship between $x$ and Theta. The band around the points represents the approximate spectrometer acceptance in the y axis.

Due to time and physical constraints caused by issues with the running of all four halls simultaneously, the kinematics were adjusted to provided the best chance of reaching the statical goals at a large range in $x$. The angle setting for each kinematic were adjusted slightly. Most kinematics experience a slight decrease in angle setting to increase the rate of electron counting. During the first few days of running the MARATHON experiment the RHRS dipole experienced a power supply failure. This issue could not be resolved quickly. In order to complete our goal, the MARATHON experiment adjusted the kinematic plan to remove RHRS from running. The statical precision goal of the MARATHON experiment forced the collaboration to remove a few kinematic points from the plan. The kinematics that the MARATHON experiment was able to complete are listed in table 3.2. Figure 3.22 shows the kinematic coverage of the spectrometer for $x$, theta, and $\mathrm{Q}^{2}$ for the kinematics covered during the MARATHON experiment. After the new plan was solidified and data taking for the first few kinematics were complete, the RHRS was restored to services. The RHRS was then set to kinematic 16 for rest of the experiment. 
Table 3.1: Kinematics originally planned for the MARATHON experiment inlcuding an estimation of time required for three of the gas targets in hours. Estimations provided by John Arrington and Zhihong Ye[56].

\begin{tabular}{|l|l|l|l|l|l|l|l|l|l|}
\hline Kin. & $X_{B j}$ & $\begin{array}{l}\mathrm{W} 2 \\
\mathrm{GeV}^{2}\end{array}$ & $\begin{array}{l}\mathrm{Q} 2 \\
\mathrm{GeV}^{2}\end{array}$ & $\begin{array}{l}\mathrm{E}^{\prime} \\
\mathrm{GeV}\end{array}$ & $\begin{array}{l}\text { Theta } \\
\text { Degree }\end{array}$ & $\begin{array}{l}\mathrm{H} 2 \\
(\mathrm{~h})\end{array}$ & $\begin{array}{l}\mathrm{H} 3 \\
(\mathrm{~h})\end{array}$ & $\begin{array}{l}\text { He3 } \\
(\mathrm{h})\end{array}$ & $\begin{array}{l}\text { Total } \\
(\mathrm{h})\end{array}$ \\
\hline 1 & 0.23 & 12.30 & 3.41 & 3.10 & 18.19 & 0.28 & 0.45 & 0.28 & 1.02 \\
\hline 2 & 0.27 & 11.70 & 4.00 & 3.10 & 19.73 & 0.42 & 0.69 & 0.43 & 1.54 \\
\hline 3 & 0.31 & 11.11 & 4.60 & 3.10 & 21.15 & 0.62 & 1.03 & 0.62 & 2.27 \\
\hline 4 & 0.35 & 10.52 & 5.19 & 3.10 & 22.49 & 0.90 & 1.50 & 0.89 & 3.29 \\
\hline 5 & 0.39 & 9.92 & 5.78 & 3.10 & 23.76 & 1.29 & 2.17 & 1.27 & 4.73 \\
\hline 6 & 0.43 & 9.33 & 6.37 & 3.10 & 24.97 & 1.85 & 3.13 & 1.81 & 6.79 \\
\hline 7 & 0.47 & 8.74 & 6.97 & 3.10 & 26.12 & 2.66 & 4.52 & 2.57 & 9.75 \\
\hline 8 & 0.51 & 8.14 & 7.56 & 3.10 & 27.23 & 3.80 & 6.53 & 3.66 & 13.99 \\
\hline 9 & 0.55 & 7.55 & 8.15 & 3.10 & 28.30 & 5.52 & 9.56 & 5.27 & 20.36 \\
\hline 10 & 0.59 & 6.96 & 8.75 & 3.10 & 29.34 & 5.12 & 14.19 & 7.70 & 30.01 \\
\hline 11 & 0.63 & 6.37 & 9.34 & 3.10 & 30.34 & 12.12 & 21.39 & 11.41 & 44.92 \\
\hline 12 & 0.67 & 5.77 & 9.93 & 3.10 & 31.31 & 18.56 & 33.08 & 17.35 & 68.99 \\
\hline 13 & 0.71 & 5.18 & 10.53 & 3.10 & 32.26 & 29.08 & 52.35 & 26.98 & 108.41 \\
\hline 14 & 0.75 & 4.59 & 11.12 & 3.10 & 33.18 & 47.19 & 85.80 & 43.47 & 176.46 \\
\hline 15 & 0.79 & 3.99 & 11.71 & 3.10 & 34.08 & 87.73 & 150.03 & 74.76 & 306.51 \\
\hline 16 & 0.83 & 3.40 & 12.30 & 3.10 & 34.96 & 155.36 & 287.74 & 141.21 & 584.30 \\
\hline
\end{tabular}

Table 3.2: Kinematic settings used during the MARATHON experiment. Kinematic 1-15 for LHRS, and kinematic 16 using RHRS. The good electron count is in units of thousands.

\begin{tabular}{|l|l|l|l|l|l|l|l|l|}
\hline kin & $\mathrm{X}$ & $\mathrm{W} 2$ & $\mathrm{Q} 2$ & $\mathrm{E}^{6}$ & theta & D2 count & He3 count & H3 count \\
\hline 1 & 0.22 & 11.89 & 3.07 & 3.1 & 17.58 & 94.0 & 93.0 & 124.3 \\
\hline 2 & 0.26 & 11.33 & 3.62 & 3.1 & 19.11 & 109.0 & 103.0 & 120.5 \\
\hline 3 & 0.3 & 10.76 & 4.19 & 3.1 & 20.58 & 121.0 & 78.0 & 101.1 \\
\hline 4 & 0.34 & 10.2 & 4.76 & 3.1 & 21.93 & 78.0 & 64.0 & 69.8 \\
\hline 5 & 0.38 & 9.63 & 5.32 & 3.1 & 23.21 & 25.0 & 39.0 & 39.3 \\
\hline 7 & 0.46 & 8.51 & 6.45 & 3.1 & 25.59 & 40.0 & 40.0 & 41.2 \\
\hline 9 & 0.54 & 7.38 & 7.57 & 3.1 & 27.78 & 36.0 & 36.0 & 35.5 \\
\hline 11 & 0.62 & 6.2 & 8.76 & 3.1 & 29.92 & 29.0 & 27.0 & 27.6 \\
\hline 13 & 0.7 & 5.13 & 9.82 & 3.1 & 31.73 & 23.0 & 23.0 & 23.0 \\
\hline 15 & 0.78 & 4.0 & 10.96 & 3.1 & 33.56 & 21.0 & 23.0 & 22.8 \\
\hline $16^{*}$ & 0.82 & 3.51 & 11.82 & 2.90 & 36.12 & 24.2 & 23.9 & 24.6 \\
\hline
\end{tabular}




\section{Chapter 4}

\section{Data Analysis}

The goals for the MARATHON experiment are to determine the EMC effect for the two $\mathrm{A}=3$ systems ${ }^{3} \mathrm{He}$ and ${ }^{3} \mathrm{H}$, extract the $F_{2}^{n} / F_{2}^{p}$, and calculate the $d / u$ quark distribution ratios. The goal of this analysis is to determine the EMC effect for the two $\mathrm{A}=3$ systems via the ratio of measured cross sections. The cross-section of a scattering interaction is the probability of that event happening. In order to measure the probability of an event happening, a ratio has to be calculated between the number of those events versus the number of times that event could have happened. This analysis will use data from the HRSs, beamline detectors, and target information to measure the cross-section of ${ }^{3} \mathrm{He}$ and ${ }^{3} \mathrm{H}$ for kinematics of $0.2<x<$ 0.82. The cross section can be extracted experimentally in bins of $x$ using the following equation:

$$
\frac{d \sigma}{d E^{\prime} d \Omega}=\frac{(N-B G)}{\mathscr{L} \cdot \epsilon \cdot \Delta E^{\prime} \Delta \Omega \cdot A\left(E^{\prime}, \theta\right)} .
$$

The number of counted electrons $(\mathrm{N})$ have to be corrected for any background events (BG) and for inefficiencies $(\epsilon)$ in the detectors, data collection, particle identification, and analysis. The electron yield $(Y=(N-B G) / \epsilon)$ is normalized by the luminosity $(\mathscr{L})$ and detector acceptance in momentum and scattering angle $\left(A\left(E^{\prime}, \theta\right)\right)$. The $x$ bin for this cross section is a function of the momentum and solid angle phase space $\left(\Delta E^{\prime} \Delta \Omega\right)$. This chapter will discuss the process of analyzing raw data received from the detector to extract the raw cross-section in bins of $x$. This process starts with the Hall A analysis software. 
Hall A at JLab uses an analysis software (Analyzer) that is built on top of CERN ROOT. The Analyzer is used to decode raw data signals received from TDCs, ADCs, and scalars into meaningful results. The decoding process uses raw data from the detectors in the HRSs and on the beam line to create an event. This event is assigned a track if applicable, and the event's signal from the detectors are stored into a ROOT file. This ROOT file contains the raw and calibrated detector data from each signal, the tracking information, and physics variables calculated via the calibrated data and tracking information. The analysis of an event begins with reconstructing its track.

\subsection{Tracking}

The Analyzer uses calibrated VDC data to calculate the track of an event. The detector calibration was discussed in chapter 3. An event's track contains information on trajectory and location of a particle as it travels through the spectrometer. The track determined from the VDCs is in reference to the detector coordinate system. The Analyzer uses an optics matrix to relate the tracking information between all coordinate systems used in the Hall A analysis process. The coordinate systems will be briefly discussed in this section. A more complete guide with in-depth discussion of the coordinate systems is presented in [31]. The coordinate system definitions and relations are obtained from [31, 4, 62].

- Hall Coordinate System(HCS): The intersection of the electron beam and the vertical symmetry axis of the target system defines the origin of the HCS. This allows for $\hat{z}$ to point along the beam line towards the beam dump and $\hat{y}$ is up.

- Target Coordinate System (TCS): The TCS is unique to the individual HRS. The $\hat{z}$ axis of the TCS is defined by a line perpendicular to the surface of the spectrometers sieve slit aligned with the midpoint of the center hole. $z_{t g}$ points away from the target towards the spectrometer. Nilange Liyanage states in reference [62], "In the ideal case where the spectrometer is pointing directly at the hall center and the sieve slit is perfectly centered on the spectrometer, the $\mathrm{z}_{t g}$ axis passes through the hall center." Using the ideal case, the origin of the TCS is defined by a set distance from the sieve 


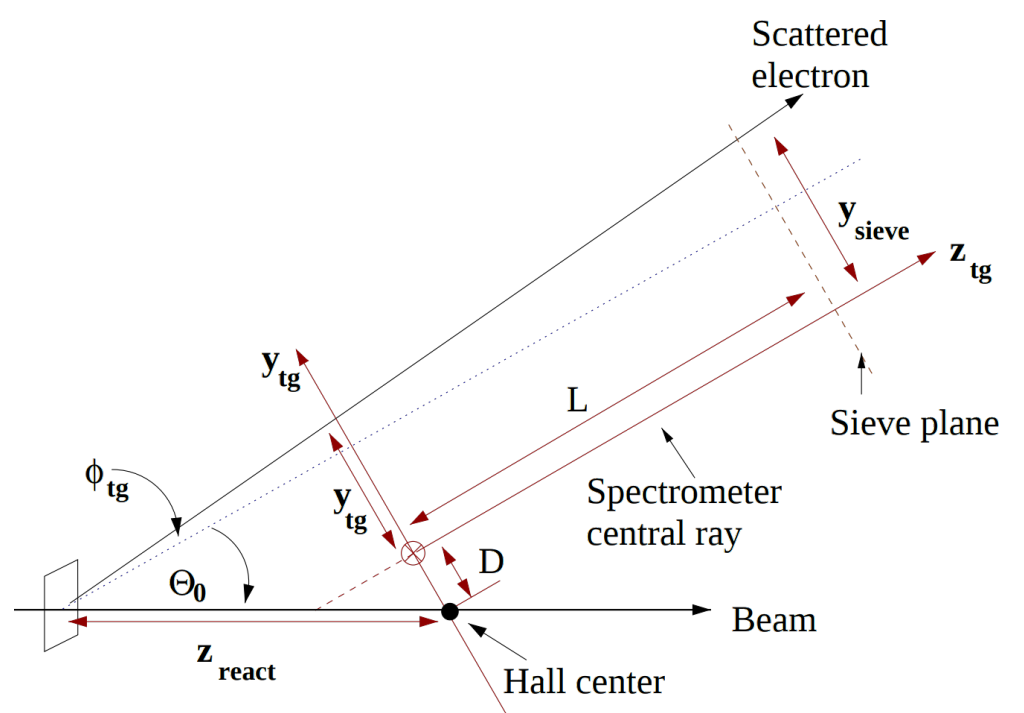

Figure 4.1: The TCS for an electron scattering event as seen from above. The event happens at $z_{\text {react }}$ distance from the Hall center. $\mathbf{L}$ is the distance from the Hall center to the sieve plane. $\mathbf{D}$ is the horizontal displacement of the spectrometer axis from the ideal position. $\Theta$ is the spectrometer's central angle [62].

surface. For the Left HRS, the TCS origin is $1.181 \mathrm{~m}$ from the sieve, and for the Right HRS it is $1.178 \mathrm{~m}$. In the ideal case, the TCS origin is the HCS origin. Figure 4.1 shows the TCS with a an electron scattering event from a foil.

- Detector Coordinate System (DCS): The origin of the DCS is at the intersection of wire 184 of the U1 and V1 planes of the first VDC. $\hat{y}$ is parallel to the short symmetry axis of the lower VDC [31]. $\hat{z}$ is vertically up, perpendicular to the vdc planes. $\hat{x}$ points away from the center of curvature of the dipole. The DCS values are calculated by using the intersection points of the 4 VDC planes and the spacial information of the VDC planes.

- Transport Coordinate System (TRCS) at the focal plane: Rotating the DCS clockwise around its y-axis by $45^{\circ}$ generates the TRCS. The TRCS can be expressed by DCS variables.

- Focal Plane Coordinate System (FCS): The FCS is used to transport an event's track from the DCS to the TCS. The FCS is determined by rotating the DCS around its y-axis by the angle between the local central ray and the $\hat{z}$ axis of the DCS. The 
local central ray is defined as a ray with $\theta=\phi=0$ for the corresponding relative momentum $\frac{\Delta_{p}}{p}(\delta)[62]$. In the calculation of the FCS, the offsets of the VDCs are used to correct any misalignments.

The Analyzer provides two spatial coordinates and two angular coordinates for each event. These coordinates are determined by data received via the VDCs and decoded into the DCS. $x_{d e t}$ and $\theta_{\text {det }}$ are the particle's position and tangent of the angle made by its trajectory along the dispersive direction. $y_{\text {det }}$ and $\phi_{\text {det }}$ are the particle position and tangent of the angle perpendicular to the dispersive direction [62]. Using rotation matrices and other transfer definitions these DCS coordinates are converted to the FCS. The analyzer uses a calibration matrix to transport the FCS to the TCS. The first order approximation of the matrix can be defined as:

$$
\left[\begin{array}{l}
\delta \\
\theta \\
y \\
\phi
\end{array}\right]_{t g}=\left[\begin{array}{cccc}
\langle\delta \mid x\rangle & \langle\delta \mid \theta\rangle & 0 & 0 \\
\langle\theta \mid x\rangle & \langle\theta \mid \theta\rangle & 0 & 0 \\
0 & 0 & \langle y \mid y\rangle & \langle y \mid \phi\rangle \\
0 & 0 & \phi y|y\rangle & \langle\phi \mid \phi\rangle
\end{array}\right]\left[\begin{array}{c}
x \\
\theta \\
y \\
\phi
\end{array}\right]_{f p}
$$

The relationship between the focal plane variables and the target coordinates our conventionally expressed in a set of tensors, defined as $\mathrm{Y}_{j k l}, \mathrm{~T}_{j k l}, \mathrm{P}_{j k l}$, and $\mathrm{D}_{j k l}$. These tensors are polynomials in $\mathrm{x}_{f p}$ and are optimized to the 5 th order. They can be used to relate the FCS to the TCS by the following relations:

$$
\begin{aligned}
y_{t g} & =\sum_{j, k, l} Y_{j k l} \theta_{f p}^{j} y_{f p}^{k} \phi_{f p}^{l} \\
\theta_{t g} & =\sum_{j, k, l} T_{j k l} \theta_{f p}^{j} y_{f p}^{k} \phi_{f p}^{l} \\
\phi_{t g} & =\sum_{j, k, l} P_{j k l} \theta_{f p}^{j} y_{f p}^{k} \phi_{f p}^{l} \\
\delta_{t g} & =\sum_{j, k, l} D_{j k l} \theta_{f p}^{j} y_{f p}^{k} \phi_{f p}^{l}
\end{aligned}
$$

The elements of the optics matrix used for the transporting from the FCS to the TCS are part of the tensors, $\mathrm{Y}_{j k l}, \mathrm{~T}_{j k l}, \mathrm{P}_{j k l}$, and $\mathrm{D}_{j k l}$. The elements of the optics matrix are calculated 
via an optics calibration using a sieve slit and multi foil optics target. The procedure for calculating those matrix elements are presented in reference [62].

Along with the tracking information in the TCS, the analyzer also provides the location of the reaction vertex, the scattering angle for the electron event, and the momentum of the scattered electron from the tracking information. The reaction vertex along the beam line, $\mathrm{z}_{\text {react }}$, is the location where the scattering event happened in the HCS. $\mathrm{z}_{\text {react }}$ can be found via the following relationship:

$$
z_{\text {react }}=\frac{-\left(y_{t g}+\text { Spect_Offset }_{y}\right)+x_{\text {beam }}\left(\cos \left(\Theta_{0}\right)-\phi_{t g} \sin \left(\Theta_{0}\right)\right)}{\cos \left(\Theta_{0}\right) \phi_{t g}+\sin \left(\Theta_{0}\right)}
$$

The relationship for $\mathrm{z}_{\text {react }}$ includes terms for the offset due to the mis-pointing of the spectrometer (Spect_Offset $y$ ), the offset of the beam at the point of intersection with the target $\left(\mathrm{x}_{\text {beam }}\right)$, and the setting for the spectrometer central angle $\left(\Theta_{0}\right)$. The scattering angle of the electron, $\theta_{\text {scat }}$, is determined by a relationship with the TCS angle coordinates $\theta_{t g}$ and $\phi_{t g}$ and the angle setting of the spectrometer. The relationship used to calculate the scattering angle from the target coordinates is described in reference [4], as:

$$
\theta_{\text {scat }}=\arccos \left(\frac{\cos \left(\theta_{0}\right)-\phi_{t g} \sin \left(\theta_{0}\right)}{\sqrt{1+\theta_{t g}^{2}+\phi_{t g}^{2}}}\right)
$$

The accuracy of the TCS angles $\theta_{t g}$ and $\phi_{t g}$ determine the accuracy of the measured scattered angle. The precise measurement of the scattering angle is crucial to every electron counting experiment. In order to provide accurate measurement of the scattering angle, a set of measurement surveys are completed. These surveys measure the:

- the target position,

- the spectrometer central angle,

- the mispointing of the spectrometer nominal central ray from the hall center,

- the position of the sieve-slit center with respect to the nominal central ray, 
- the position of the BPMs with respect to the ideal beam line [4].

The results from the surveys have an approximate systematic uncertainty of $0.5 \mathrm{~mm}$ due to equipment uncertainties. The contribution of all the measurement uncertainties added in quadrature provide an approximate $0.6 \mathrm{mrad}$ uncertainty to the overall measurement of the scattered angle [4].

The momentum of the scattered electron is also calculated via tracking information. $\delta_{t g}$, the relative momentum of an event, is used in the calculation of the momentum of a particle traveling through the spectrometer. $p$, the absolute momentum for an event, is determined through, $p=p_{0}\left(1+\delta_{t g}\right)$. $p_{0}$ is the central momentum setting of the spectrometer. This momentum setting of the spectrometer is determined via a measurement of the magnetic field , $\mathrm{B}_{\text {dipole }}$, of the HRS dipole. The determination of the central momentum from the magnetic field is given by a third order polynomial,

$$
p_{0}=\sum_{i=1}^{3} \Gamma_{i} B_{0}^{i}
$$

$\Gamma$ is a set of spectrometer constants calculated to an accuracy of $4 \times 10^{-4}$. The left HRS constants are $\Gamma_{1,2,3}(2702,0,-1.6)$, and the right HRS constants are $\Gamma_{1,2,3}(2698,0,-1.6)$ in units of $\mathrm{MeV} / \mathrm{T}^{i}[4]$. Once the analyzer produces a track for an event, the event can be included, or excluded, for future consideration. In the next section, I will discuss the selection of events for continued analysis.

\subsection{Electron Selection}

The extraction of the electron scattering cross section requires the counting of scattered electrons from a target. In order to count the number of scattered electrons, data are analyzed on an event by event basis. The event is passed through a number of criteria (cuts) to determine the identity and source of the scattered event. The cuts used for this analysis are separated into two different types, acceptance and particle identification (PID). The acceptance cuts are used to limit the source location and trajectory of the particle through 


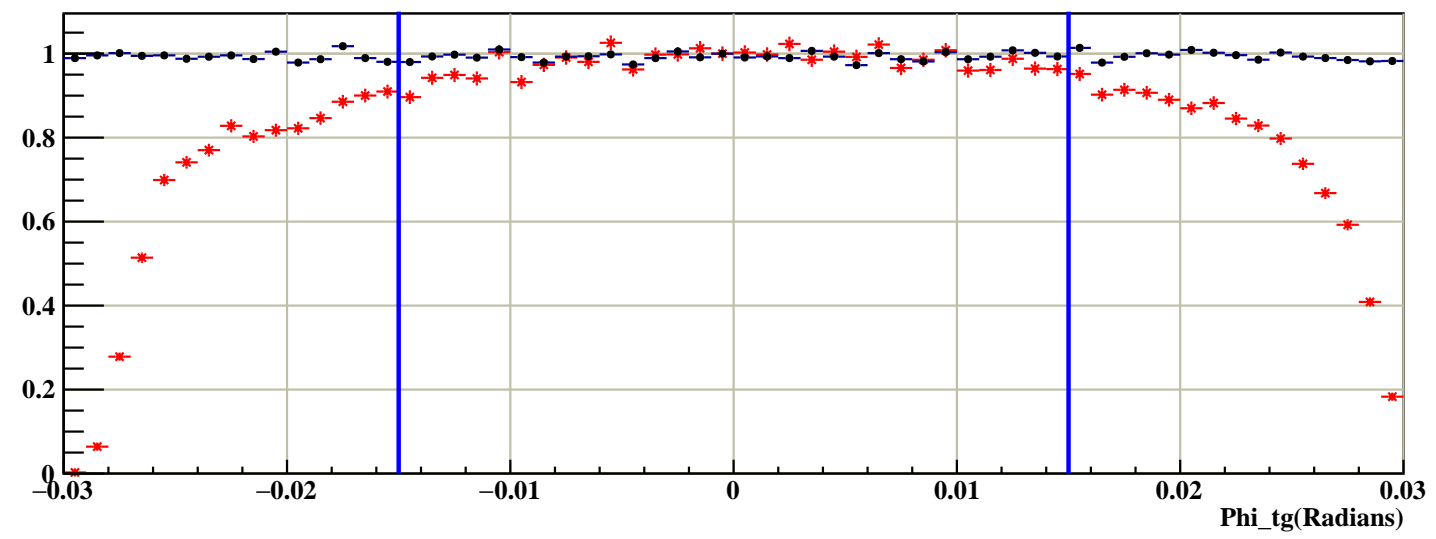

Figure 4.2: Simulation of events to demonstrate the acceptance of the spectrometer. Black events have been generated with no acceptance effects, Red points include a cut to only allow events that traverse the entire spectrometer. The Blue lines are example locations of cuts used in event selection to exclude the edge of the acceptance.

the detector to provide greater accuracy for the reconstruction of the particle's scattered angle, momentum, and scattering vertex location. Cuts are also used to identify the particle and the type of scattering interaction it experienced.

\subsubsection{Acceptance Cuts}

The HRS was designed to accept particles within a range of $\delta, y_{t g}, \theta_{t g}, \phi_{t g}$, and the vertex location along the beam line. The acceptance of the spectrometer is well known near the central ray. Due to the geometry of the spectrometer's magnets, the distribution of accepted electrons at the edge of the acceptance is not well understood. This is shown in figure 4.2. Applying cuts in the target variables creates an area of acceptance that is well understood. The acceptance cuts used for this analysis are:

- $-0.035>=\delta_{t g}<=0.035$

- $-0.04 \mathrm{rad}>=\theta_{t g}<=0.04 \mathrm{rad}$

- $-0.025 \mathrm{rad}>=\phi_{t g}<=0.025 \mathrm{rad}$

A cut is applied to the acceptance variable for the reaction location, Vertex z. The target cells are constructed of aluminum with thin end caps. Many electrons that are sent to the target scatter off the aluminum end caps. Comparing the empty cell to the cells filled with 
gas provides an observable comparison between the yield of events from the end caps of the cells to the yield of events from the gas in the cell. Section 4.4.1 continues the discussion on mitigation of contamination due to events scattered off the end caps.

- $-0.07 \mathrm{~m} \quad>=$ Vertex $\mathrm{Z}<=0.09 \mathrm{~m}$

The analysis software assigns an event a track if the signal in the VDCs meet the required criteria. It is possible for the signal received by the VDC to produce multiple tracks for one event. Events that produce multiple tracks are cut out via a cut. These events are removed due the uncertainty of the correct track and position within the acceptance of the spectrometer. Section 4.3.4 contains a discussion of the efficiency of the cut applied to remove multi track events.

- Number of tracks $==1$

\subsubsection{Identification Cuts}

The process of capturing data from the two HRSs begins with the firing of a trigger. The trigger design for MARATHON focused on triggering for electrons and reducing the amount of other particles. Figure 3.21 describes the design of MARATHON's main trigger and efficiency triggers. MARATHON's main trigger, trigger 2, consist of a $(S 0 \& S 2) \& G C$. This was discussed in section 3.5. Using the scintillators as a trigger allows the capture of data from charged particles that pass through the detector. These particles are mainly electrons and pions. The addition of the GC to the trigger allows for a first pass cut on unwanted particles. Applying a MARATHON trigger cut to the data narrows the sample of non-electrons that need to be analyzed. The trigger data is stored bitwise by the analysis software to allow for the capture of every trigger that fired for the event. The trigger cut applied for this analysis requires the event to fire the MARATHON trigger but does not exclude events that produce multiple triggers. This is accomplished by bitwise comparing the trigger to 1 bit shifted to the left by 2 .

- MARATHON trigger (bit) \& $(1<<2)$ 


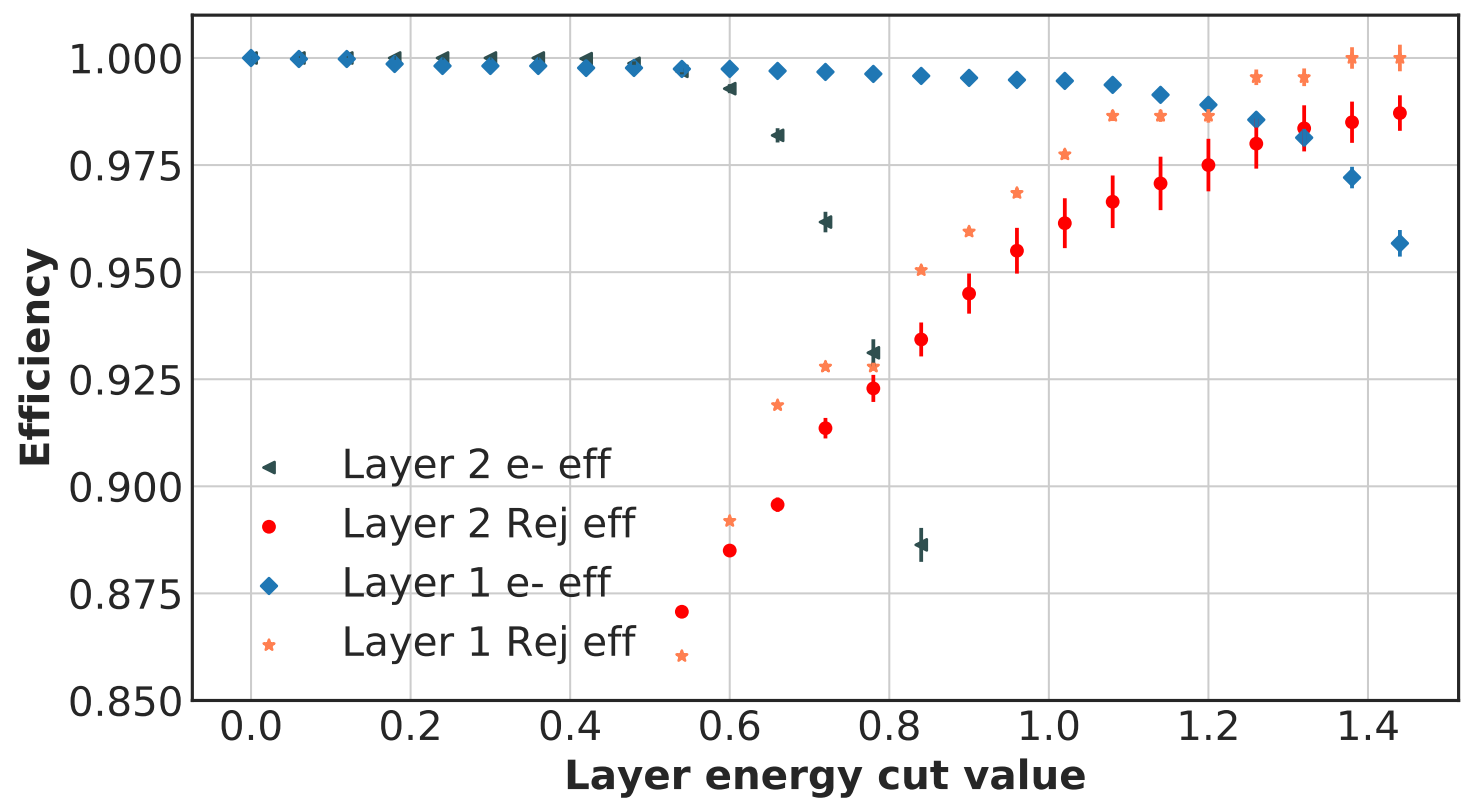

Figure 4.3: A scan of the PID efficiency for a cut in each layer of the calorimeters.

The largest source of contamination for the MARATHON experiment is negatively charged pions. These pions are removed through software cuts made in the total signal from the ten Cherenkov PMTs (photomultiplier tubes) and the energy deposited into the blocks of both layers of the calorimeter. Electrons can be identified by their behavior in the spectrometer. High-quality electrons will track through the entire detector stack to deposit most of their energy into the total calorimeter system and creating a large amount of light in the Cherenkov. An efficiency scan is used to determine the best location for the cuts in the calorimeters and Cherenkov. An efficiency scan for the two layers of the calorimeters is shown in figure 4.3. The scan covers a large range of possible energy deposition in a layer of the calorimeter calculating both the efficiency of rejecting pions and of counting electrons. The description of how the efficiency is calculated can be found in section 4.3.2.

- Total Cherenkov ADC sum > 1800

- Calo. Layer 1 Energy $>1 \mathrm{GeV}$

- Calo. Layer 2 Energy $\quad>0.6 \mathrm{GeV}$

The electrons that have passed all of the previously discussed cuts are tools used to study the scattering interaction and the components of the scattering interaction that produced 
the resulting event. The kinematics used for the MARATHON will produce events in the DIS, resonance, and quasi-elastic regimes of the scattering spectrum. A cut is applied to the invariant mass of the scattered event to only include events that are deeply inelastic.

$$
\text { - } \mathrm{W}^{2}>2.5 \mathrm{GeV}^{2}
$$

Events that pass all the criteria discussed in this section are considered to be a good electron and will be counted for the extraction of the cross section.

\subsection{Efficiencies}

The high resolution spectrometers are capable of detecting a myriad of particles that track through the detectors. The design of an experimental trigger uses the properties of the individual detectors to capture relevant events. Many accidentals, background, and unwanted events trigger the data acquisition system, and some good electrons are missed by our DAQ. The removal of these unwanted events takes place during analysis via software cuts. Restricting the applicable signal from certain detectors through different cuts allows for the rejection of background particles and prevents contamination in the yield extraction. The efficiency of the HRSs and the software cuts applied will be discussed in this section.

\subsubsection{Computer and electronic Livetime}

The signals from events that fire the DAQ travel through electronics including amplifiers and logic modules on their way to be recorded by the TDCs and ADCs. The processing of these signals requires time at each stage. During that time another event will be discarded due to limitations in the hardware. This time when the DAQ system cannot handle another event is known as the dead-time of the system. Livetime therefore is the percentage of time when an event can be recorded. The lost events need to be accounted for during the analysis process. The livetime of the DAQ system for the MARATHON experiment was measured by determining the percentage of events that were recorded relative to the number of events that fired the corresponding trigger. The livetime for the MARATHON experiment depended on the rate of events. The livetime during the highest rate kinematic was determined to be 
Table 4.1: Livetime for each kinematic setting during the MARATHON experiment calculated using trigger 2 .

\begin{tabular}{|l|l|l|l|l|l|l|l|l|}
\hline Kin & 1 & 2 & 3 & 4 & 5 & 7 & 9 & $11,13,15$ \\
\hline LiveTime & 0.947 & 0.969 & 0.981 & 0.986 & 0.992 & 0.996 & 0.997 & 0.998 \\
\hline
\end{tabular}

0.947, and climbs to 0.998 for the highest angle setting. Listed in table 4.1 are the calculated values for livetime at each kinematic.

\subsubsection{Particle Identification Efficiency}

The contamination of pions is a large concern for the counting of scattered electrons. Software cuts are used to remove the pions from the electron count. The efficiency of the PID cuts will be discussed in this section. Plotting the signal in the Cherenkov versus the energy deposited into both layers of the calorimeter allows for visual representation of the sampling cuts made in the efficiency studies, which can be seen in figure 4.4.

$$
\begin{aligned}
G E_{\text {sample }} & =\text { Known electron sample from tight cut } \\
G E_{\text {pass }} & =G E_{\text {sample }} \text { and pass identification cut } \\
\text { Electron }_{\text {eff }} & =\frac{G E_{\text {pass }}}{G E_{\text {sample }}}
\end{aligned}
$$

The efficiencies of the spectrometer's particle identification (PID) detectors were determined by using the first calorimeter layer, the second calorimeter layer, and the Cherenkov to provide samples of good electrons and other particles. The PID efficiency of the individual detectors was determined using equation 4.10. The good electron sample for calculating the efficiency of the single detector was defined by sampling through the other two detectors. Sampling through the two layers of the calorimeter is shown in the top left plot of figure 4.5 for the first layer of the calorimeter and top right for the second layer. The Cherenkov good electron sample is shown in the bottom plot of figure 4.5. The electron sample from the Cherenkov is contaminated by delta rays and a combination of unknown particles. These unidentified background particles are known to be relativistic due to the amount of light seen 


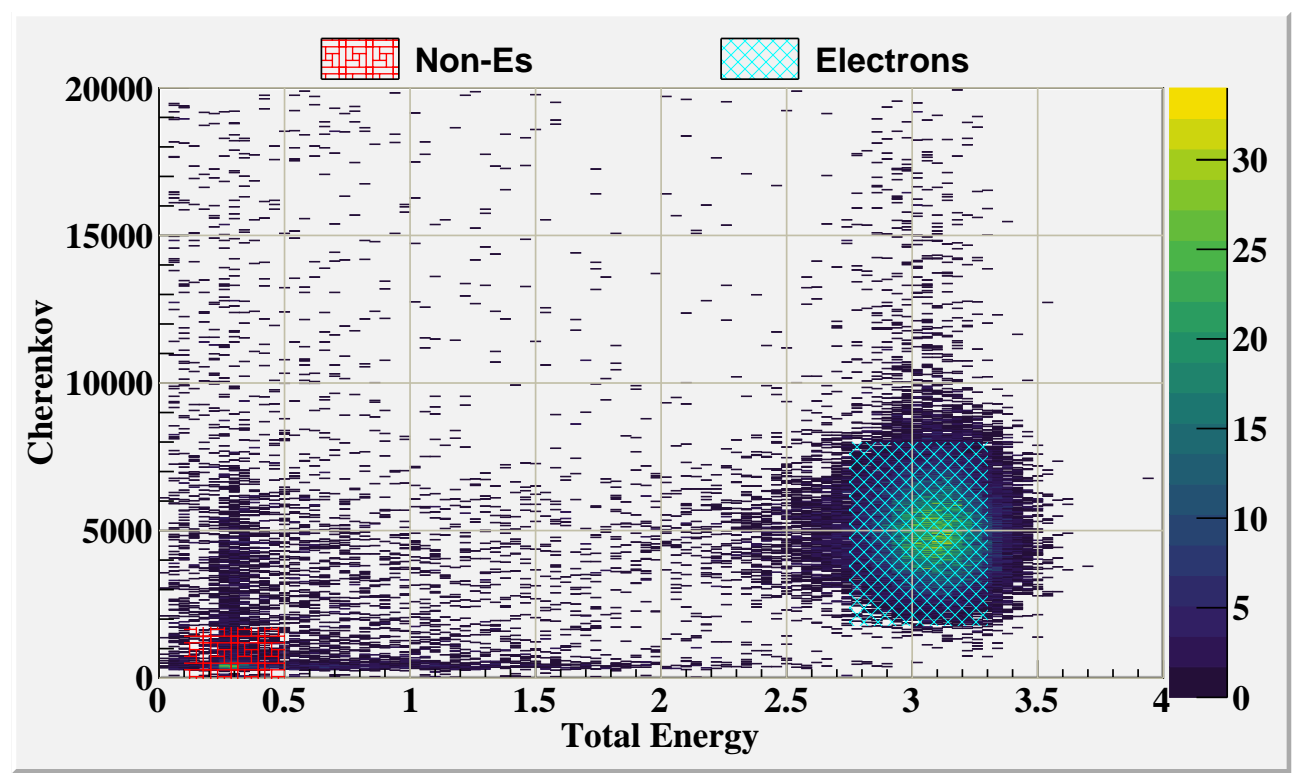

Figure 4.4: Two dimensional plot of the Cherenkov sum versus Total Energy deposited, including electron sampling in teal and non-electron sampling in red.

in the Cherenkov. However, the events do not deposit enough energy into the calorimeter system to be considered as a good electron that scattered from our target through the detector. Using sampling in one layer of the calorimeter and the Cherenkov, these unwanted low energy particles are rejected from sampling for efficiency calculations. The electron selection PID efficiency for the three PID detectors was determined at each kinematic setting to be approximately $98 \%$. The efficiency was determined to be independent of the kinematic setting. Only small fluctuations were seen during the study. These small changes are due to a decrease in statistics, and all of the results fall within statistical uncertainty of being independent of kinematic setting. Figure 4.6 displays the efficiency for the Cherenkov and both layers of the calorimeter at different kinematics for different targets. The non-electron suppression efficiency was determined as part of this PID efficiency study to ascertain how many back ground particles leak into our sample of good electrons after cuts are made. The suppression efficiency of the Cherenkov suffered due to the contamination of the relativistic low energy particles. Combining the two calorimeter detectors with the Cherenkov increased the overall suppression efficiency for the spectrometer to $99.9 \%$ over the entire kinematic range of the MARATHON experiment. 


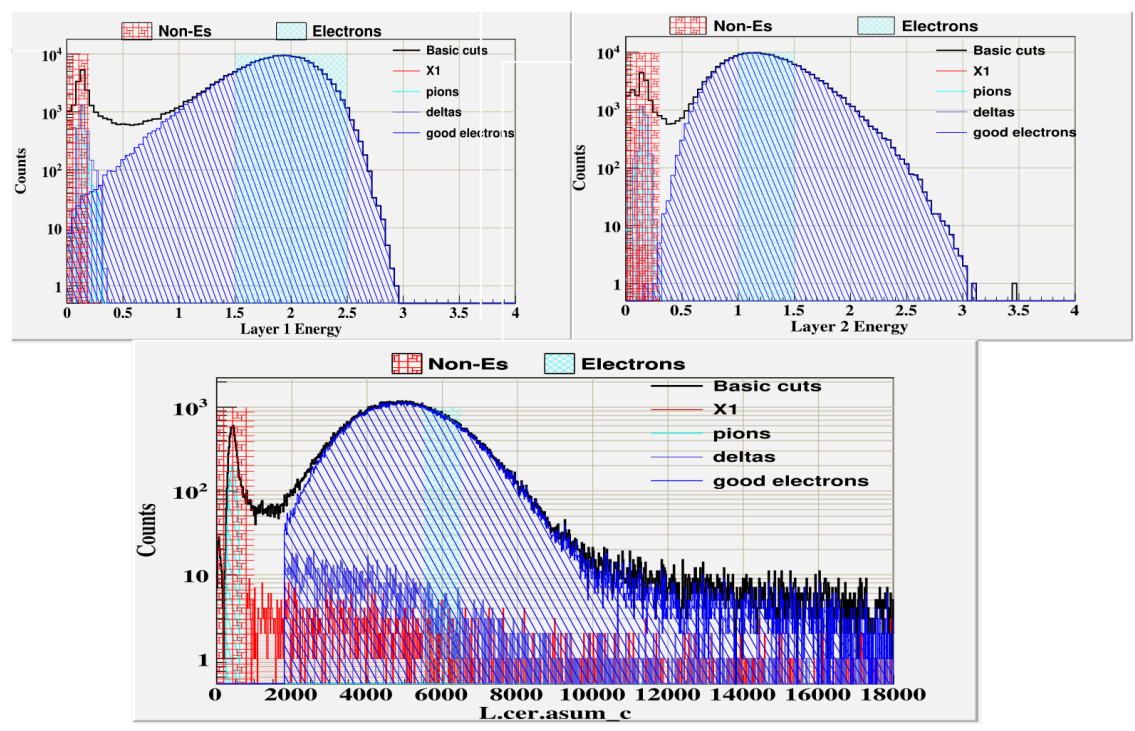

Figure 4.5: Electrons and other background particles identified via cuts in the total calorimeter and the gas Cherenkov shown in the individual layers of the calorimeters (top) and the Cherenkov (bottom). Sampling cuts for Electrons in teal and Non-Electrons in red.

\subsubsection{Trigger Efficiency}

Due to inefficiencies of the electronics, logic, and detectors an event can produce a false trigger or a high quality electron may not fire the main trigger. A low threshold in the Cherenkov signal allows for an inclusive trigger limiting the overall number of quality electrons missed, but results in a large quantity of non-electron triggers. Software PID cuts prevent the contamination of non-electrons in the yield calculation. The tight PID software cuts remove the false positive inefficiency from the trigger design and is then considered in the PID efficiencies. The trigger inefficiency caused by missed high quality electrons was then calculated by sampling the high quality electrons in trigger $1,(S 0 \& S 2)$. This ties the efficiency of trigger 2 with the performance of the scintillators. The efficiency of the two scintillating planes in conjunction is calculated by using sampling in trigger $3,(S 0 \mid S 2) \& G C$ with strict PID cuts in both layers of the calorimeters and requiring a hit in the Cherenkov. The two scintillator planes in conjunction have an efficiency greater than $99.7 \%$ for all kinematics. Combining the trigger efficiency of the main trigger shown in figure 4.7 with the performance of the scintillators gives an overall efficiency for the trigger of the MARATHON experiment of greater than $99.6 \%$. 


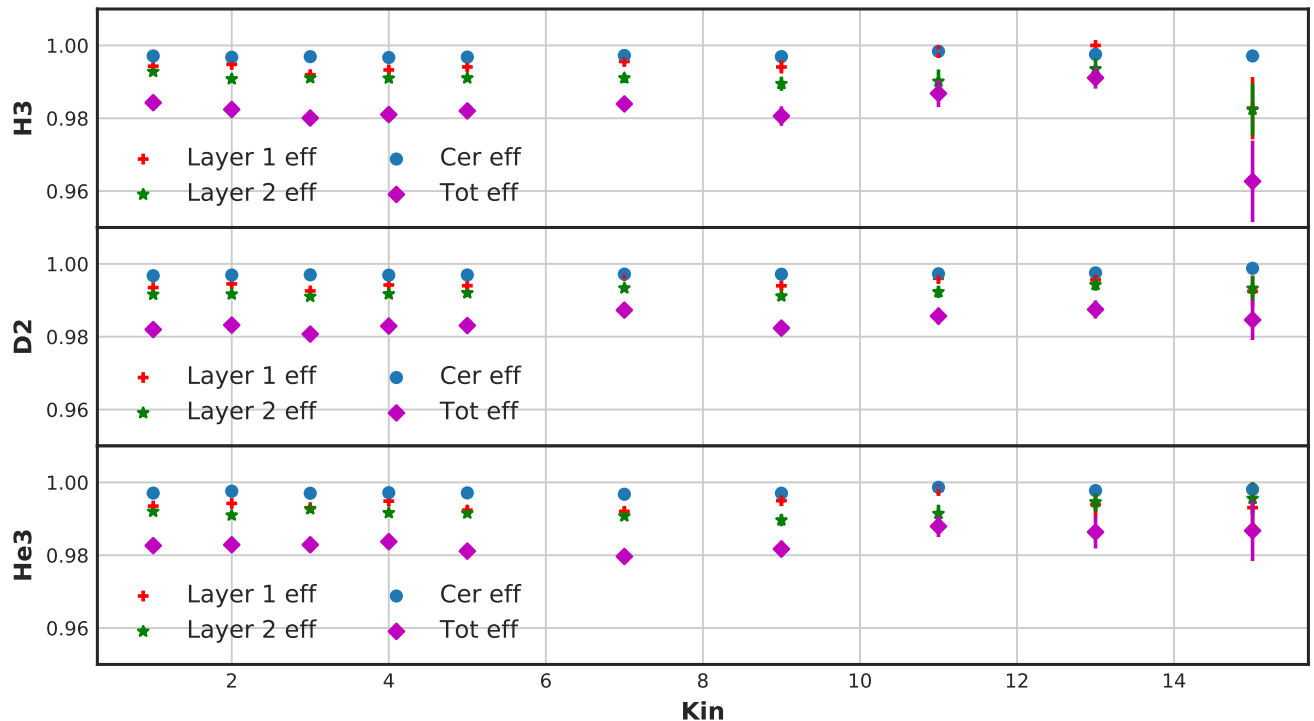

Figure 4.6: The PID efficiency for the Cherenkov and both layers of the calorimeter,including the overall total PID efficiency for each of the gas targets at all of the kinematics.

\subsubsection{Tracking Efficiency}

Particles that travel through our detector could have originated from sources wanted or unwanted. In order to control the source of the scattered electrons, we use a particle's track to identify its source. The signals received via the VDC are used to produce a track from the target to end of the spectrometer. The largest source of inefficiency for the VDCs is incorrectly identified tracks. High quality electrons that traverse the spectrometer should only have one good track, calculated via the tracking package in the analysis software. The capability of the VDCs to determine a good electron event's one good track is known as the one track efficiency for the VDCs. Quantitatively, the one track efficiency $\left(\epsilon_{V D C}\right)$ can be obtained via:

$$
\epsilon_{V D C} \equiv \frac{N_{1 t r a c k}}{N_{\text {all }}}
$$

Where the number of good electron events that have one good track is defined as $N_{1 \text { track }}$, and $N_{\text {all }}$ are all of the electrons rather they have a good track or not. The good electron selection is made via PID cuts in the calorimeter and Cherenkov, and cuts in the ADC and TDC of the scintillators. Direct cuts in the signal of the scintillators were made to include 


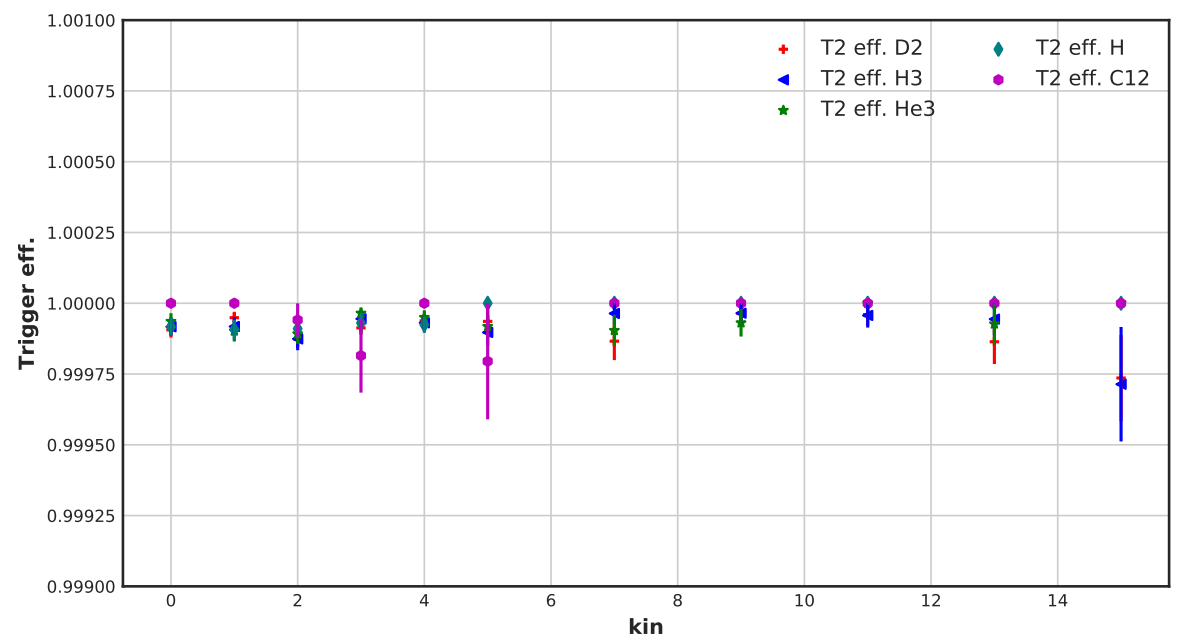

Figure 4.7: Trigger efficiency of trigger 2 for different targets at all kinematics calculated via sampling from trigger 1.

the nominal acceptance cuts, which are produced through tracking software. A graph of the tracking efficiency of the HRS for different kinematic settings during the MARATHON experiment is shown in figure 4.8 for the three gas targets. The efficiency of the VDCs is independent of the angle of the spectrometer. The uniform tracking efficiency across all kinematics is expected and helps eliminate any concerns of the performance of the VDCs during the experiment.

\subsection{Background Subtraction}

The purpose of this analysis is to study the DIS cross sections of ${ }^{2} \mathrm{D},{ }^{3} \mathrm{He}$, and ${ }^{3} \mathrm{H}$. The sample of scattered events used to determine the cross section of a given nuclear target then needs to be cleaned of any contamination produced from other targets and processes. The electrons detected by the spectrometers can be electrons that scattered from our chosen target, scattered from a source other than our target, or produced through process other than DIS scattering. The two sources of contamination for the MARATHON experiment are events scattered from the aluminum end caps of the target cell and pair produced electrons via photon interactions. The ${ }^{3} \mathrm{H}$ gas will experience beta decay, that produces ${ }^{3} \mathrm{He}$. This ${ }^{3} \mathrm{He}$ that contaminates the ${ }^{3} \mathrm{H}$ cell will also be addressed in this section. 


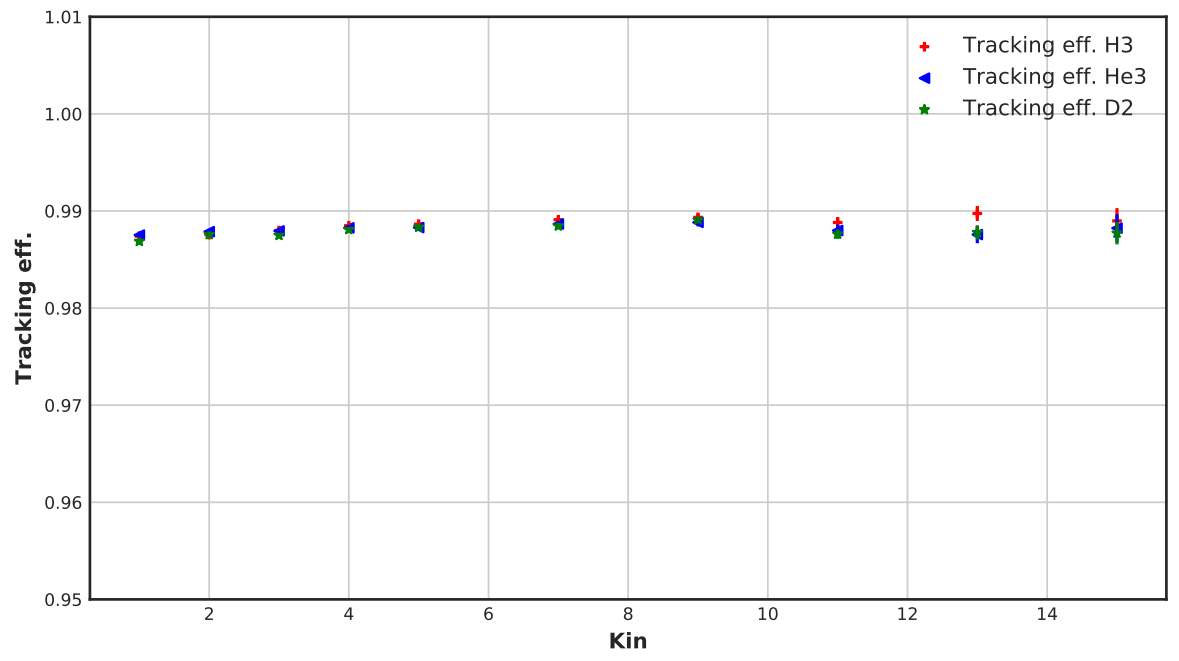

Figure 4.8: Tracking efficiency of the VDCs for different targets at all kinematics.

\subsubsection{End Caps}

The target cells used during the MARATHON experiment are shown in figure 3.13. The majority of the events from the end caps can be removed easily via a cut in the reconstructed quantity of reaction vertex along the beam axis. The relatively high density thickness of the aluminum end caps causes a large amount of end cap contamination. The majority of the electrons that scatter from the end caps can be removed through software cuts in the reaction vertex along the beam axis $(\mathrm{z})$. Shown in figure 4.9 is a comparison of the reaction vertex of the electron events between the gaseous targets and the empty cell target at kinematic 4 . The yield is normalized by the number of events in the histogram to remove any bias from the amount of time of beam on target. The empty target results in figure 4.9, demonstrate the normalized yield for electrons scattering off of the aluminum windows of our target cell. Using the reconstructed vertex location of the scattering origin, the vast majority of the events from the windows can be removed. This vertex cut is shown by the two vertical blue lines. Only events that lie within these two lines are considered good electrons from our chosen target.

The empty cell vertex z distribution does have content within the vertex cut. These events that remain after the cut are corrected for via an end cap contamination factor. This factor 


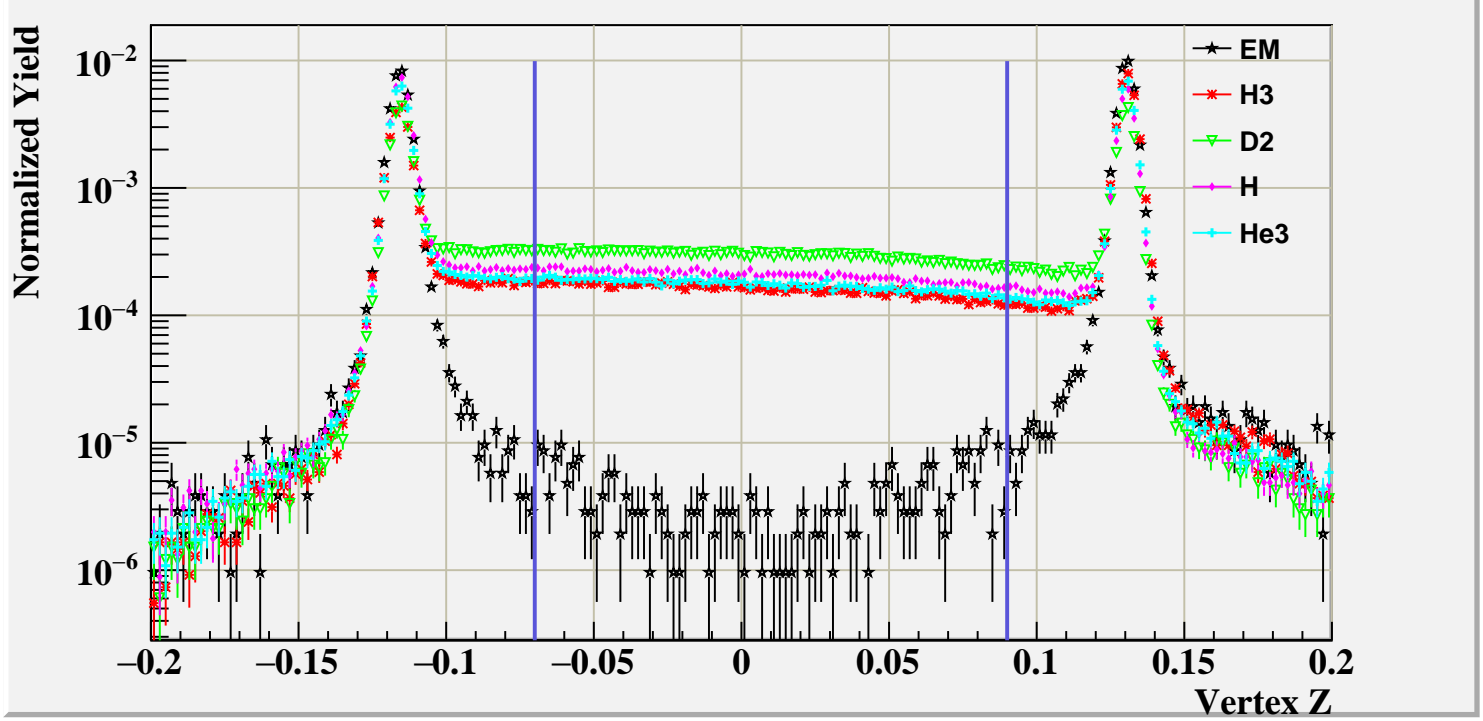

Figure 4.9: Comparison of the scattering vertex along the $\mathrm{z}$ axis for the empty target (EM) and the gas targets at kin. 4.

is calculated by determining the ratio of the number of good electrons that scatter from the empty cell and from each gas cells, resulting in ratio of $\left(\frac{\text { Yield }_{E C}}{\left(\text { Yield }_{\text {Gas }}+\text { Yield }_{E C}\right)}\right)$. Where the subscript EC denotes events from the end caps. The sum of the scattered events from the gas and from the EC is the value counted from production data on the gas target cells. The correction factor applied to the yield calculation is defined as:

$$
E C C=1-\left(\frac{\text { Yield }_{E C}}{\left(\text { Yield }_{\text {Gas }}+\text { Yield }_{E C}\right)}\right) \equiv \frac{\text { Yield }_{\text {gas }}}{\left(\text { Yield }_{\text {Gas }}+\text { Yield }_{E C}\right)}
$$

\subsubsection{Pair Produced Electrons}

The high energy scattering interaction used to create deep inelastic scattering events can produce high energy photons and pions. The high energy photons that have energy greater than $1.022 \mathrm{MeV}$ can convert into $\mathrm{e}^{+} \mathrm{e}^{-}$pairs when the photons interact with a medium. A correction for the number of background electrons produced via a pair production process was calculated by determining the amount of positrons produced for all targets and kinematics. The yield of positrons was measured for kinematics one through five. The results were used to construct a function to determine the amount of contamination at high $x_{B j}$ kinematics. 


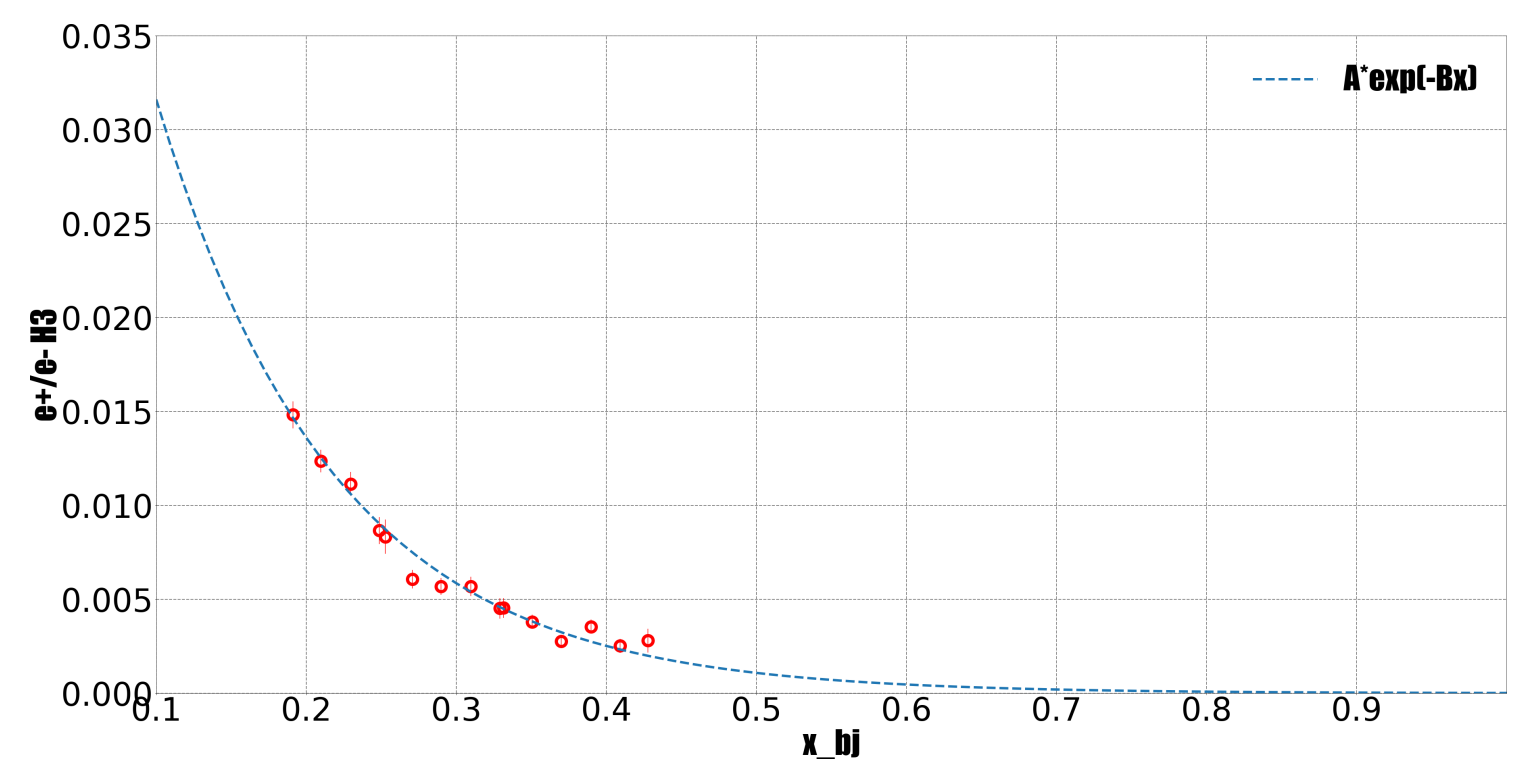

Figure 4.10: The ratio of positron events to electrons for ${ }^{3} \mathrm{H}$ [82].

Figure 4.10 shows the amount of positron contamination for ${ }^{3} \mathrm{H}$ and an exponential fit to extrapolate over the entire ranged in $x_{B j}$ for the MARATHON experiment.

\subsubsection{Beta Decay of ${ }^{3} \mathrm{H}$}

${ }^{3} \mathrm{H}$, a radioactive isotope of hydrogen will beta decay to ${ }^{3} \mathrm{He} .{ }^{3} \mathrm{H}$ has a half-life of 4500 \pm 8 days [63]. The gas cell used to contain ${ }^{3} \mathrm{H}$ for the experiment was filled on October 23, 2017. The initial ${ }^{3} \mathrm{H}$ thickness density of our ${ }^{3} \mathrm{H}$ cell was $0.077 \pm 0.001$ grams per $\mathrm{cm}^{2}$. The ${ }^{3} \mathrm{H}$ in our cell is diatomic and decays via two channels[85]. The possible decay channels and their branching probabilities are shown in equation 4.12. In DIS interactions, the molecular effects are ignored due to the size of the probe in a DIS scattering event which allows for the different channels to be treated as one.

$$
\begin{aligned}
{ }^{3} \mathrm{H}_{2} & \rightarrow\left({ }^{3} \mathrm{H}^{3} \mathrm{He}\right)^{+} \\
{ }^{3} \mathrm{H}_{2} & \rightarrow\left({ }^{3} \mathrm{H}\right)^{+}+\left({ }^{3} \mathrm{He}\right)^{+}
\end{aligned}
$$

The amount of ${ }^{3} \mathrm{H}$ and ${ }^{3} \mathrm{He}$ in our ${ }^{3} \mathrm{H}$ cell will change in respect to the time since the filling of the cell. Equations 4.13 and 4.14 describe the amount of ${ }^{3} \mathrm{H}$ and ${ }^{3} \mathrm{He}$ in the ${ }^{3} \mathrm{H}$ cell as a 


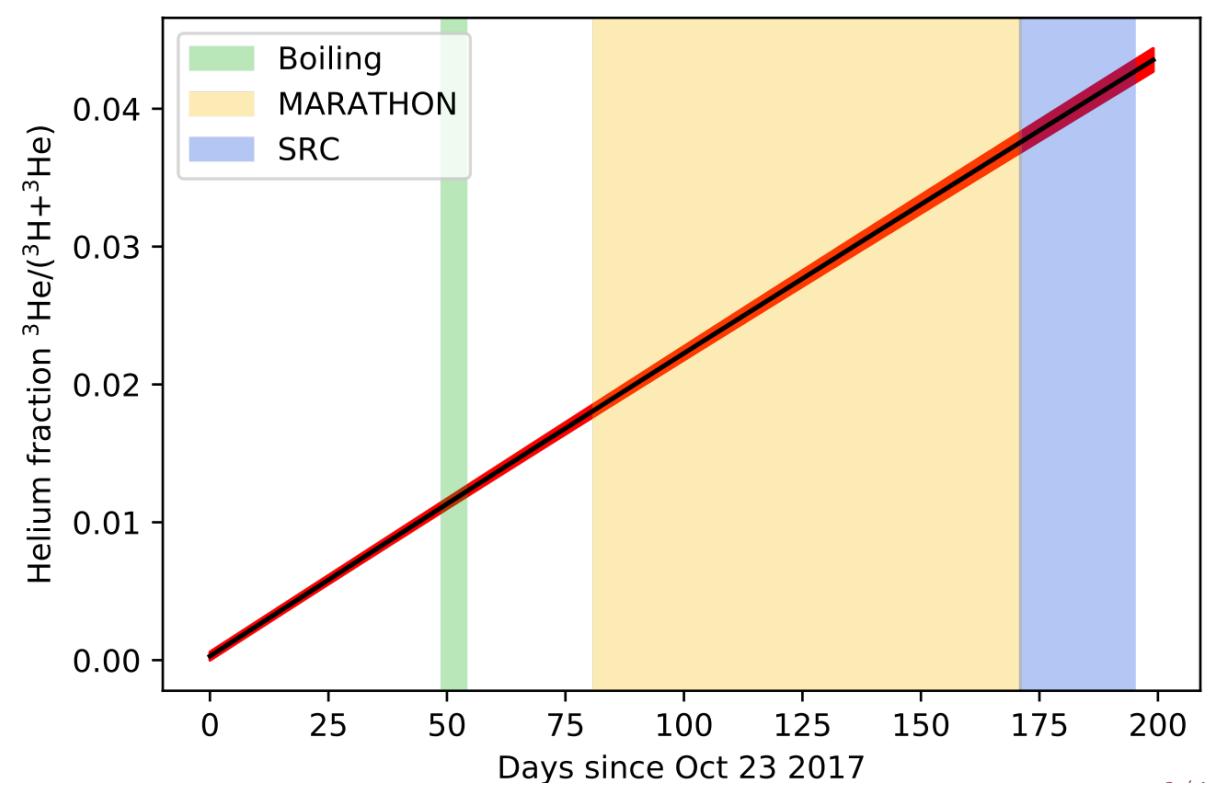

Figure 4.11: The amount of ${ }^{3} \mathrm{He}$ in the ${ }^{3} \mathrm{H}$ cell in reference to the total amount of material in the cell as a function of time. Included are bands of time for different sections of the ${ }^{3} \mathrm{H}$ run group's plan [60].

function of the time since fill date and the original amount of ${ }^{3} \mathrm{H}$ and ${ }^{3} \mathrm{He}$ in cell at filling. In equations 4.13 and $4.14, n_{T}\left(n_{H}\right)$ is the time dependent amount of ${ }^{3} \mathrm{H}\left({ }^{3} \mathrm{He}\right)$, and $n_{T}^{0}\left(n_{H}^{0}\right)$ is the amount of ${ }^{3} \mathrm{H}\left({ }^{3} \mathrm{He}\right)$ in the cell at time of filling. $\mathrm{t}$ is the time since the cell was filled and $\tau$ is the mean livetime of ${ }^{3} \mathrm{H}$.

$$
\begin{aligned}
& n_{T}=n_{T}^{0} e^{-t / \tau} \\
& n_{H}=n_{H}^{0}\left(1-e^{-t / \tau}\right),
\end{aligned}
$$

As time passes the amount of ${ }^{3} \mathrm{He}$ increases, the contamination becomes a non-negligible effect on the yield of scattered electrons. The fraction of ${ }^{3} \mathrm{He}$ in the ${ }^{3} \mathrm{H}$ can reach up to $3 \%$ for the data from the end of the MARATHON experiment. This ${ }^{3} \mathrm{He}$ fraction as a function of time is shown in figure 4.11, with the period for running the MARATHON experiment labeled as a color band.

$$
Y=\frac{\sum N_{i}}{\sum Q_{i} n_{i}},
$$




$$
\begin{gathered}
Y_{\text {raw }}=\frac{\sum\left(T_{i}+H_{i}\right)}{\sum Q_{i}\left(n_{T, i}+n_{H, i}\right)} \\
\left\langle f_{H}\right\rangle \equiv \frac{\sum Q_{i} f_{H, i}}{\sum Q_{i}}
\end{gathered}
$$

The events that scattered from ${ }^{3} \mathrm{He}$ in our ${ }^{3} \mathrm{H}$ cell need to be subtracted from the measured yield. The yield from any target is defined as the number of electrons per charge weighted scattering centers. The yield is shown in equation 4.15 and defined as the number of counted events $\left(N_{i}\right)$ per possible scattering changes, or charge $\left(Q_{i}\right)$ times number of scattering centers $\left(n_{i}\right)$. Data were recorded in many runs, so a sum over runs $(i)$ is required to get the total yield. The subtraction factor is calculated by breaking down the yield from the ${ }^{3} \mathrm{H}$ cell as the addition of the yield from ${ }^{3} \mathrm{H}\left(Y_{T}\right)=\Sigma\left(T_{i} / Q_{i} n_{i}\right)$ and ${ }^{3} \mathrm{He}\left(Y_{H}\right)=\Sigma\left(H_{i} / Q_{i} n_{i}\right)$ in the ${ }^{3} \mathrm{H}$ cell as shown in equation 4.16. The correction for the beta decay is defined in equation 4.18. It can be determined by expanding equation 4.16 out and solving for the ${ }^{3} \mathrm{H}$ yield from the ${ }^{3} \mathrm{H}$ cell. Where $\left\langle f_{H}\right\rangle$ is the charge weighted ${ }^{3} \mathrm{He}$ fraction defined in equation 4.17 [17]. The ${ }^{3} \mathrm{He}$

fraction $f_{H, i}$ is the ratio of ${ }^{3} \mathrm{He}$ scattering centers in the ${ }^{3} \mathrm{H}$ cell to total number of scattering centers.

$$
Y_{T}=Y_{\text {raw }}\left(\frac{1}{1-\left\langle f_{H}\right\rangle}\right)-Y_{H}\left(\frac{\left\langle f_{H}\right\rangle}{1-\left\langle f_{H}\right\rangle}\right)
$$

The beta decay correction depends on kinematic setting. The charge weighted average correction factor is calculated for each kinematic. The correction is then applied to the yield in each bin of that kinematic.

\subsection{Luminosity}

The luminosity $(\mathscr{L})$ is the amount of possible scattering interactions. $\mathscr{L}$ is defined as the product of the number of incoming beam particles, the target particle density, and the target's thickness [76]. The MARATHON experiment took many runs of data for each kinematic. The luminosity of each runs was calculated and summed together to determine the luminosity for each kinematic.

$$
\mathscr{L}_{\text {Run }}=\left(\frac{Q_{e} \cdot T_{\text {thick }} \cdot \rho_{c} \cdot N_{a}}{\text { AtomicMass }}\right) \quad \mathscr{L}_{\text {kin }}=\sum_{i}^{\text {Runs }} \mathscr{L}_{i}
$$


Table 4.2: Table of gas target density thickness and uncertainty [66].

\begin{tabular}{lcc}
\hline Target & Thickness $\left(\mathrm{mg} / \mathrm{cm}^{2}\right)$ & Uncertainty $\left(\mathrm{mg} / \mathrm{cm}^{2}\right)$ \\
\hline${ }^{3} \mathrm{H}$ & 85.1 & 0.8 \\
${ }^{3} \mathrm{He}$ & 53.4 & 0.6 \\
${ }^{2} \mathrm{D}$ & 142.2 & 0.8 \\
\hline
\end{tabular}

The calculation of the $\mathscr{L}$ requires data from the BCMs to determine the total charge sent to the target during a run. This is converted to number of electrons to produce $Q_{e}$. The targets density thickness $\left(\mathrm{T}_{\text {thick}}\right.$ ) was provided by the JLab's target group as part of their target report [66]. Table 4.2 list the target thickness and uncertainty in the target thickness measurements. Avogadro's number and the atomic mass of the target makes the luminosity have units of electrons per $\mathrm{cm}^{2}$. Then applying a conversion for $\mathrm{cm}$ to $\mathrm{nb}$ gives the luminosity units commonly used for cross section extractions.

Due to the fluid nature of the gas targets, a target's density will fluctuate with temperature. A temperature control system uses cryogenic gas and heaters to control the temperature of the target. Power supplied to the target from the incident beam heats the gas causing temperature fluctuations and local density variations in the target gas. A dedicated density change study was performed by S.N. Santiesteban and S. Alsalmi [78].

$$
\rho_{c}=p_{0}+p_{1} \cdot I+p_{2} \cdot I^{2}
$$

Their study showed a quadric dependence on the density of the gas targets with respect to the current applied. The equation used to determine the correction applied to the target's density for a given current is shown in equation 4.20 . Where $\mathrm{p}_{i}$ are the parameters for the quadric fit, and $I$ is the current. The correction parameters for each target are listed in table 4.3. This correction is applied to the luminosity calculation each run by using the average current for the corresponding run. 
Table 4.3: Table of density correction parameters [78].

\begin{tabular}{lccc}
\hline Target & $\mathrm{p}_{0}$ & $\mathrm{p}_{1}$ & $\mathrm{p}_{2}$ \\
\hline${ }^{3} \mathrm{H}$ & $1 . \pm 0.003$ & $(-6.8 \pm 0.89) \times 10^{-3}$ & $(1.06 \pm 0.36) \times 10^{-3}$ \\
${ }^{3} \mathrm{He}$ & $1 . \pm 0.003$ & $(-5.1 \pm 0.64) \times 10^{-3}$ & $(1.04 \pm 0.25) \times 10^{-3}$ \\
${ }^{2} \mathrm{D}$ & $1 . \pm 0.003$ & $(-6.7 \pm 0.71) \times 10^{-3}$ & $(1.16 \pm 0.29) \times 10^{-3}$ \\
\hline
\end{tabular}

\subsection{Yield}

The events that pass the good electron criteria are binned in bins of $x$ with varying size to decrease statistical error for the kinematics with lower rate. The bin size ranges from 0.03 to 0.08 . Events are corrected for efficiency and background on an event by event basis. The background is accounted for through a background correction factor $\left(\mathrm{BG}_{i}\right)$. This factor is calculated to be a percentage of good events in the total sample and is applied as a multiplicative factor on an event basis. The efficiency $(\epsilon)$ factor is a combination of the efficiencies discussed in this chapter and is calculated on a run basis. The efficiencies of the current run are then applied to each good electron event.

$$
\begin{aligned}
& \text { Yield }_{\text {run }}(\text { bin })=\sum_{i}^{\text {Good Electrons(bin) }}\left(B G_{i} / \epsilon_{i}\right) \\
& \text { Yield }_{\text {kin }}(\text { bin })=\sum_{\text {run }}^{\text {runs }}\left(\text { Yield }_{\text {run }}(\text { bin })\right)
\end{aligned}
$$

The yield for a kinematic is combined on a run by run basis so that the run depended corrections can be applied to correct events for efficiencies and background. For many of the kinematics, the bins that fall within the acceptance overlap. A good electron count weighted average is used to combine the overlapping bins. Equation 4.22 describes the weighting average procedure for combining overlapping bins. Figure 4.12 shows the normalized corrected yield for ${ }^{2} \mathrm{D}$ from all kinematics. Also the combined yield is shown as a gray $x$.

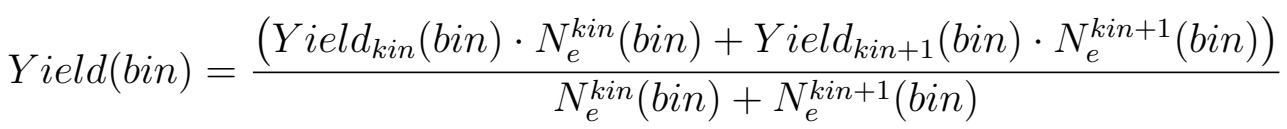




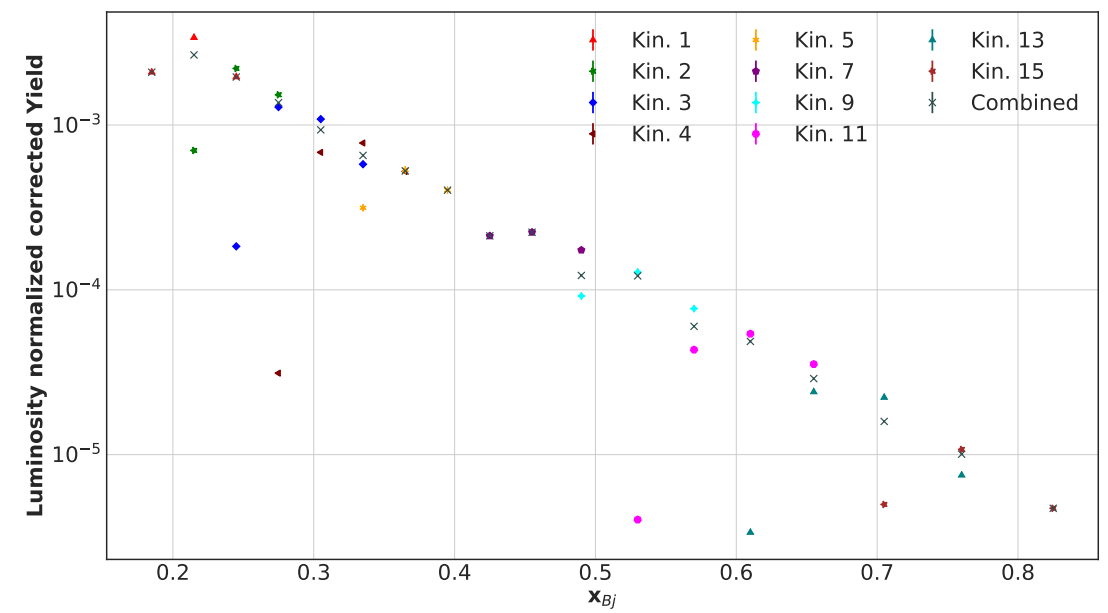

Figure 4.12: Luminosity normalized corrected yield for ${ }^{2} \mathrm{D}$. The yield for each kinematic is shown as a different color and marker, with the combined yield as a gray ' $x$ '.

\subsection{Acceptance}

The acceptance for an event in a bin of $x, \mathrm{~A}(x)$, is defined as the probability that the event will be able to pass through the spectrometer apertures and enter the VDC in a location of active detecting. The acceptance of an event is dependent on 6 variables, $\theta, \phi, \mathrm{E}^{\prime}$, and the $\mathrm{x}, \mathrm{y}$, and $\mathrm{z}$ of the reaction location. There are two ways to account for the acceptance. One is to determine an acceptance function that is dependent on all 6 of these variables. The other way is to use a Monte Carlo simulation to produce a yield that will average over all of the variables. The simulated yield can then be compared with data to account for the acceptance effects. This analysis will use a Monte Carlo simulation to account for acceptance effects via a comparison with data.

\subsection{Monte Carlo Ratio Method}

A Monte Carlo $\left({ }_{M C}\right)$ generated yield of electrons:

$$
Y_{M C}\left(E^{\prime}, \theta\right)=\mathscr{L} \cdot \sigma^{\text {model }} \cdot\left(\Delta E^{\prime} \Delta \Omega\right) \cdot A\left(E^{\prime}, \theta\right)
$$


Rewriting the Monte Carlo generated yield to emphasize the normalization factors (luminos-

ity, phase space and acceptance) allows for a direct comparison with the experimental cross section using the measured yield.

$$
\begin{aligned}
\frac{Y_{M C}(x)}{\sigma^{\text {model }}(x)} & =\mathscr{L} \cdot\left(\Delta E_{M C}^{\prime} \Delta \Omega_{M C}\right) \cdot A\left(E^{\prime}, \theta\right)_{M C} \\
\sigma^{\text {data }}(x) & =\frac{Y_{\text {data }}(x)}{\mathscr{L} \cdot\left(\Delta E_{\text {Data }}^{\prime} \Delta \Omega_{\text {Data }}\right) \cdot A\left(E^{\prime}, \theta\right)_{\text {Data }}}
\end{aligned}
$$

Using the Monte Carlo ratio method, the experimental cross section can be calculated for a bin of $x$ by:

$$
\sigma_{\text {Data }}(x)=\sigma_{\text {model }}(x) \cdot \frac{Y_{\text {Data }}(x)}{Y_{M C}(x)}
$$

This section will discuss the Monte Carlo simulation and compare the yield of Monte Carlo to the yield from data.

\subsubsection{Monte Carlo Simulation}

The process of creating MC yields contains three steps:

- production of cross section table with radiative corrections

- event generation,

- weighting accepted events with the model cross section .

\section{Cross Section Table}

A cross section model is used to weight the Monte Carlo events to make a comparison between a Monte Carlo yield and a yield from data. The Born cross section is calculated via a DIS model with an EMC model correction. An Arie Bodek model [23] is used to determine the nuclear structure function in the DIS regime. This same model uses a smearing technique to include any effects from the elastic and quasi-elastic tail. The kinematics closest to the resonance region experiences an almost negligible quasi-elastic contribution. A correction calculated from SLAC EMC data is applied to correct for the building of the nuclear structure function from free nucleon structure functions. The resulting Born cross section is used to 


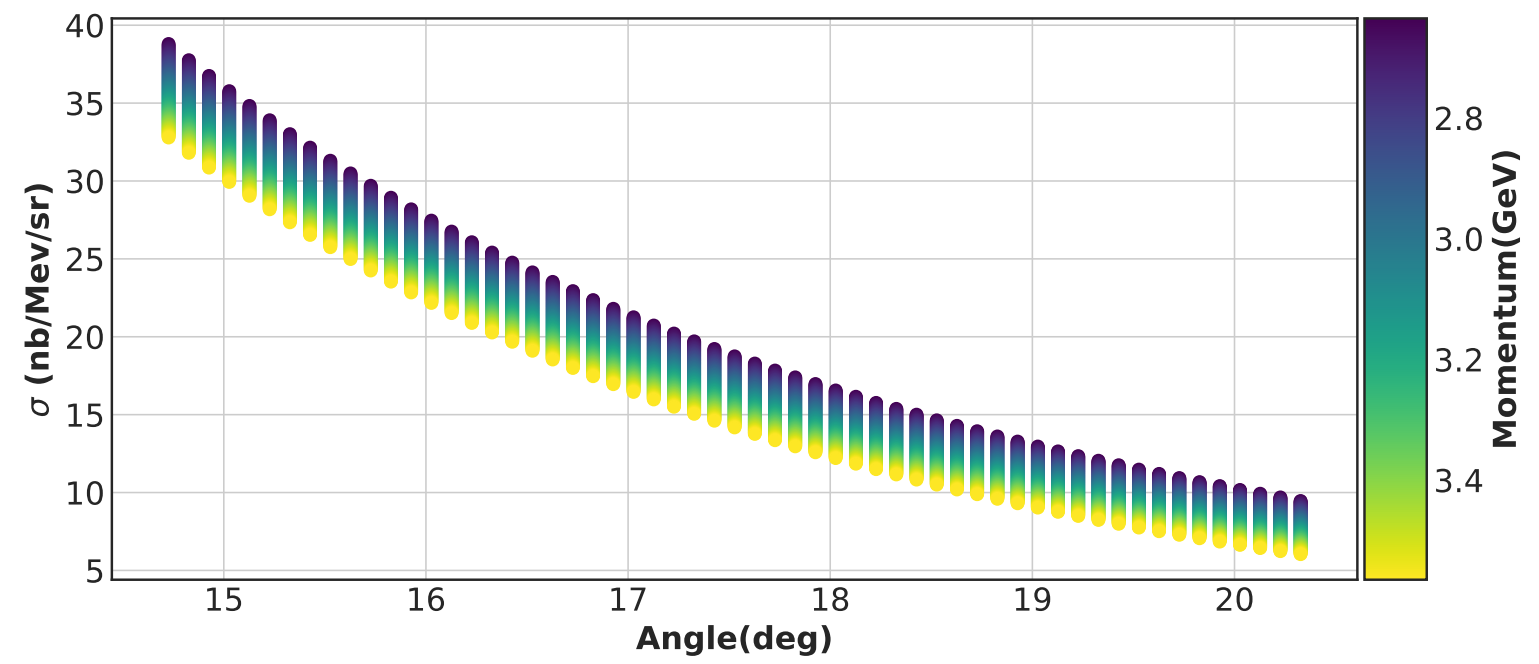

Figure 4.13: Model cross section for ${ }^{3} \mathrm{H}$ at kinematic 1 for scattered angle and momentum.

weight events in the Monte Carlo simulation. Figure 4.13 shows the born cross section for ${ }^{3} \mathrm{H}$ at kinematic one for scattered angle along the $\mathrm{x}$-axis and scattered momentum along the z-axis associated with color.

The scattered event that is measured through the experiment does not only experience the Born process (the one photon exchange approximation) shown in figure 4.14 but a combination of all the other processes shown. In order to produce a comparable result with data, these higher order contributions have to be addressed.

There are two broad types of radiative effects that needed to be accounted for during an electron nucleus scattering interaction, internal and external. External effects account for the electron radiating a real photon before or after the designated target. This radiation is caused by an interaction with fields of nuclei that make up the material other than the target. External bremsstrahlung and ionization energy losses are two of the process that occur causing these external effects. The internal radiative effects happen at the interaction point, the scattering vertex. The Feynman diagrams in figure 4.14 shows the lowest order approximation for the possible interaction channels. These high order processes are calculable in QED. Mo and Tsai [67] and S. Dasu [34] describe in detailed the method of calculating 


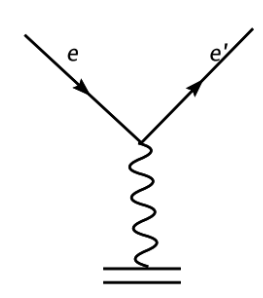

1) Born

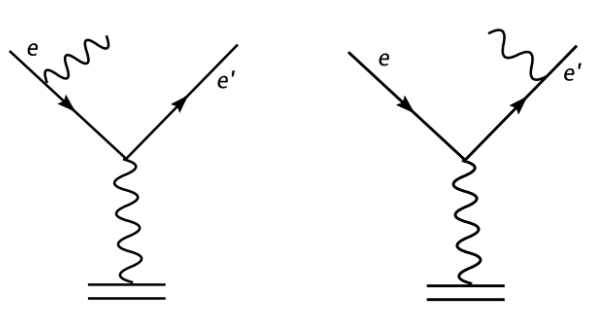

4) Bremsstrahlung

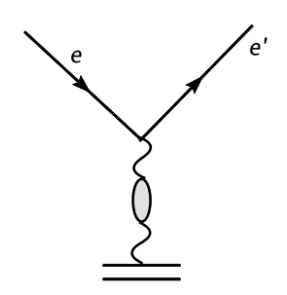

2) Vacuum polarization
3) Vertex correction

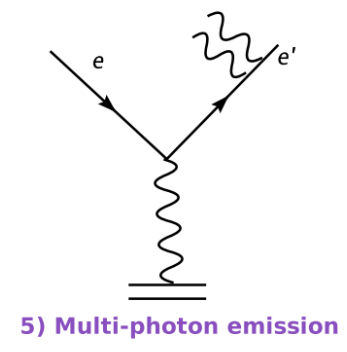

Figure 4.14: Lowest order Feynman diagrams for inclusive lepton-nucleon scattering [33].

these radiative effects. This analysis used a radiative correction code originally by S. Dasu [34]. This package uses Mo and Tsai's formula to complete the measured cross section calculation. The internal bremsstrahlung contribution is calculated via an equivalent radiator approximation. A full integral calculation is done over the entire length of radiators to make a complete calculation for the entire radiative effect [33, 34, 67, 80]. A correction is applied as a weighting factor with the Born cross section. A 2-dimensional grid is made from a scan of possible scattered angles covering the acceptance of the spectrometer for each value of scattered momentum possible for the kinematic setting. This grid is then used to populate a lookup cross section table for each target at each kinematic. The cross section and radiative correction factor are calculated for the combination of kinematic variables to complete the lookup cross section table (CST). Figure 4.15 shows the radiative correction factor $\left(R C F=\frac{\sigma_{R a d}}{\sigma_{B o r n}}\right)$ for ${ }^{3} \mathrm{H}$ at kinematic 1 for scattered angle along the $\mathrm{x}$-axis and scattered momentum along the z-axis associated with color.

\section{Generation}

The MC simulation generates events in the target frame to travel from the point of interaction to the spectrometer's focal plane. The events are generated to have a uniform 


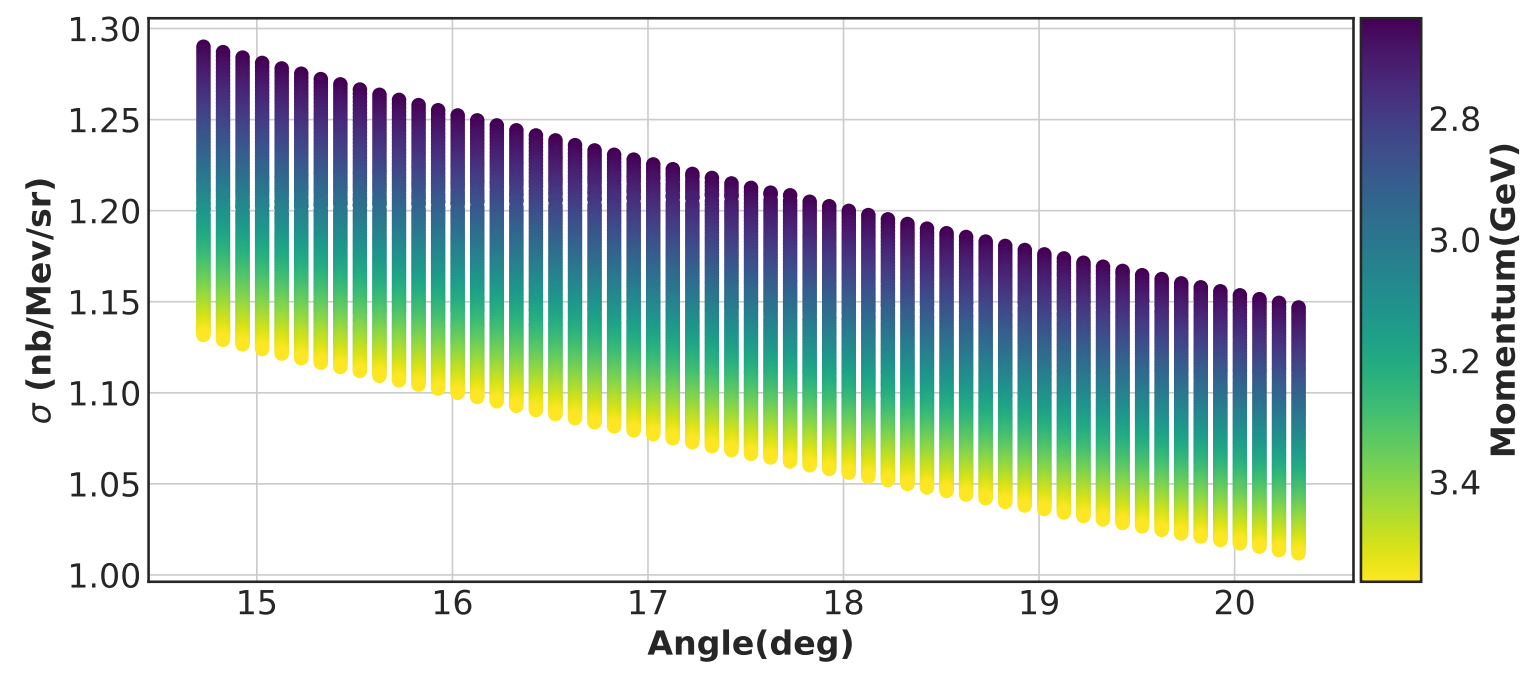

Figure 4.15: Radiative correction for ${ }^{3} \mathrm{H}$ at kinematic 1 , for scattered angle and momentum.

distribution in $\delta, \theta$, and $\phi$. A generated event drifts from the randomized point of interaction to the entrance of the spectrometer. Along this drift, the simulation calculates the chances of multiple scattering events using the radiation length of the intervening material. Simulated electrons pass through the remaining target length, aluminum end cap, vacuum, aluminum wall of the scattering chamber, air, and kapton film at the entrance of the spectrometer's vacuum. Once entering the spectrometer, the simulated event will use differential algebra based COSY model to pass through the magnetic field of the spectrometer's dipole and quadrupoles. The code checks a particle's trajectory through the spectrometer ensuring no collision with the magnetic apertures. Events that have successfully traveled to the VDC will receive a smearing effect to account for the finite resolution of the VDCs in the HRS [4]. The event's focal plane coordinates are used to transform back to the target coordinates to calculate the scattered energy and scattered angle.

\section{Event Weighting}

An accepted Monte Carlo event is weighted to best match physical conditions. The comparison of simulation with actual data requires the event to be weighted with the Born cross section of that event and corrected for radiative effects. The subroutine used 
for this analysis uses a 2 dimensional interpolation (scattered energy and momentum) to determine the Born cross section and radiative correction factor for the exact event. The 2D interpolation is completed by finding 4 locations on the lookup cross section table. For the following description of the table locations, ${ }_{e v t}=$ event and $_{c s t}=$ lookup cross section table. The cross section value is pulled from the table for a row that meets the criteria. For example, $\sigma[1]$ is the Born cross section for the row where the angle is closest but lower than the event and the momentum is closest but lower than the event.

$$
\begin{aligned}
\sigma[1] & =\sigma\left[\theta_{<}\right]\left[P_{<}\right] \rightarrow\left(P_{c s t}^{\prime}<=P_{e v t}^{\prime} \& \& \theta_{c s t}<=\theta_{e v t}\right) \\
\sigma[2] & =\sigma\left[\theta_{<}\right]\left[P_{>}\right] \rightarrow\left(P_{c s t}^{\prime}>=P_{e v t}^{\prime} \& \& \theta_{c s t}<=\theta_{e v t}\right) \\
\sigma[3] & =\sigma\left[\theta_{>}\right]\left[P_{<}\right] \rightarrow\left(P_{c s t}^{\prime}<=P_{e v t}^{\prime} \& \& \theta_{c s t}>=\theta_{e v t}\right) \\
\sigma[4] & =\sigma\left[\theta_{>}\right]\left[P_{>}\right] \rightarrow\left(P_{c s t}^{\prime}>=P_{e v t}^{\prime} \& \& \theta_{c s t}>=\theta_{e v t}\right)
\end{aligned}
$$

Each cross section value has its corresponding momentum $P[i]$, angle $\theta[i]$, and the momentum and angle differences between the event and table $\Delta P[i]$, and $\Delta \theta[i]$. The $2 \mathrm{D}$ interpolation is broken down into two 1D interpolations between the momentum values.

$$
\begin{aligned}
\sigma_{1} & =\frac{(\sigma[1] \cdot \Delta P[1]+\sigma[2] \cdot \Delta P[2])}{P[2]-P[1]} \\
\sigma_{2} & =\frac{(\sigma[3] \cdot \Delta P[3]+\sigma[4] \cdot \Delta P[4])}{P[4]-P[3]}
\end{aligned}
$$

Then an interpolation is done between the calculated $\theta$ values.

$$
\sigma=\frac{\left(\operatorname{sigma}_{2} \cdot \Delta \theta[3]+\operatorname{sigma}_{1} \cdot \Delta \theta[1]\right)}{\theta[3]-\theta[1]}
$$

The 2D interpolation is completed both for the Born cross section and the radiative correction factor for each accepted simulated event. 

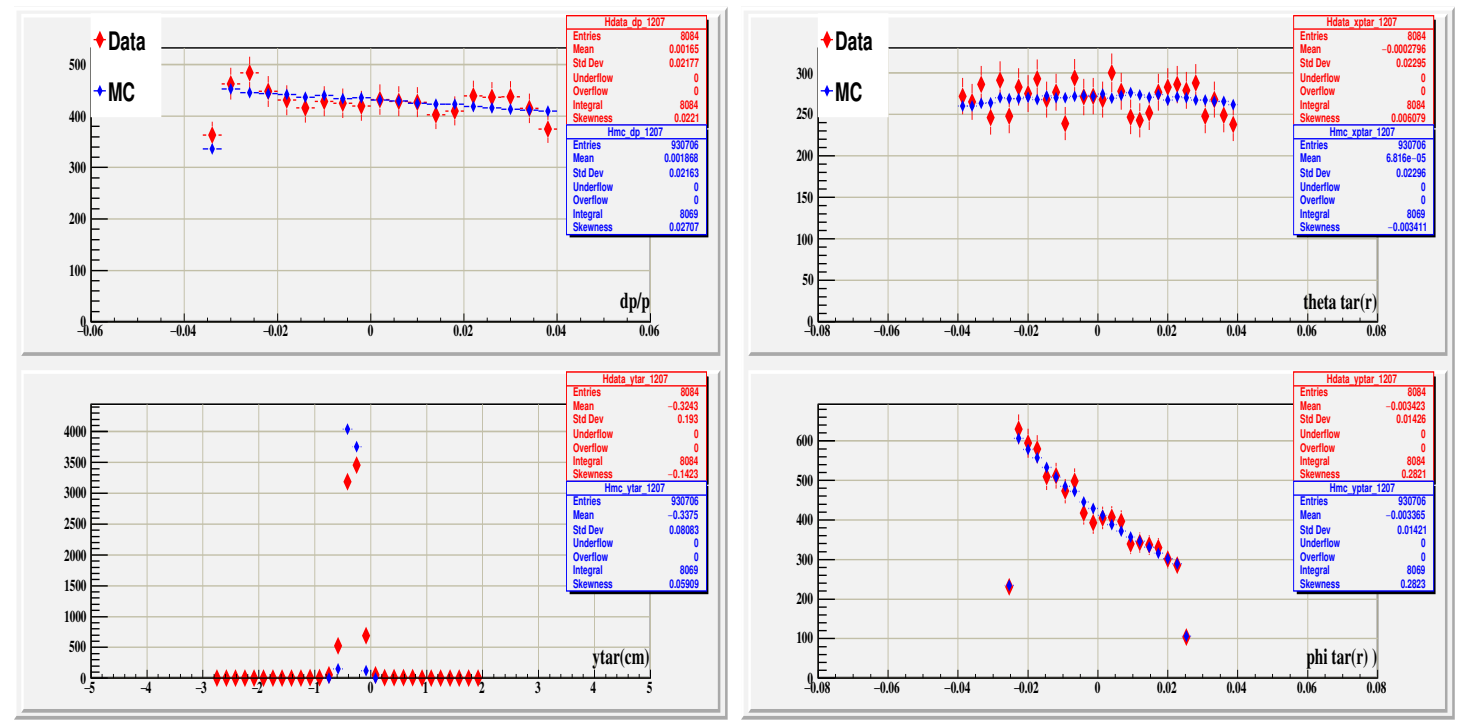

Figure 4.16: Monte Carlo to Data comparison for target plane variables, top left $\delta$,bottom left $\mathrm{y}$, top right $\theta$, bottom right $\phi$. Run 1207, kinematic 1 on carbon foil.

\subsubsection{Monte Carlo Comparison}

The weighted Monte Carlo events are binned into the target and focal planes for comparison to data. The comparison is used to determine the quality of the simulation. Figure 4.16 and 4.17 show the comparison for target plane variables and focal plane variables respectively. The important target variables to study are $\delta_{t g}, \mathrm{y}_{t g}$, out of plane angle $\left(\theta_{t g}\right)$, and the in plane angle $\left(\phi_{t g}\right)$. The yield dependence in $\phi_{t g}$ and $\delta_{t g}$ are expected due to the dependence of the cross section on those variables. The good comparison for those quantities show the simulation's good performance in matching the spectrometers acceptance and the cross section models functional form. The comparison between data and Monte Carlo has been deemed good, with $99 \%$ comparison for the single foil carbon target. I show the comparison between data and Monte Carlo in bins of $x$ for cross section extraction in figure 4.18. This ratio factor will be applied to the model cross section to extract the measured cross section. I will discuss the cross section results and their uncertainties in the next section. In appendix B, I discuss the beginning stages of a simulation I created to study the dependence of nucleon momentum on the EMC effect. 

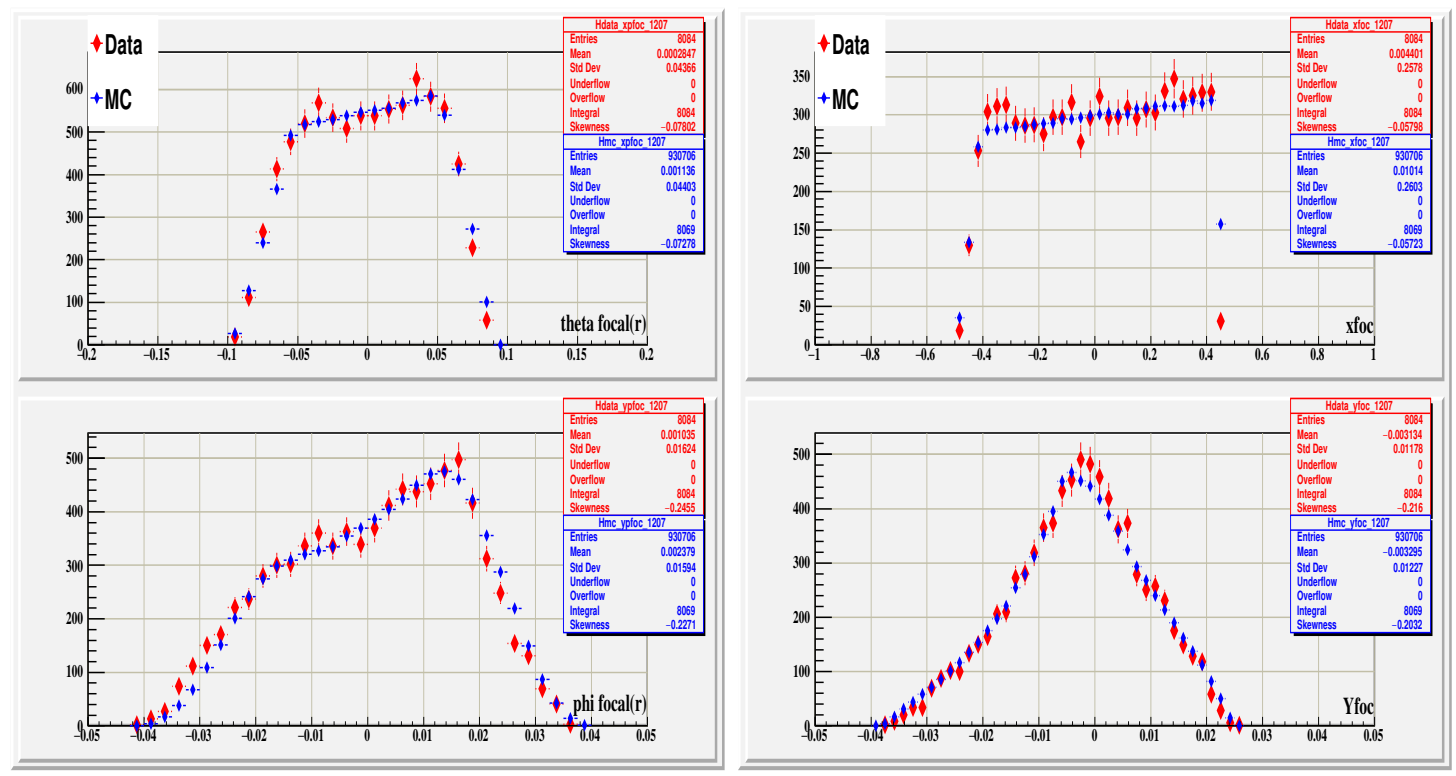

Figure 4.17: Monte Carlo to Data comparison for focal plane variables, top left $\theta$,bottom left $\phi$, top right $x$, bottom right $y$. Run 1207, kinematic 1 on carbon foil.

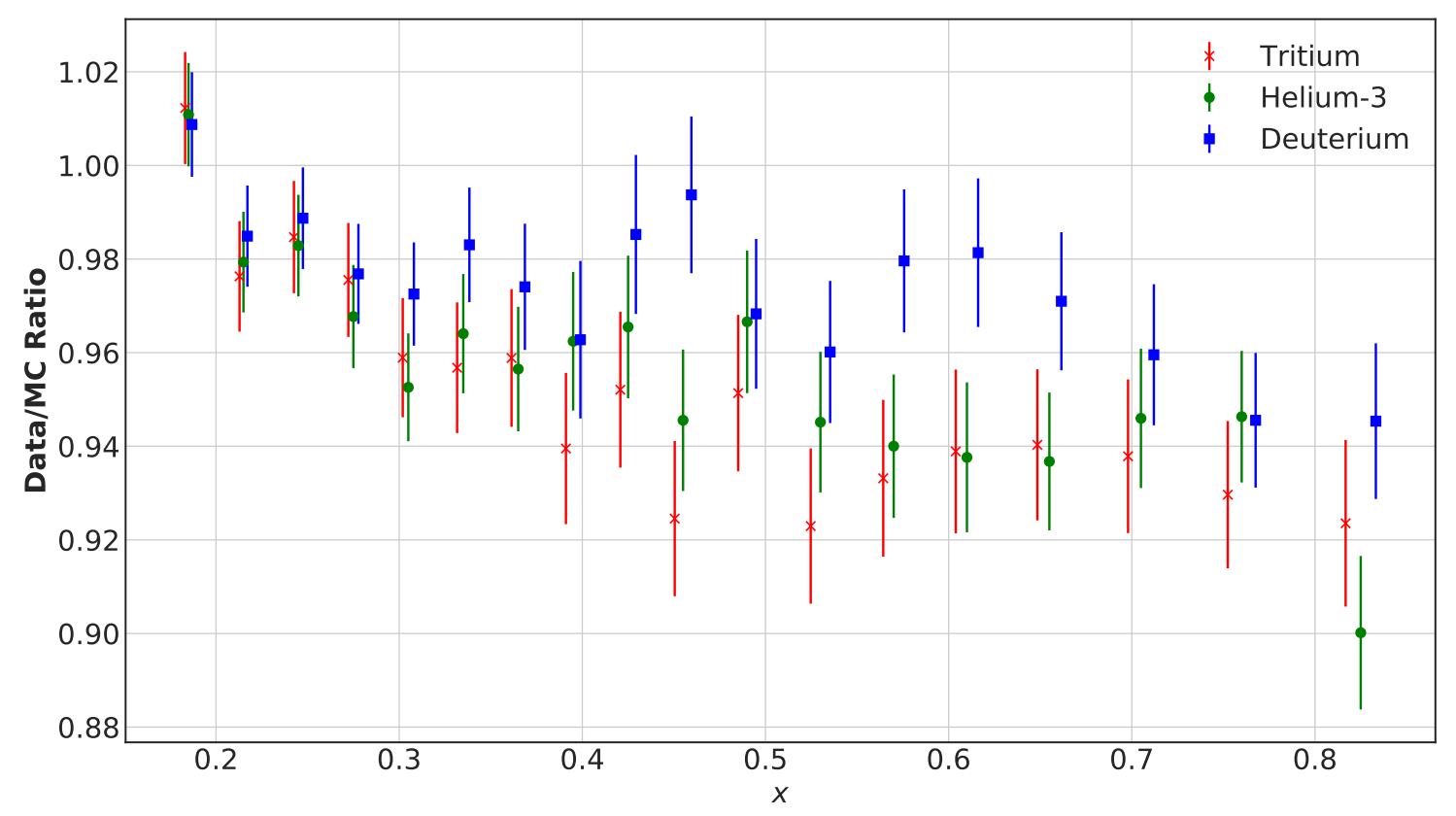

Figure 4.18: The ratio of data to Monte Carlo yield in bins of $x$ for three gas targets. 


\section{Chapter 5}

\section{Results}

The culminating part of this nuclear physics analysis begins with the extraction of the experimentally measured DIS cross sections for three targets. Then using those cross sections to study the per nucleon scaled A/D ratio. I will also use these A/D ratios to study the EMC effect for both ${ }^{3} \mathrm{He}$ and ${ }^{3} \mathrm{H}$. In this chapter, I will present my results for the DIS cross sections and EMC effect. I will also discuss an error analysis for both the cross section measurements and the EMC effect results.

\subsection{DIS Cross Section}

Using the Monte Carlo ratio method, I extracted the experimental measured cross section for ${ }^{3} \mathrm{He},{ }^{3} \mathrm{H}$, and D. These DIS cross section extraction ranges from 0.18 to 0.82 in $x$, from 2.2 to $11.8 \mathrm{GeV}^{2}$ in $Q^{2}$, and has $\mathrm{W}^{2}>3.5 \mathrm{GeV}^{2}$. The central momentum setting of the spectrometer was $3.1 \mathrm{GeV} / \mathrm{c}$ and the spectrometer was moved from 17.5 to 33.5 degrees. Normalization uncertainty due to target thickness uncertainty for ${ }^{3} \mathrm{H}=0.97 \%,{ }^{3} \mathrm{He}=1.12 \%$, and $\mathrm{D}=0.56 \%$.

$$
\sigma_{\text {Data }}=\sigma_{\text {model }} \cdot \frac{Y_{\text {Data }}}{Y_{M C}} .
$$

I compared the data yield to the Monte Carlo yield to produce a correction factor for the model cross section. Figure 4.18 shows the ratio comparison between data and Monte Carlo 


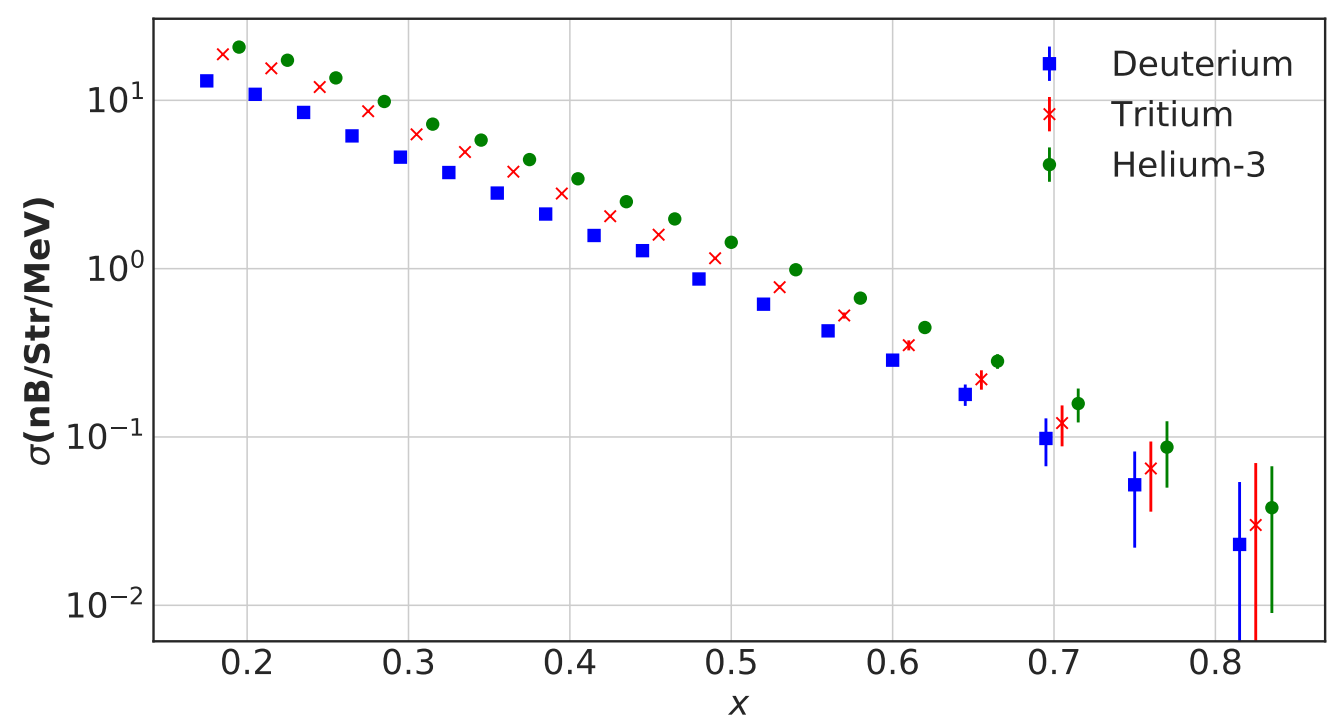

Figure 5.1: Experimentally measured cross section using the Monte Carlo ratio method for ${ }^{3} \mathrm{H},{ }^{3} \mathrm{He}$, and D.

in bins of $x$. I apply this Monte Carlo to data ratio factor to the model cross section to extract out the experimentally measured cross section for a bin of $x$. Figure 5.1 shows a plot of the experimentally measured cross section for ${ }^{3} \mathrm{H},{ }^{3} \mathrm{He}$, and D. This is the first result of an inclusive DIS cross section for ${ }^{3} \mathrm{H}$ and expands on the DIS cross section for ${ }^{3} \mathrm{He}$, pushing the invariant mass past the resonance region at higher values of $x$. Appendix A contains tables of the cross section and summaries of the errors included in the error study for the cross section analysis of each target.

\subsection{Cross Section Error Analysis}

The measurement of the cross section requires finely tune detectors and analysis procedures. Uncertainties arise due to the nature and limitations of the spectrometers and the analysis of the data received. In this section, I will discuss the calculation and propagation of errors in the analysis of the DIS cross section. The error analysis will be broken into the calculation of normalized yield for data, yield for Monte Carlo, and the model used for cross section extraction. Table 5.1 contains the summary of the errors for the cross section extraction for the error on the yield, $\mathrm{MC}$, and model. 
Table 5.1: Relative Error Contributions for Cross Section for a selection of bins. This table contains the summary of relative error to the cross section for the yield, Monte Carlo, and Cross Section Model. Then summed in quadrature is the total relative error for the cross section.

\begin{tabular}{|l|l|l|l|l|l|l|}
\hline Xbjc & $\mathbf{0 . 1 8 5}$ & $\mathbf{0 . 3 0 5}$ & $\mathbf{0 . 4 9}$ & $\mathbf{0 . 5 7}$ & $\mathbf{0 . 7 0 5}$ & $\mathbf{0 . 8 2 5}$ \\
\hline Corrected Norm. Yield Error & 0.01 & 0.01 & 0.014 & 0.015 & 0.014 & 0.016 \\
\hline MC and Model Total Error & 0.015 & 0.015 & 0.013 & 0.014 & 0.028 & 0.026 \\
\hline Cross Section Error & 0.018 & 0.018 & 0.019 & 0.021 & 0.031 & 0.031 \\
\hline
\end{tabular}

\subsubsection{Normalized Yield Error}

\section{Corrected Electron Count Error}

$$
\begin{aligned}
\frac{d \sigma}{d E^{\prime} d \Omega} & =\frac{(N-B G)}{\mathscr{L} \cdot \epsilon \cdot \Delta E^{\prime} \Delta \Omega \cdot A\left(E^{\prime}, \theta\right)} . \\
\sigma & =\text { NormY } /\left(\Delta E^{\prime} \Delta \Omega \cdot A\left(E^{\prime}, \theta\right)\right) \\
\text { NormY } & =\left(N_{e} \cdot B G / \epsilon\right) / \mathscr{L}
\end{aligned}
$$

The contributions for error on the measurement of the normalized yield(normY) can be simplified into the contributions for the corrected number of electrons and the luminosity. The error for the corrected number of counted electrons includes the random statistical error for the count of electrons detected, the error associated with the calculated efficiencies, and the error from the background correction. The electron statics range from 4875 to more than 42000 in a bin and therefore I have treated the randomness of the counting in a standard error technique. The relative statistical error for a bin is $\frac{1}{\sqrt{N e}}$ and ranges from $.5 \%$ to $1.4 \%$. Calculating the efficiencies deals with calculating a probability of a binomial choice. The errors associated with the efficiencies are calculated using the Wilson score interval described in equation 5.3, where $p$ is the efficiency or a probability of a success, $n$ is the number of samples, $z$ is 1.645 for a $95 \%$ confidence level.

$$
p\left[\left[_{\text {UpperBound }, \text { LowerBound }}\right]=\frac{2 n p+z^{2} \pm\left[z \sqrt{z^{2}-\frac{1}{n}+4 n p(1-p)+(4 p-2)}+1\right]}{2\left(n+z^{2}\right)}\right.
$$


The relative efficiency errors are added in quadrature to calculate an over all efficiency error to be propagated into the yield calculation.

The correction for background events corrects for events from the end caps and pair produced electrons. The end cap correction factor is produced by a ratio of yields between the gas cells and the empty cell. This yield measurement allows for the cancellation of many of the systematic uncertainties. The largest contribution of this error is the low statistics of the background events. The estimated error to the cross section analysis is about $1 \%$. This estimated is derived by associating the uncertainty to a random statical fluctuation. Further study of the end cap contamination and the error associated with this correction is part of the ongoing analysis. The error for the positron correction involves using the covariance matrix for the exponential fit and propagating the error for each parameter of each target using the $x_{B j}$ dependent function. The error for the fit parameters are propagated to an error for the correction using equation 5.4.

$$
\frac{\Delta f(x)}{f(x)}=\sqrt{\sum_{i}\left(\delta p_{i} \cdot x^{i}\right)^{2}+\sum_{i j}\left(2 \cdot \delta p_{i j} \cdot \frac{d f(x)}{d p_{i}} \cdot \frac{d f(x)}{d p_{i}}\right)}
$$

The error for the background corrections are added in quadrature to get an overall error for the total back ground correction.

\section{Luminosity Error}

The errors involved in the luminosity calculation are the target density thickness measurement, beam charge calculation, and density correction. The error in the target thickness measurement is considered an overall normalization uncertainty. The error for the three gas targets' thickness measurements are: ${ }^{3} \mathrm{H}=0.97 \%,{ }^{3} \mathrm{He}=1.12 \%$, and $\mathrm{D}=$ $0.56 \%$ [66]. The measurement uncertainties for each gas target are displayed in table 4.2. The amount of beam charge deposited on the target is calculated via the BCM (beam charge monitors) and their calibrations. The BCM calibrations were determined a few times

through the run period with no change to the calibration constants. Due to the consistency of the BCMs and the high quality calibration the error estimation for charge calculation 
Table 5.2: This table provides a summary of the error analysis for the Corrected Normalized Electron Yield. Containing the relative error for the bin statistics, background subtraction, efficiencies, and luminosity calculation. Including the total error on the Yield analysis for a selection of bins over the entire range of kinematics.

\begin{tabular}{|c|c|c|c|c|c|c|}
\hline Xbjc & 0.185 & 0.305 & 0.49 & 0.57 & 0.705 & 0.825 \\
\hline Stat Err & 0.0062 & 0.0053 & 0.0097 & 0.0106 & 0.011 & 0.0143 \\
\hline Density Correction & 0.002 & 0.0024 & 0.003 & 0.003 & 0.0026 & 0.002 \\
\hline Positron Correction & 0.0004 & 0.0002 & 0.0001 & 0.0001 & 0.0001 & 0.0001 \\
\hline End Cap Subtraction & 0.007 & 0.007 & 0.007 & 0.007 & 0.0070 & 0.007 \\
\hline Efficiencies Error & 0.004 & 0.0051 & 0.0075 & 0.0071 & 0.0041 & 0.0032 \\
\hline Corrected Norm. Yield Error & 0.0104 & 0.0104 & 0.0145 & 0.0148 & 0.0139 & 0.0164 \\
\hline
\end{tabular}

is approximately $0.2 \%$. The error for the density correction is based in the error from the 2nd order polynomial fit. The error is calculated using the same method as the positron background correction, combining the errors for the fit parameters and the covariance terms through the propagation formula 5.4 using the current dependent correction function, 4.20. The relative error in charge on target and density correction have been added in quadrature to calculate a systematic error for the luminosity calculation. The systematic error for the luminosity and the systematic error for the correct count of electrons are then added in quadrature to calculate an overall normalized yield error.

\subsubsection{Monte Carlo Yield Error}

The responsibility of the Monte Carlo is to accurately account for the acceptance of the spectrometers. The uncertainty for the Monte Carlo yield combines the statical uncertainty of the number of events generated and the reconstruction of the momentum and angle. The statistical uncertainty is reduced due to the generation of a few million events. The uncertainty in the reconstruction of an event is a mixture of the uncertainties in the beam $\mathrm{x}$ and $\mathrm{y}$ position, spectrometer offsets, beam energy, optics, and acceptance cuts. The uncertainties for the beam $\mathrm{x}$ and $\mathrm{y}$ position were controlled by the BPMs and the calibration I completed. The uncertainty for the BPM measurements is $140 \mu \mathrm{m}$. The beam energy measurement is quoted to have an absolute accuracy of $5 \times 10^{-4}$. The optics are capable of determining the relative in-plane angle with an accuracy of $\pm 0.2 \mathrm{mrad}$, out-of-plane 
angle with $\pm 0.6 \mathrm{mrad}$ in the target coordinates, and momentum reconstruction resolution $2.5 \times 10^{-4}[4]$. Using equation 4.8, I propagated the resolution of target reconstruction variables to determine the relative uncertainty of the scattered angle to be $\approx 0.15 \%$. I have calculated the effect of the accuracy of the optics by comparing the cross section for the nominal values of beam energy, scattered angle, and momentum, to the cross section of these values shifted by their uncertainties. A summary of the uncertainties used in the uncertainty of optics reconstruction are listed below. In table 5.3, I summarize the relative errors associated the Monte Carlo yield and the model cross section discussed in the next section.

- $\delta \theta=0.15 \%$

- $\delta E^{\prime}= \pm 0.025 \%$

- $\delta E_{\text {beam }}= \pm 0.05 \%$

Using the errors in scattering angle, scattering momentum, and beam energy, I calculated an approximate error associated with the reconstruction events into $x$ bins with equation 5.5. The fluctuation of this error is large and ranges from $0.5 \%$ to $1.5 \%$ and therefore will be applied on a point to point basis.

$$
\frac{\Delta \sigma\left(E, E^{\prime}, \theta\right)}{\sigma\left(E, E^{\prime}, \theta\right)}=\frac{\sigma\left(E+\Delta E_{\text {beam }}, E^{\prime}+\Delta E^{\prime}, \theta+\Delta \theta\right)-\sigma\left(E, E^{\prime}, \theta\right)}{\sigma\left(E, E^{\prime}, \theta\right)}
$$

\subsubsection{Cross Section Model Error}

The model dependence of the DIS cross section and the radiative corrections are the two factors of the systematic error that accompanies the Monte Carlo ratio method. My analysis of the model dependent errors used different DIS cross section models to supply an error for the model cross section and the radiative corrections. The models I will uses to study the model dependent error can be broken into 3 sections, models for $\mathrm{F}_{2 d}$, model for EMC correction, and model for the ratio for $F_{2 n} / F_{2 p}$. I will use two models to form the D structure function, Ari Bodek [23] and M. Arneodo's NMC model [7]. I will use two EMC correction models, Kulagin, S. A. and Petti, R. [59], and a SLAC EMC correction [22]. The structure function ratio is formed by two models also, linear Ratio $=(1-0.8 \times x)$ and NMC [71]. The 
Table 5.3: This table contains a summary of the relative error for the Monte Carlo yield extraction and the model cross section. Also, I include the errors add in quadrature for these two analysis parts.

\begin{tabular}{|l|l|l|l|l|l|l|}
\hline \multicolumn{1}{|r|}{ Xbjc } & $\mathbf{0 . 1 8 5}$ & $\mathbf{0 . 3 0 5}$ & $\mathbf{0 . 4 9}$ & $\mathbf{0 . 5 3}$ & $\mathbf{0 . 7 0 5}$ & $\mathbf{0 . 8 2 5}$ \\
\hline MC Stat Error & 0.003 & 0.002 & 0.003 & 0.002 & 0.002 & 0.002 \\
\hline Resolution Error & 0.015 & 0.011 & 0.006 & 0.004 & 0.007 & 0.02 \\
\hline Model Error & 0.002 & 0.01 & 0.011 & 0.011 & 0.027 & 0.018 \\
\hline MC and Model Total Error & 0.015 & 0.015 & 0.013 & 0.012 & 0.028 & 0.026 \\
\hline
\end{tabular}

model depended error was estimated by comparing the different models together with the chosen model. In the extreme case, this error reached a maximum of $2 \%$.

\subsection{EMC Ratios}

In this section, I will present the A/D cross section ratios for ${ }^{3} \mathrm{He}$ and ${ }^{3} \mathrm{H}$. The ratios are normalized by the number of nucleons in each target. Included in figure 5.2 are results from E03103. These results have different invariant mass but are considered DIS until 0.65 in $x$. Above this threshold, the invariant mass begins to drop lower then the DIS criteria used for my analysis.

\subsubsection{Isoscalar Correction}

The definition of an EMC effect ratio is the ratio of the per nucleon cross section ratio of a target nucleus with D. Also, this comparison is of a target with equal number of protons and neutrons, an isoscalar nuclei. When looking at the EMC ratio of a target with an unequal number of nucleons, an isoscalar correction needs to be applied. This correction is used to correct for the difference in cross section between the neutron and proton. The per nucleon nuclear $\mathrm{F}_{2}^{A}$ of an unsymmetrical nucleus can be written as:

$$
\frac{1}{A}\left(Z F_{2}^{p}+(A-Z) F_{n}^{2}\right)
$$




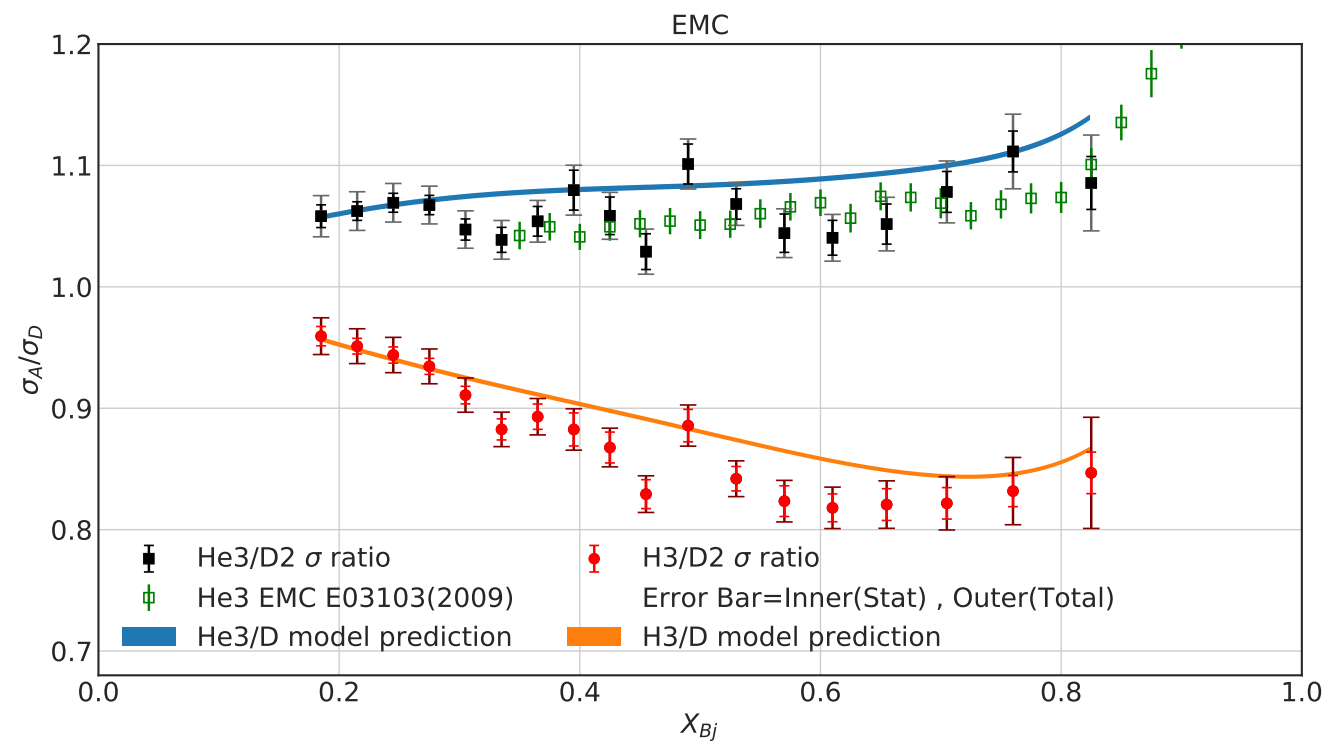

Figure 5.2: The A/D ratio for ${ }^{3} \mathrm{He}$ and ${ }^{3} \mathrm{H}$ from my analysis. Also included, EMC analysis from E03103[79] and the EMC ratios from a DIS scattering model from Arie Bodek model [23].

and a symmetrical nucleus can be simplified to:

$$
\frac{1}{2}\left(F_{2}^{p}+F_{n}^{2}\right) .
$$

The isoscalar correction can be determined by applying a correction function $f_{\text {iso }}$ to equate the two previous equations and solving that combination for the correction function.

$$
\begin{gathered}
\frac{1}{2}\left(F_{2}^{p}+F_{n}^{2}\right)=f_{\text {iso }}^{A} \frac{1}{A}\left(Z F_{2}^{p}+(A-Z) F_{n}^{2}\right) \\
f_{i s o}^{A}=\frac{\frac{1}{2}\left(1+F_{2}^{n} / F_{n}^{p}\right)}{\frac{1}{A}\left(Z+(A-Z) F_{2}^{n} / F_{n}^{p}\right)}
\end{gathered}
$$

The isoscalar correction for a nuclear target depends on $\mathrm{A}, \mathrm{Z}$, and the $\mathrm{F}_{2}$ ratio. There exists no data on the free neutron, so the $F_{2}^{n} / F_{n}^{p}$ is a model dependent extraction. The $\mathrm{F}_{2}$ ratio has been extracted from the ratio of ${ }^{3} \mathrm{H}$ to ${ }^{3} \mathrm{He}$ from the MARATHON experiment and D to hydrogen ratios from previous experiments from SLAC and the NMC. I have used the $F_{2}^{n} / F_{n}^{p}$ from MARATHON's ${ }^{3} \mathrm{H}$ to ${ }^{3} \mathrm{He}$ ratio to calculate my isoscalar correction for both ${ }^{3} \mathrm{He}$ and ${ }^{3} \mathrm{H}$. I have estimated the error of this model dependent correction by comparing 
the isoscalar correction from the MARATHON extraction with the isoscalar correction using three other $F_{2}^{n} / F_{n}^{p}$ models Kulagin, S. A. and Petti, R. [59], J. Arrington [8], and NMC [71]. The relative variance of this calculation ranges from $\pm 0.1 \%$ to $\pm 3.8 \%$ for ${ }^{3} \mathrm{H}$ EMC ratio. These 4 models and the error band from this study are shown in figure 5.3 for the isoscalar correction on ${ }^{3} \mathrm{H}$ and figure 5.4 for the isoscalar correction on ${ }^{3} \mathrm{He}$.

\subsection{EMC Effect}

Using the extracted cross section of the two $A=3$ nuclei and $D$, the first measurement of

the ${ }^{3} \mathrm{H}$ EMC effect has been achieved. The data also provides a comparison of the EMC effect of the mirror nuclei ${ }^{3} \mathrm{He}$. MARATHON's design of rotating targets, and uniform data taking procedure allows for the cancellation of the detector efficiency correction factors and corrections due to acceptance issues. This will reduce the size of the uncertainty of the EMC effect measurement and provides precise result for the EMC ratio of the two $\mathrm{A}=3$ nuclei.

The isoscalar corrected EMC Effect ratios for ${ }^{3} \mathrm{He}$ and ${ }^{3} \mathrm{H}$ from my inclusive DIS cross section extraction are displayed in figures 5.5 and 5.6. These results provide a comparison between my extraction of the ${ }^{3} \mathrm{He}$ EMC and the ${ }^{3} \mathrm{He}$ EMC results from the E03103 from J. Seely and A. Daniels [79]. My EMC effect for ${ }^{3} \mathrm{He}$ shows good agreement with the E03103 experimental results within uncertainty. The EMC results are plotted with error bars that represent the total point to point uncertainty for the EMC measurement and an error band for the overall normalization uncertainty dominated by the error in target thickness measurement.

\subsubsection{Ratio of EMC Effects}

If figure 5.7, I display the results of the ratio of the two $\mathrm{A}=3$ mirror nuclei EMC effects. The errors associated with the extraction of this ratio are the errors for extracting both cross sections and the errors for the ratio of the isoscalar correction. For the errors on the isoscalar correction, I looked at the ratio of isoscalar correction for the different models and extracted 


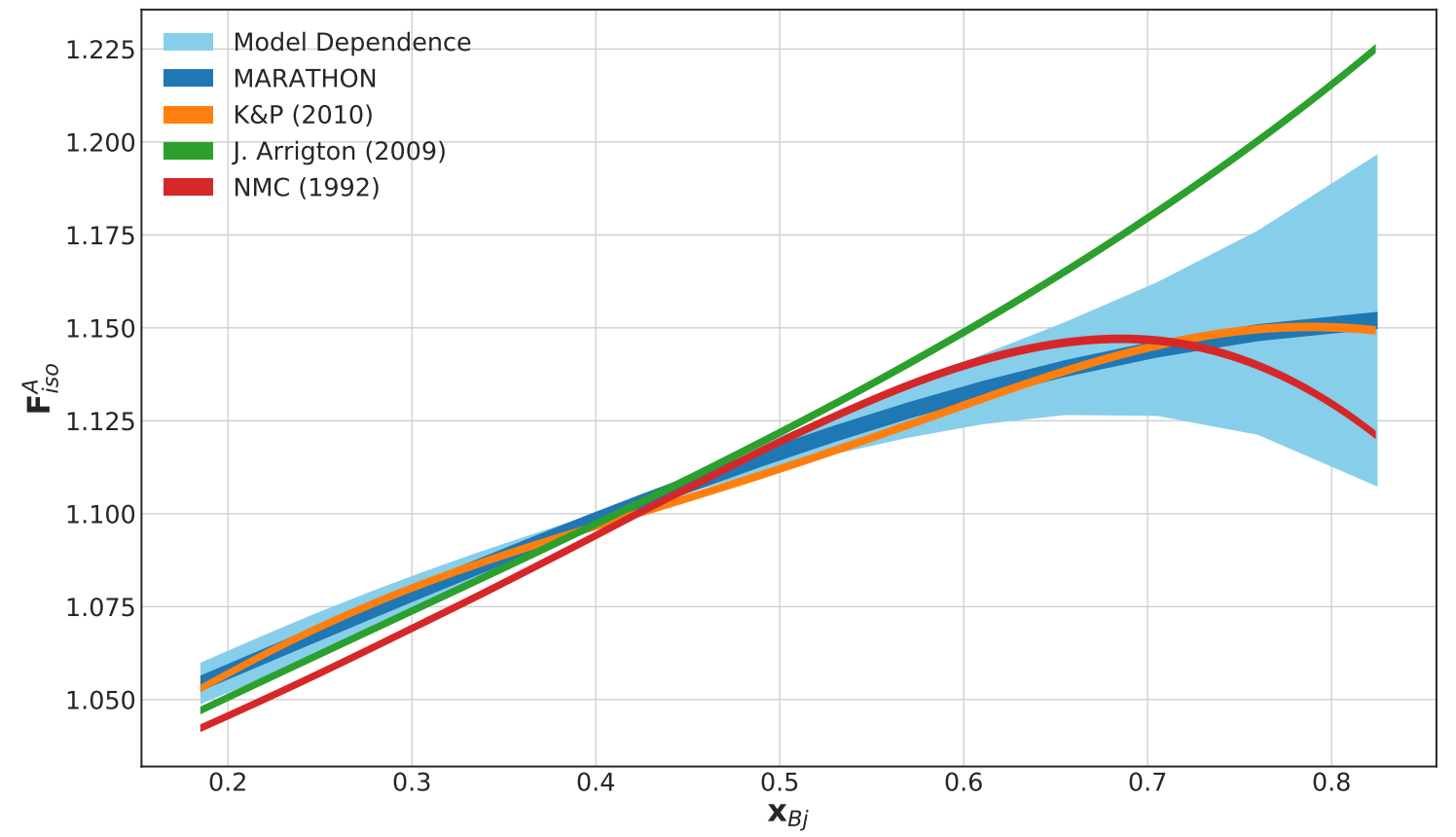

Figure 5.3: Isoscalar correction factor for the ${ }^{3} \mathrm{H}$ EMC ratio from four different models discussed in this section.

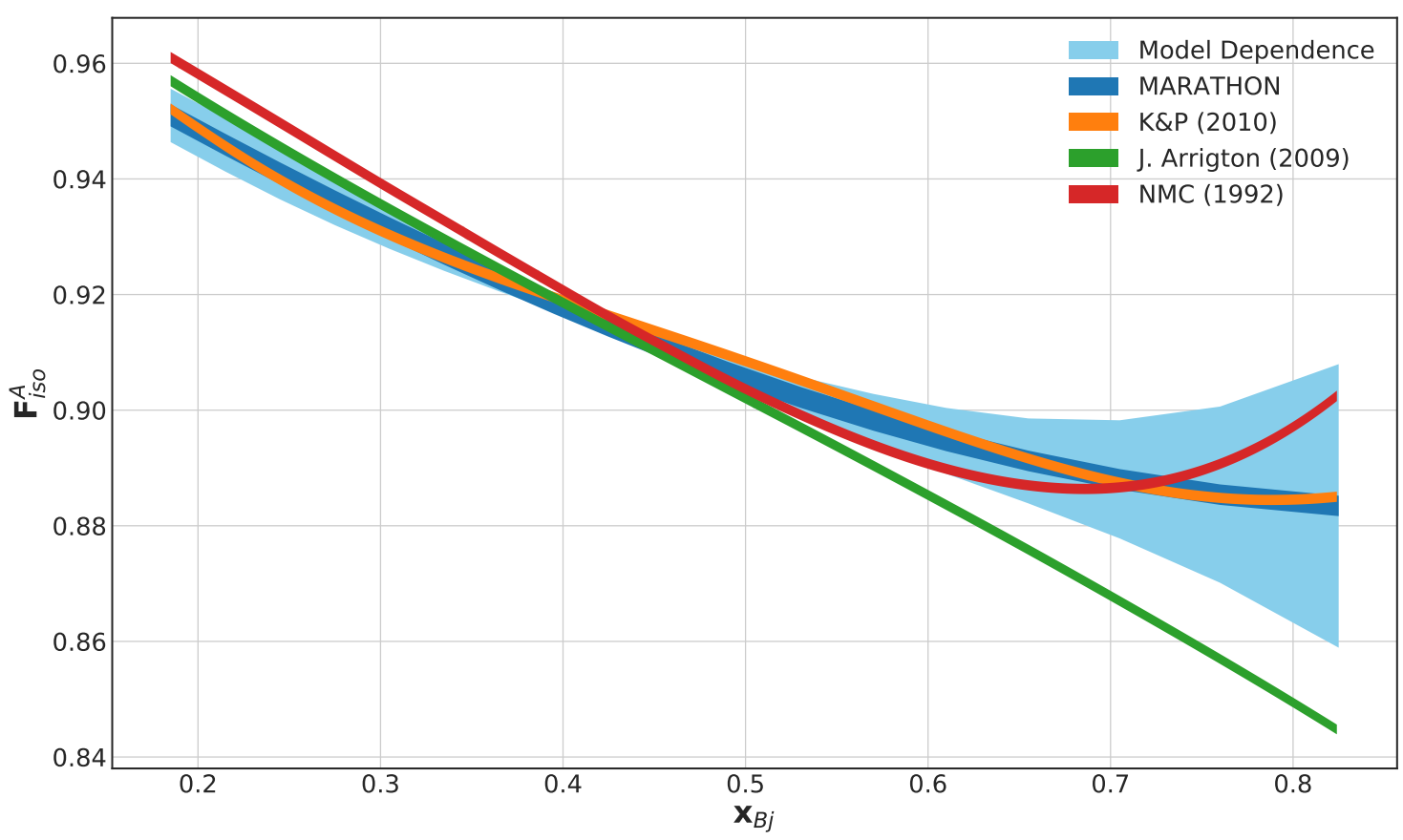

Figure 5.4: Isoscalar correction factor for the ${ }^{3} \mathrm{He}$ EMC ratio from four different models discussed in this section. 


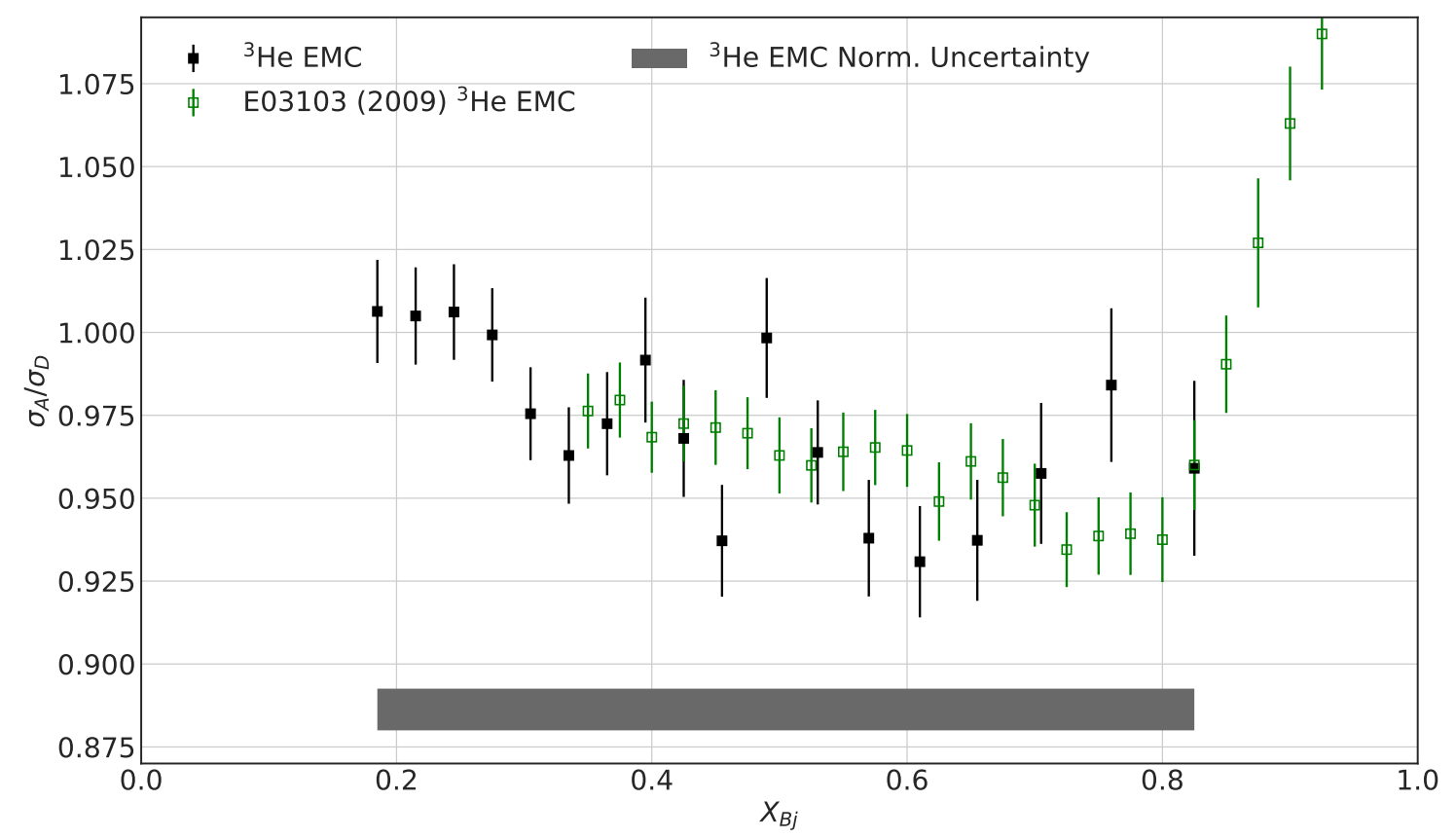

Figure 5.5: Isoscalar corrected EMC ratio for ${ }^{3} \mathrm{He}$. The point to point error bars are the total systemic and statical errors for the EMC effect.

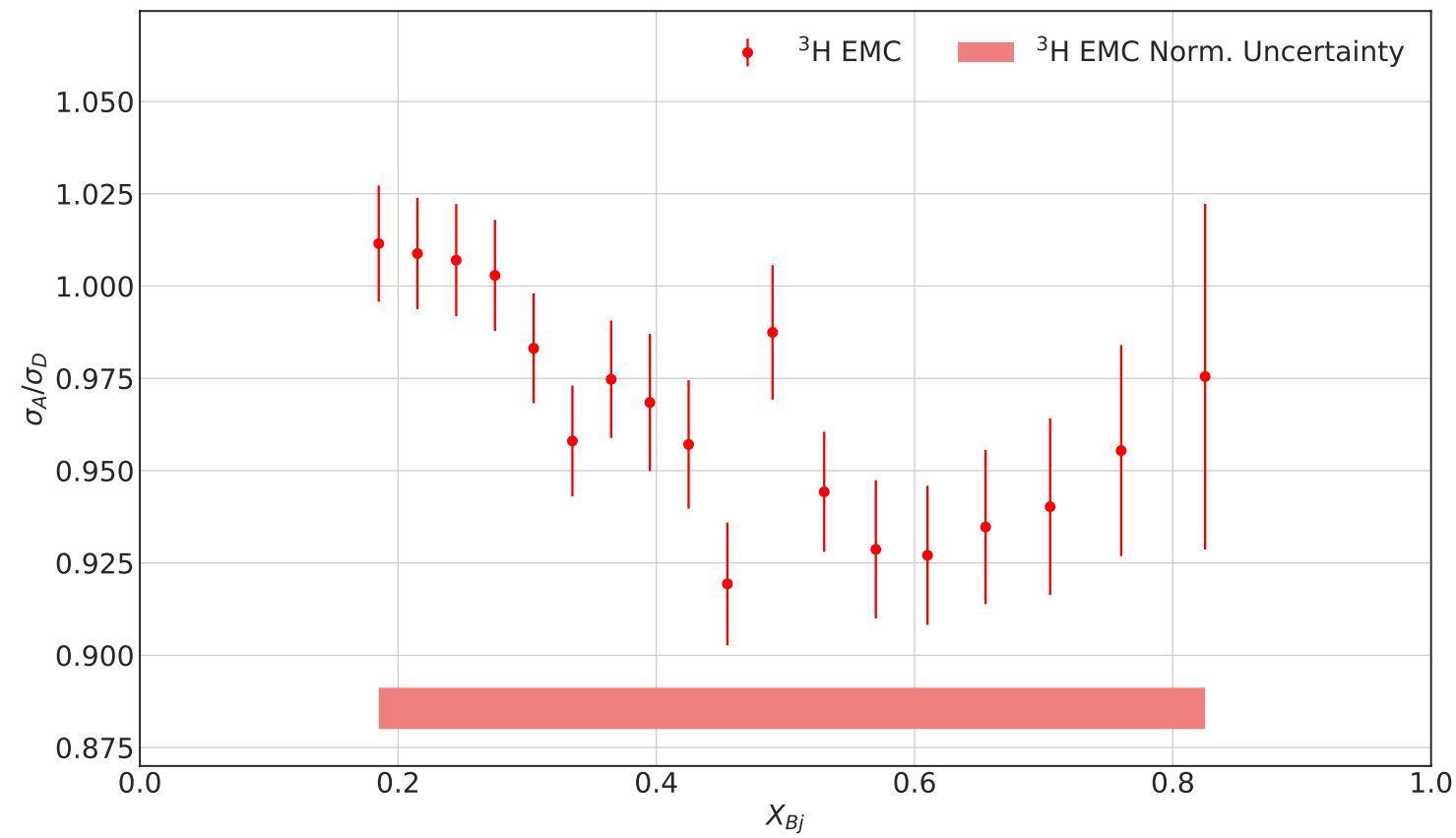

Figure 5.6: Isoscalar corrected EMC ratio for ${ }^{3} \mathrm{H}$. The point to point error bars are the total systemic and statical errors for the EMC effect. 


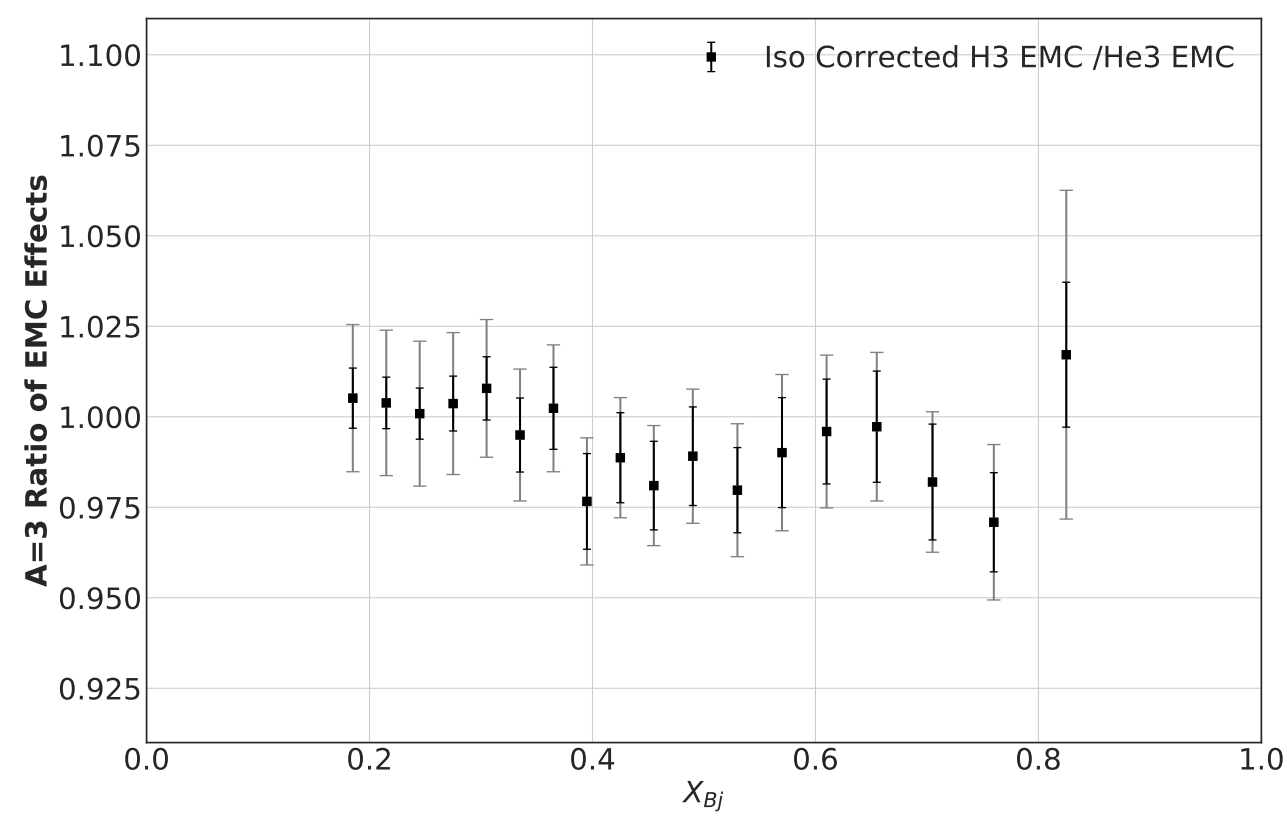

Figure 5.7: Ratio of the isoscalar corrected ${ }^{3} \mathrm{H}$ EMC Effect and ${ }^{3} \mathrm{He}$ EMC Effect. The error bar is statistical error only, while the band includes statical and systematic errors.

the standard deviation from the model dependence on the ratio. The error bars in figure 5.7 are all error contributions added in quadrature. 


\section{Chapter 6}

\section{Conclusion}

The MARATHON experiment took inclusive DIS data on the specially designed sealed ${ }^{3} \mathrm{H}$ cells, filled with ${ }^{3} \mathrm{H},{ }^{3} \mathrm{He}$, and ${ }^{2} \mathrm{D}$ at the Thomas Jefferson National Accelerator Facility. The unique opportunity provided by access to ${ }^{3} \mathrm{H}$ allowed the MARATHON experiment to be the first to use DIS to study the internal structure of this radioactive $A=3$ system. Alongside ${ }^{3} \mathrm{H}$, DIS data were taken on both ${ }^{3} \mathrm{He}$ and ${ }^{2} \mathrm{D}$ for ratio comparison. The experimental kinematics produces DIS events for $x$ from 0.18 to 0.8 , with a $\mathrm{Q}^{2}$ range of 3 to $12\left(\mathrm{GeV}^{2}\right)$ by rotating the electron spectrometer from 17.5 to 36 degrees and using the Continuous Electron Beam Accelerator Facility's 10.6 GeV beam.

The MARATHON data was used to produce results for the inclusive DIS cross section for these three gas targets. This measurement has provided the first extraction of the DIS cross section for ${ }^{3} \mathrm{H}$. Using the cross section measurements of the two $\mathrm{A}=3$ mirror nuclei and ${ }^{2} \mathrm{D}$, the MARATHON collaboration has calculated the $\mathrm{A}=3 \mathrm{EMC}$ effect for both ${ }^{3} \mathrm{He}$ and ${ }^{3} \mathrm{H}$. This experiment will provide the first ever results on the EMC effect of ${ }^{3} \mathrm{H}$ and the first on the comparison of the EMC effects of the two $\mathrm{A}=3$ mirror nuclei. The MARATHON EMC effect for ${ }^{3} \mathrm{He}$ agrees well with the previous JLab EMC measurement from experiment E03103 J. Seely and A. Daniels [79]. Due to this agreement, my analysis methods for the extraction of the EMC effect for ${ }^{3} \mathrm{He}$ are validated, and therefore are valid for the extraction the EMC effect for ${ }^{3} \mathrm{H}$. The measurement of both the ${ }^{3} \mathrm{He}$ and ${ }^{3} \mathrm{H}$ EMC effects are important because comparison of the EMC measurements between these two $\mathrm{A}=3$ nuclei can help evaluate 
isospin effects, and help remove model dependence of nuclear effects in the extraction of the $F_{2}^{n} / F_{n}^{p}$ structure function ratio.

The main goal of my analysis was to study the comparison of the EMC effect for the two $\mathrm{A}=3$ mirror nuclei. I show the comparison of the EMC effect for ${ }^{3} \mathrm{H}$ to the EMC effect for ${ }^{3} \mathrm{He}$ in figure 5.7. This comparison shows no difference from unity within the precision of the current status of the analysis.

The biggest sources of error for the EMC effect are the isoscalar correction and cross section model dependence. The MARATHON's measurement of the $F_{2}^{n} / F_{n}^{p}$ ratio will help improve the errors associated with the isoscalar correction once the MARATHON collaboration finishes the analysis. The $F_{2}^{n} / F_{n}^{p}$ produced by a ratio of ${ }^{3} \mathrm{H}$ and ${ }^{3} \mathrm{He}$ will greatly reduce the error of the structure function ratio at high values of $x$ due to the small differences in nuclear effects in comparing the two $\mathrm{A}=3$ mirror systems. A better understanding of the nucleon structure functions and the EMC effect will reduce the errors associated with the cross section extraction using a cross section model. Using an iterative procedure would help reduce the magnitude of the errors caused by the model dependence. A continuing goal of my analysis is to introduce an iterative procedure to correct the cross section model with data extracted cross sections.

The aim of this analysis is not to solve the EMC puzzle but to help find a part of the solution. The measurement of the EMC effect of ${ }^{3} \mathrm{H}$ will add to the pool of previously measured nuclei that will continue to grow. A proposed experiment at Jefferson Lab plans to expand the database of known EMC effects by measuring the EMC effect on a large range of light and heavy nuclei [42]. Another planned experiment at Jefferson Lab was proposed to provide new constraints on the EMC effect models by measuring the spin-dependent EMC effect on polarized ${ }^{7} \mathrm{Li}[27]$. Also, the flavor dependence of the EMC effect could be studied via pion induced Drell-Yan scattering[37] or tagged deep inelastic scattering measurements using the ALERT (Low Energy Recoil Tracker) detector in Hall B[6]. 


\section{Bibliography}


[1] Proceedings: International Symposium on Electron and Photon Interactions at High Energies, 3rd, Slac, 1967, 1967. x, 5

[2] A. Airapetian et al. Measurement of $\mathrm{R}=\operatorname{sigma}(\mathrm{L}) / \operatorname{sigma}(\mathrm{T})$ in deep inelastic scattering on nuclei. 2002. 20

[3] S. V. Akulinichev, S. Shlomo, S. A. Kulagin, and G. M. Vagradov. Lepton-nucleus deep-inelastic scattering. Phys. Rev. Lett., 55:2239-2241, Nov 1985. 23

[4] J. Alcorn et al. Basic Instrumentation for Hall A at Jefferson Lab. Nucl. Instrum. Meth., A522:294-346, 2004. xi, 27, 28, 29, 30, 32, 41, 42, 54, 57, 58, 79, 88

[5] P. Amaudruz et al. The ratio f2n/f2p in deep inelastic muon scattering. Nuclear Physics $B, 371(1): 3-31,1992.18$

[6] Whitney Armstrong et al. Tagged EMC Measurements on Light Nuclei. 2017. 96

[7] M. Arneodo et al. Measurement of the proton and the deuteron structure functions, F2(p) and F2(d). Phys. Lett., B364:107-115, 1995. 88

[8] J. Arrington, F. Coester, R. J. Holt, and T. S. H. Lee. Neutron Structure Functions. J. Phys., G36:025005, 2009. 91

[9] J. Arrington, R. Ent, C. E. Keppel, J. Mammei, and I. Niculescu. Low-q scaling, duality, and the emc effect. Phys. Rev. C, 73:035205, Mar 2006. x, 21, 22

[10] J. Ashman et al. Measurement of the ratios of deep inelastic muon-nucleus cross sections on various nuclei compared to deuterium. Physics Letters B, 202(4):603-610, 1988. 17

[11] J. J. Aubert et al. Measurement of the nucleon structure function f2 in muon-iron interactions at 120,250 and 280 gev. Physics Letters B, 105(4):322 - 328, 1981. 17

[12] J. J. Aubert et al. Measurement of the proton structure function $F_{2}$ in muon - hydrogen interactions at 120-GeV and 280-GeV. Phys. Lett., B105:315, 1981. x, 15, 16, 17

[13] J. J. Aubert et al. Measurement of the deuteron structure function f2d and a comparison of proton and neutron structure. Physics Letters B, 123(1):123 - 126, 1983. 17 
[14] J. J. Aubert et al. The ratio of the nucleon structure functions f2n for iron and deuterium. Physics Letters B, 123(3):275 - 278, 1983. 17

[15] T. Averett, B. Zhao, and B Wojtekhowski. Bigbite heavy gas cherenkov detector at 6.6 and 8.8 gev. xi, 45,46

[16] W B. Atwood, J D. Bjorken, Stanley Brodsky, and R Stroynowski. Lectures on lepton nucleon scattering and quantum chromodynamics. 01 1982. x, 9, 12

[17] J. Hague Bane, T. Kutz, H. Liu, M. Nycz, T. Su, and E. MClellan. Marathon pass 2 analysis primer. texnote, 2019. xi, 44, 45, 72

[18] G. Bari and other. A measurement of nuclear effects in deep inelastic muon scattering on deuterium, nitrogen and iron targets. Physics Letters B, 163(1):282 - 286, 1985. x, $17,18,22$

[19] W. Bartel et al. Electroproduction of pions near the (1236) isobar and the form factor gm (q2) of the (n)-vertex. Physics Letters B, 28(2):148 - 151, 1968. x, 7, 8

[20] O. Benhar, V.R. Pandharipande, and I. Sick. Nuclear binding and deep inelastic scattering. Physics Letters B, 410(2):79 - 85, 1997. 24

[21] R P Bickerstaff and A W Thomas. The EMC effect-with emphasis on conventional nuclear corrections. Journal of Physics G: Nuclear and Particle Physics, 15(10):15231569, oct 1989. 23, 24

[22] A. Bodek et al. Electron scattering from nuclear targets and quark distributions in nuclei. Phys. Rev. Lett., 50:1431-1434, May 1983. 88

[23] A. Bodek and U. K. Yang. Modeling deep inelastic cross-sections in the few GeV region. Nucl. Phys. Proc. Suppl., 112:70-76, 2002. [,70(2002)]. xiii, 76, 88, 90

[24] C. Bourrely and Jacques Soffer. Deep inelastic scattering of leptons and hadrons in the QCD parton model and experimental tests. pages 1565-1581, 2001. 13

[25] Gennady M. Briskin. Diffractive Dissociation in ep Deep Inelastic Scattering. PhD thesis, Tel Aviv U., 1998. 9 
[26] Stanley J. Brodsky, Ivan Schmidt, and Jian-Jun Yang. Nuclear antishadowing in neutrino deep inelastic scattering. Phys. Rev., D70:116003, 2004. 16

[27] W. K. Brooks and S. Kuhn. The emc effect in spin structure functions. 96

[28] C. G. Callan and David J. Gross. High-energy electroproduction and the constitution of the electric current. Phys. Rev. Lett., 22:156-159, Jan 1969. 11

[29] I. C. Cloet, W. Bentz, and A. William Thomas. EMC and polarized EMC effects in nuclei. Phys. Lett., B642:210-217, 2006. 24

[30] F.E. Close, R.G. Roberts, and G.G. Ross. The effect of confinement size on nuclear structure functions. Physics Letters B, 129(5):346 - 350, 1983. 24

[31] Hall A Collaboration. ESPACE Manual, Event Scanning Program for Hall A Collaboration Experiments. 54, 55

[32] JLab MARATHON Collaboration. Measurment of the $f_{2}^{n} / f_{2}^{p}$, d/u ratios and a -3 emc effect in deep inelatic electron scattering off the tritium and helium mirror nuclei. Technical report, Jefferson Lab, 2010. 25

[33] A. Daniel. Precise measurement of the nuclear dependence of the EMC effect at large $x$. PhD thesis, University of Houston, 2007. xiii, 14, 16, 17, 18, 20, 23, 24, 78

[34] S. Dasu. Precision measurement of $\mathrm{x}, \mathrm{q} 2$ and a-dependence of $\mathrm{rl} / \mathrm{rt}$ and $\mathrm{f} 2$ in deep inelastic scattering., 1988. 77, 78

[35] J. Denard and A. Saha. High accuracy beam current monitor system for cebaf's hall a. Chicago, 2001. Particle Accelerator Conference. xi, 37

[36] G. Dunne and A.W. Thomas. Deep inelastic scattering as a probe of nucleon and nuclear structure. Nuclear Physics A, 446(1):437 - 443, 1985. 23

[37] D. Dutta, J. C. Peng, I. C. Cloet, and D. Gaskell. Pion-induced Drell-Yan processes and the flavor-dependent EMC effect. Phys. Rev., C83:042201, 2011. 96 
[38] K. Nakamura et. al. Review of particle physics. Journal of Physics G: Nuclear and Particle Physics, 37(7A):075021, jul 2010. x, 10

[39] K.G. Fissum et al. Vertical drift chambers for the hall a high-resolution spectrometers at jefferson lab. Nuclear Instruments and Methods in Physics Research Section A: Accelerators, Spectrometers, Detectors and Associated Equipment, 474(2):108 - 131, 2001. xi, 42,43

[40] David J. Flay. Measurements of the Neutron Longitudinal Spin Asymmetry A1n and Flavor Decomposition in the Valence Quark Region. PhD thesis, Temple University, Philadelphia, PA, Aug 2014. x, 1, 3, 36, 37

[41] Jerome I. Friedman. Deep inelastic scattering: Comparisons with the quark model. Rev. Mod. Phys., 63:615-629, 1991. 11

[42] D. Gaskell, J. Arrington, A. Daniel, and N. Fomin. Detailed studies of the nuclear dependence of $\mathrm{f} 2$ in light nuclei. 96

[43] D.F. Geesaman, K Saito, and A Thomas. The nuclear emc effect. Annual Review of Nuclear and Particle Science, 45(1):337-390, 1995. 23, 24

[44] M. Gell-Mann. The eightfold way: A theory of strong interaction symmetry. 13

[45] J. Gomez et al. Measurement of the $a$ dependence of deep-inelastic electron scattering. Phys. Rev. D, 49:4348-4372, May 1994. x, 18, 19, 20, 22, 111

[46] S.A. Goudsmit. The discovery of the electron spin. 4

[47] Daniel Greenberger and Friedel Weinert. Compendium of Quantum Physics: Concepts, Experiments, History and Philosophy. Springer Publishing Company, Incorporated, 2016. 4,13

[48] Franz Gross and S. Liuti. Role of nuclear binding in the european-muon-collaboration effect. Phys. Rev. C, 45:1374-1381, Mar 1992. x, 22

[49] Tyler Hague. Calibrating the hall a raster. Technical report, JLab, 2019. xi, 35, 36 
[50] K M Hanna, M E Buzaladze, V R Garsevanishvili, V A Matveev, and Z R Menteshashvili. Multiquark cluster model at large bjorken scaling variable. Journal of Physics G: Nuclear and Particle Physics, 19(5):721-730, may 1993. 23

[51] Florian Hauenstein and Reynier Cruz Torres. Tritium trigger setup. xii, 49

[52] D. Higinbotham. The emc effect still puzzles after 30 years - cern courier. http: //cerncourier.com/cws/article/cern/53091, April 2013. (Visited on 01/04/2016). $\mathrm{x}, 2,13,14,15$

[53] Doug Higinbotham. Target images, December 2017. 40

[54] R Hofstadter. Nuclear and nucleon scattering of high-energy electrons. Annual Review of Nuclear Science, 7(1):231-316, 1957. 5

[55] R. L. Jaffe. Quark distributions in nuclei. Phys. Rev. Lett., 50:228-231, Jan 1983. 23

[56] Zhihong Ye John Arrington. Marathon rate estimations. Rate Estimation, 2015. viii, 52

[57] R. Kazimi et al. Four Beam Generation for Simultaneous Four-Hall Operation at CEBAF. In Proc. of International Particle Accelerator Conference (IPAC'16), Busan, Korea, May 8-13, 2016, number 7 in International Particle Accelerator Conference, pages 4240-4242, Geneva, Switzerland, June 2016. JACoW. doi:10.18429/JACoWIPAC2016-THPOY060. 27

[58] Henry W. Kendall. Deep inelastic scattering: Experiments on the proton and the observation of scaling. Rev. Mod. Phys., 63:597-614, Jul 1991. 9

[59] S. A. Kulagin and R. Petti. Structure functions for light nuclei. Phys. Rev. C, 82:054614, Nov 2010. 88, 91

[60] Tyler Kutz. Tritium decay. Private communication, 2018. xiii, 71

[61] Christoph W. Leemann, David R. Douglas, and Geoffrey A. Krafft. The continuous electron beam accelerator facility: Cebaf at the jefferson laboratory. Annual Review of Nuclear and Particle Science, 51(1):413-450, 2001. 27, 28 
[62] Nilanga Liyanage. Optics calibration of the hall a high reolution spectrometers using the new optimizer, 2002. xii, 54, 55, 56, 57

[63] Larry L Lucas and Michael P Unterweger. Comprehensive review and critical evaluation of the half-life of tritium. Journal of research of the National Institute of Standards and Technology, 105(4):541, 2000. 70

[64] Simona Malace, David Gaskell, Douglas W. Higinbotham, and Ian Cloet. The Challenge of the EMC Effect: existing data and future directions. Int. J. Mod. Phys., E23:1430013, 2014. 14

[65] R. W. McAllister and R. Hofstadter. Elastic scattering of 188-mev electrons from the proton and the alpha particle. Phys. Rev., 102:851-856, May 1956. 1

[66] David Meekings. Hall a tritium target, 2017. viii, 39, 73, 86, 107

[67] L. W. MO and Y. S. TSAI. Radiative corrections to elastic and inelastic ep and up scattering. Rev. Mod. Phys., 41:205-235, Jan 1969. 77, 78

[68] Jonathan Mulholland. SANE's Measurement of the Proton's Virtual Photon Spin Asymmetry, $A_{1}^{p}$, at Large Bjorken x. PhD thesis, Virginia U., 2012. x, 3, 5, 13, 27

[69] O. Nachtmann and H.J. Pirner. Emc. Z. Phys. C., 21:277, 1984. 24

[70] NMC. Detailed measurements of structure functions from nucleons and nuclei. Technical Report CERN-SPSC-85-18. SPSC-P-210, CERN, Geneva, 1985. 18

[71] The New Muon Collaboration (NMC). The ratio f2n/f2p in deep inelastic muon scattering. Nuclear Physics B, 371(1):3 - 31, 1992. 88, 91

[72] P R Norton. The emc effect. Reports on Progress in Physics, 66(8):1253, 2003. 14, 18, 23

[73] Mike Nyzc. EMC effect in Tritium. PhD thesis, Kent State University, 2019. xi, 38, 39 
[74] G. Palacios-Serrano, F. Hannon, C. Hernandez-Garcia, M. Poelker, and H. Baumgart. Electrostatic design and conditioning of a triple point junction shield for a $200 \mathrm{kv} \mathrm{dc}$ high voltage photogun. Review of Scientific Instruments, 89(10):104703, 2018. 27

[75] D. H. Perkins. Introduction to high energy physics, 3rd edition. Addison-Wesley Pub Co Inc, United States, 1986. x, 11

[76] B. Povh. Particles and nuclei: an introduction to the physical concepts. Springer, 1999. $\mathrm{x}, 1,2,3,4,5,6,7,8,9,11,12,13,16,72$

[77] Tom Powers, Lawrence Doolittle, Rok Ursic, and Jeffrey Wagner. Design, commissioning and operational results of wide dynamic range bpm switched electrode electronics. AIP Conference Proceedings, 390(1):257-265, 1997. 31

[78] S.N. Santiesteban et al. Density changes in low pressure gas targets for electron scattering experiments. Nuclear Instruments and Methods in Physics Research Section A: Accelerators, Spectrometers, Detectors and Associated Equipment, 940:351 - 358, 2019. viii, 73,74

[79] J. Seely, A. Daniel, et al. New measurements of the european muon collaboration effect in very light nuclei. Phys. Rev. Lett., 103:202301, Nov 2009. xi, xiii, 2, 21, 23, 90, 91, 95

[80] Jason Seely. Precise Measurement of the Nuclear Dependence of Strucuture Functions in Light Nucleii. PhD thesis, Massachusetts Institiute of Technology, 2006. 14, 16, 20, 21,78

[81] J. R. Smith and G. A. Miller. Chiral solitons in nuclei: Saturation, EMC effect and Drell-Yan experiments. Phys. Rev. Lett., 91:212301, 2003. [Erratum: Phys. Rev. Lett.98,099902(2007)]. 24

[82] Tong Su. Positron correction analysis., 2019. xiii, 70

[83] Hall A Tech. Ep method. http://hallaweb.jlab.org/equipment/beam/energy/ep_ web.html, 2016. (Accessed on 06/28/2016). 37 
[84] Stein Emil Vollset. Confidence intervals for a binomial proportion. Statistics in Medicine, 12(9):809-824, 1993.

[85] S Wexler. Dissociation of th and t2 by $\beta$-decay. Journal of Inorganic and Nuclear Chemistry, 10(1-2):8-16, 1959. 70

[86] Pengjia Zhu et al. Beam Position Reconstruction for the g2p Experiment in Hall A at Jefferson Lab. Nucl. Instrum. Meth., A808:1-10, 2016. xi, 31, 32, 33 
Appendices 


\section{A Cross Section Tables}

This appendix contains the cross section tables for the three gas targets, ${ }^{3} \mathrm{H},{ }^{3} \mathrm{He}$, and D. The data used to produce the cross section for these targets was acquired during the MARATHON experiment completed at JLab. The error for the three gas targets' thickness measurements are: ${ }^{3} \mathrm{H}=0.97 \%,{ }^{3} \mathrm{He}=1.12 \%$, and ${ }^{2} \mathrm{D}=0.56 \%$ [66]. The errors contained in the table are the relative contribution to the cross section. The cuts used to produce the tables:

\section{Electron Selection Cuts}

- Number of tracks $\quad==1$

- MARATHON trigger (bit) \& $(1<<2)$

- Total Cherenkov ADC sum > 1800

- Calo. Layer 1 Energy $>1 \mathrm{GeV}$

- Calo. Layer 2 Energy $>0.6 \mathrm{GeV}$

- $\mathrm{W}^{2}>2.5$

- $-0.07 \mathrm{~m} \quad>=$ Vertex $\mathrm{Z} \quad<=0.09 \mathrm{~m}$

- $-0.035 \quad>=\delta_{t g} \quad<=0.035$

- $-0.04 \mathrm{rad} \quad>=\theta_{t g} \quad<=0.04 \mathrm{rad}$

- $-0.025 \mathrm{rad} \quad>=\phi_{t g} \quad<=0.025 \mathrm{rad}$ 
Table A.1: Cross section table for ${ }^{3} \mathrm{H}$.

\begin{tabular}{|l|l|l|l|l|l|l|l|l|l|}
\hline $\mathrm{X}$ & Q2 & $\begin{array}{l}\text { Cross } \\
\text { Section }\end{array}$ & $\begin{array}{l}\text { Stat. } \\
\text { Error }\end{array}$ & $\begin{array}{l}\text { Density } \\
\text { Cor. }\end{array}$ & $\begin{array}{l}\text { Positron } \\
\text { Sub. }\end{array}$ & $\begin{array}{l}\text { Endcap } \\
\text { Sub. }\end{array}$ & $\begin{array}{l}\text { Detector } \\
\text { Eff. }\end{array}$ & $\begin{array}{l}\text { MC \& Model } \\
\text { Error }\end{array}$ & $\begin{array}{l}\text { Cross Section } \\
\text { Error }\end{array}$ \\
\hline 0.185 & 2.708 & 18.776 & 0.0055 & 0.002 & 0.0004 & 0.007 & 0.004 & 0.016 & 0.019 \\
\hline 0.215 & 3.055 & 15.49 & 0.0045 & 0.002 & 0.0002 & 0.007 & 0.004 & 0.014 & 0.017 \\
\hline 0.245 & 3.446 & 11.996 & 0.0049 & 0.002 & 0.0002 & 0.007 & 0.0041 & 0.013 & 0.016 \\
\hline 0.275 & 3.881 & 8.617 & 0.0051 & 0.002 & 0.0001 & 0.007 & 0.0045 & 0.014 & 0.017 \\
\hline 0.305 & 4.311 & 6.28 & 0.0059 & 0.002 & 0.0002 & 0.007 & 0.0051 & 0.014 & 0.018 \\
\hline 0.335 & 4.705 & 4.931 & 0.0073 & 0.002 & 0.0002 & 0.007 & 0.0057 & 0.014 & 0.018 \\
\hline 0.365 & 5.122 & 3.767 & 0.0082 & 0.002 & 0.0002 & 0.007 & 0.0067 & 0.014 & 0.019 \\
\hline 0.395 & 5.55 & 2.794 & 0.0098 & 0.002 & 0.0002 & 0.007 & 0.0079 & 0.013 & 0.02 \\
\hline 0.425 & 6.023 & 2.049 & 0.0091 & 0.002 & 0.0001 & 0.007 & 0.0091 & 0.013 & 0.02 \\
\hline 0.455 & 6.397 & 1.591 & 0.009 & 0.002 & 0.0001 & 0.007 & 0.0091 & 0.013 & 0.02 \\
\hline 0.49 & 6.922 & 1.153 & 0.01 & 0.002 & 0.0001 & 0.007 & 0.0083 & 0.013 & 0.02 \\
\hline 0.53 & 7.472 & 0.776 & 0.0086 & 0.002 & 0.0001 & 0.007 & 0.0075 & 0.014 & 0.019 \\
\hline 0.57 & 8.045 & 0.527 & 0.0111 & 0.002 & 0.0001 & 0.007 & 0.0071 & 0.016 & 0.022 \\
\hline 0.61 & 8.617 & 0.351 & 0.0102 & 0.002 & 0.0001 & 0.007 & 0.0064 & 0.02 & 0.024 \\
\hline 0.655 & 9.241 & 0.22 & 0.0115 & 0.002 & 0.0001 & 0.007 & 0.0055 & 0.025 & 0.029 \\
\hline 0.705 & 9.997 & 0.121 & 0.0113 & 0.002 & 0.0 & 0.007 & 0.0041 & 0.03 & \\
\hline 0.76 & 10.688 & 0.065 & 0.0109 & 0.002 & 0.0 & 0.007 & 0.0035 & 0.026 & 0.033 \\
\hline 0.825 & 11.468 & 0.03 & 0.0143 & 0.002 & 0.0 & 0.007 & 0.0032 & 0.037 & 0.029 \\
\hline
\end{tabular}


Table A.2: Cross section table for ${ }^{3} \mathrm{H}$.

\begin{tabular}{|l|l|l|l|l|l|l|l|l|l|}
\hline $\mathrm{X}$ & Q2 & $\begin{array}{l}\text { Cross } \\
\text { Section }\end{array}$ & $\begin{array}{l}\text { Stat. } \\
\text { Error }\end{array}$ & $\begin{array}{l}\text { Density } \\
\text { Cor. }\end{array}$ & $\begin{array}{l}\text { Positron } \\
\text { Sub. }\end{array}$ & $\begin{array}{l}\text { Endcap } \\
\text { Sub. }\end{array}$ & $\begin{array}{l}\text { Detector } \\
\text { Eff. }\end{array}$ & $\begin{array}{l}\text { MC \& Model } \\
\text { Error }\end{array}$ & $\begin{array}{l}\text { Cross Section } \\
\text { Error }\end{array}$ \\
\hline 0.185 & 2.709 & 20.71 & 0.0063 & 0.002 & 0.0006 & 0.007 & 0.004 & 0.016 & 0.019 \\
\hline 0.215 & 3.059 & 17.303 & 0.0051 & 0.002 & 0.0004 & 0.007 & 0.004 & 0.015 & 0.018 \\
\hline 0.245 & 3.447 & 13.59 & 0.0052 & 0.002 & 0.0002 & 0.007 & 0.0041 & 0.016 & 0.019 \\
\hline 0.275 & 3.88 & 9.842 & 0.0056 & 0.002 & 0.0002 & 0.007 & 0.0044 & 0.017 & 0.02 \\
\hline 0.305 & 4.313 & 7.22 & 0.0064 & 0.002 & 0.0002 & 0.007 & 0.0051 & 0.017 & 0.02 \\
\hline 0.335 & 4.708 & 5.803 & 0.0074 & 0.002 & 0.0002 & 0.007 & 0.0058 & 0.017 & 0.021 \\
\hline 0.365 & 5.122 & 4.446 & 0.0081 & 0.002 & 0.0002 & 0.007 & 0.0067 & 0.016 & 0.02 \\
\hline 0.395 & 5.549 & 3.419 & 0.0095 & 0.002 & 0.0002 & 0.007 & 0.0079 & 0.015 & 0.021 \\
\hline 0.425 & 6.024 & 2.499 & 0.009 & 0.002 & 0.0002 & 0.007 & 0.0091 & 0.014 & 0.02 \\
\hline 0.455 & 6.397 & 1.974 & 0.0089 & 0.002 & 0.0002 & 0.007 & 0.0091 & 0.013 & 0.02 \\
\hline 0.49 & 6.924 & 1.434 & 0.0098 & 0.002 & 0.0001 & 0.007 & 0.0083 & 0.012 & 0.019 \\
\hline 0.53 & 7.473 & 0.985 & 0.0083 & 0.002 & 0.0001 & 0.007 & 0.0075 & 0.011 & 0.017 \\
\hline 0.57 & 8.044 & 0.668 & 0.0108 & 0.002 & 0.0001 & 0.007 & 0.0071 & 0.013 & 0.02 \\
\hline 0.61 & 8.616 & 0.447 & 0.0099 & 0.002 & 0.0001 & 0.007 & 0.0064 & 0.017 & 0.022 \\
\hline 0.655 & 9.242 & 0.282 & 0.0112 & 0.002 & 0.0001 & 0.007 & 0.0055 & 0.024 & 0.028 \\
\hline 0.705 & 9.998 & 0.158 & 0.0111 & 0.002 & 0.0001 & 0.007 & 0.0041 & 0.033 & 0.036 \\
\hline 0.76 & 10.689 & 0.087 & 0.0105 & 0.002 & 0.0 & 0.007 & 0.0035 & 0.035 & 0.037 \\
\hline 0.825 & 11.467 & 0.038 & 0.0141 & 0.002 & 0.0 & 0.007 & 0.0031 & 0.024 & 0.029 \\
\hline
\end{tabular}


Table A.3: Cross section table for D.

\begin{tabular}{|l|l|l|l|l|l|l|l|l|l|}
\hline $\mathrm{x}$ & $\mathrm{Q} 2$ & $\begin{array}{l}\text { Cross } \\
\text { Section }\end{array}$ & $\begin{array}{l}\text { Stat. } \\
\text { Error }\end{array}$ & $\begin{array}{l}\text { Density } \\
\text { Cor. }\end{array}$ & $\begin{array}{l}\text { Positron } \\
\text { Sub. }\end{array}$ & $\begin{array}{l}\text { Endcap } \\
\text { Sub. }\end{array}$ & $\begin{array}{l}\text { Detector } \\
\text { Eff. }\end{array}$ & $\begin{array}{l}\text { MC \& Model } \\
\text { Error }\end{array}$ & $\begin{array}{l}\text { Cross Section } \\
\text { Error }\end{array}$ \\
\hline 0.185 & 2.708 & 13.048 & 0.0062 & 0.002 & 0.0004 & 0.007 & 0.004 & 0.015 & 0.018 \\
\hline 0.215 & 3.061 & 10.858 & 0.0051 & 0.002 & 0.0002 & 0.007 & 0.004 & 0.013 & 0.016 \\
\hline 0.245 & 3.452 & 8.473 & 0.0051 & 0.002 & 0.0002 & 0.007 & 0.0041 & 0.013 & 0.016 \\
\hline 0.275 & 3.883 & 6.147 & 0.0049 & 0.002 & 0.0001 & 0.007 & 0.0045 & 0.014 & 0.017 \\
\hline 0.305 & 4.31 & 4.596 & 0.0053 & 0.0024 & 0.0002 & 0.007 & 0.0051 & 0.015 & 0.018 \\
\hline 0.335 & 4.695 & 3.724 & 0.0066 & 0.0027 & 0.0002 & 0.007 & 0.0055 & 0.015 & 0.019 \\
\hline 0.365 & 5.119 & 2.812 & 0.0083 & 0.0033 & 0.0001 & 0.007 & 0.0064 & 0.015 & 0.02 \\
\hline 0.395 & 5.549 & 2.111 & 0.0119 & 0.004 & 0.0001 & 0.007 & 0.0079 & 0.014 & 0.022 \\
\hline 0.425 & 6.023 & 1.574 & 0.0115 & 0.003 & 0.0001 & 0.007 & 0.0091 & 0.014 & 0.022 \\
\hline 0.455 & 6.397 & 1.279 & 0.0112 & 0.003 & 0.0001 & 0.007 & 0.0091 & 0.013 & 0.021 \\
\hline 0.49 & 6.944 & 0.868 & 0.0113 & 0.003 & 0.0001 & 0.007 & 0.0081 & 0.013 & 0.021 \\
\hline 0.53 & 7.474 & 0.615 & 0.0083 & 0.003 & 0.0001 & 0.007 & 0.0075 & 0.012 & 0.018 \\
\hline 0.57 & 8.045 & 0.427 & 0.0106 & 0.003 & 0.0001 & 0.007 & 0.0071 & 0.014 & 0.02 \\
\hline 0.61 & 8.615 & 0.286 & 0.0096 & 0.003 & 0.0 & 0.007 & 0.0064 & 0.017 & 0.022 \\
\hline 0.655 & 9.239 & 0.179 & 0.011 & 0.003 & 0.0 & 0.007 & 0.0055 & 0.022 & 0.026 \\
\hline 0.705 & 9.992 & 0.098 & 0.011 & 0.0026 & 0.0 & 0.007 & 0.0041 & 0.028 & 0.031 \\
\hline 0.76 & 10.688 & 0.052 & 0.0109 & 0.0022 & 0.0 & 0.007 & 0.0035 & 0.027 & 0.03 \\
\hline 0.825 & 11.467 & 0.023 & 0.0143 & 0.002 & 0.0 & 0.007 & 0.0032 & 0.026 & 0.031 \\
\hline
\end{tabular}




\section{B EMC Simulation}

Nuclei are systems of nucleons that interact strongly. The characteristic scale for the nucleons momentum is approximately the Fermi momentum, $k_{F} \approx 200-270 \mathrm{MeV} / \mathrm{c}$ [45]. However because of the strongly repulsive nature of the nucleon-nucleon interaction at short distances prevents two nucleons from laying in close proximity to each other. This strong interaction demands the presence of high-momentum components in the nuclear ground state wave function. A simulation was designed to phenomenologically study the effect of these high-momentum components on the nuclear EMC effect. This program was designed in two phases. The first phase used simple elastic scattering and a single value for the targets momentum to investigate overall effect of different target momentum on the yield in bins of $x_{B}$. The second phase of the simulation was created to lay out the effect of using different momentum distributions on the yield for the EMC effect region of $x_{B}, 0.3$ to 0.7.

\section{B.1 Investigation}

This simulation phenomenologically investigates the effect of a moving target on the EMC effect by scattering a beam of electrons off of a moving proton. The target protons are comprised of a directional vector of $0^{\circ}$ to $360^{\circ}$ in respect to the incoming electron beam and a momentum between 0 and $1 \mathrm{GeV} / \mathrm{c}$. Figure B.1 contains a possible event for the simulation. The electron approaches with $2.5 \mathrm{GeV}$ of energy and collides with a proton moving with a momentum of $0.5 \mathrm{GeV} / \mathrm{c}$ with an angle of $45^{\circ}$ in respect to the electron trajectory.

Using conservation of momentum and conservation of energy in elastic collisions, this simulation calculates the final state of the electron and proton after the scattering event by randomly selecting a scattered direction for the electron. The vector representation of the scattered products are shown in figure B.3a. In order to make these calculations systematic and to study cross sections models the simulation transform each event into the rest frame of the target before scattering. B.3a 


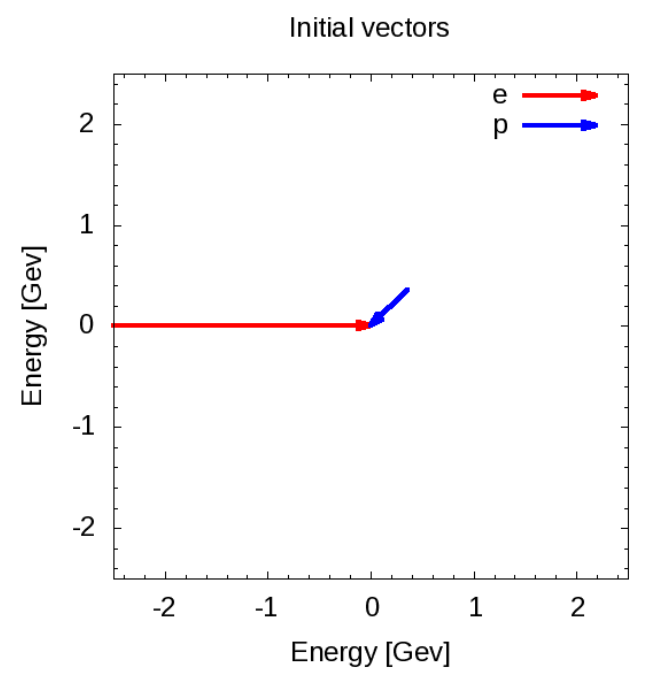

Figure B.1: Example of the electron beam (red) with an energy of $2.5 \mathrm{GeV}$ and the proton (blue) with angle of $45^{\circ}$ in respect to the electron and with a momentum of $0.5 \mathrm{GeV} / \mathrm{c}$.

\section{B.2 Transformation}

The Simulation completes a set of Lorentz invariant rotations and boost for each event to transform the lab frame of the electron and proton collision into the rest frame of the proton. First the simulation takes the initial proton and electron vectors and rotates them to align the proton vector to the horizontal axis, shown in figure B.2b.This rotation uses the angle between the proton and the electron defined as $\lambda$. This allows for a straight forward calculations for the Lorentz factors $\beta$ and $\gamma$ and to boost into the rest frame of the target proton, figure B.2c. Once in the boosted frame, the angle between the electron and the horizontal axis is defined as $\delta$. Right before the simulation starts to calculate the scattered products, it completes one more rotation to align the electron vector with the horizontal axis, figure B.2d, to make the scattering calculation systematic and unconditional.

In order to gain a more complete understanding of the scattering products, the program completes a set of transformations to move from the rest frame of the target proton to the beginning lab frame. After the simulation calculates the scattered products it begins to transform back by beginning with a rotation by the angle $\delta$, figure B.3b. Followed by the inverse of the previously used Lorentz boost. The last transformation, a rotation by $\lambda$, 


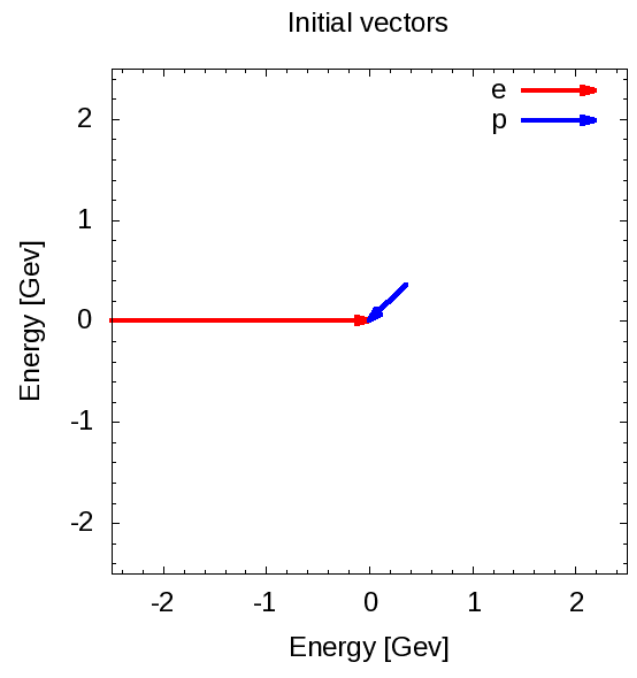

(a) Initial Vectors

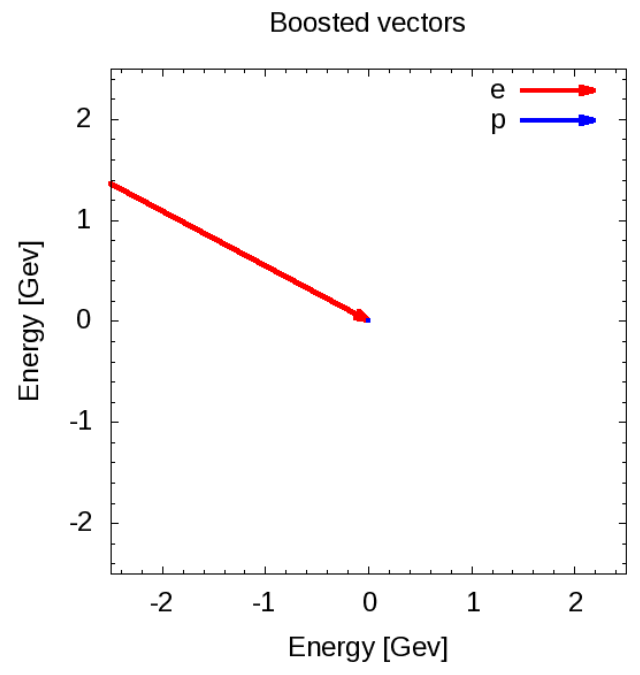

(c) Boosted

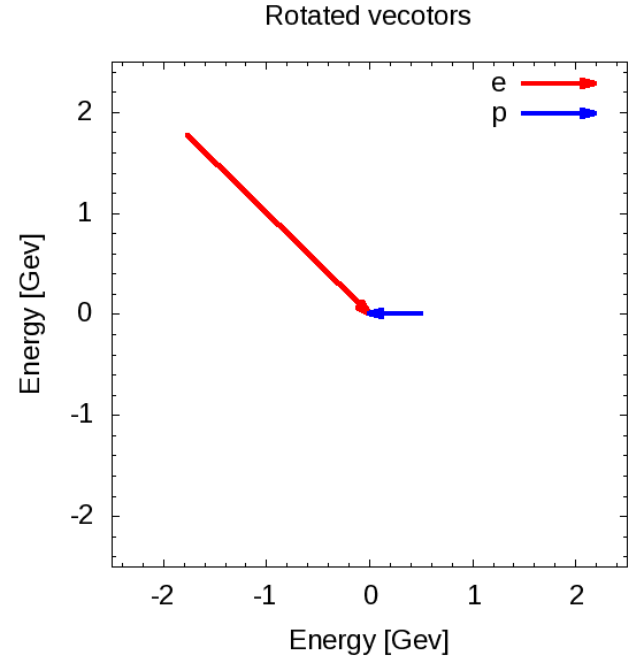

(b) Rotated by Lambda

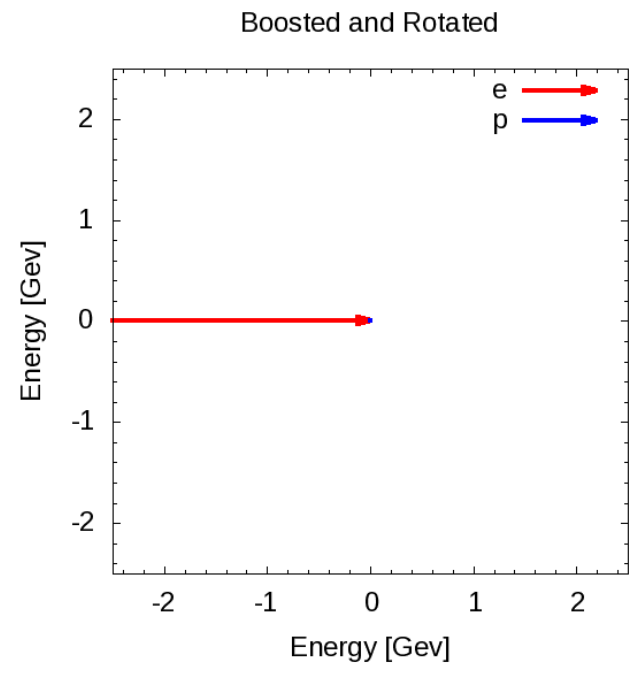

(d) Before being scattered

Figure B.2: Vector representations of the momentum for the incoming electron (red) and target proton (blue) with units of $\mathrm{GeV}$ for each phase of their transformations before scattering. 


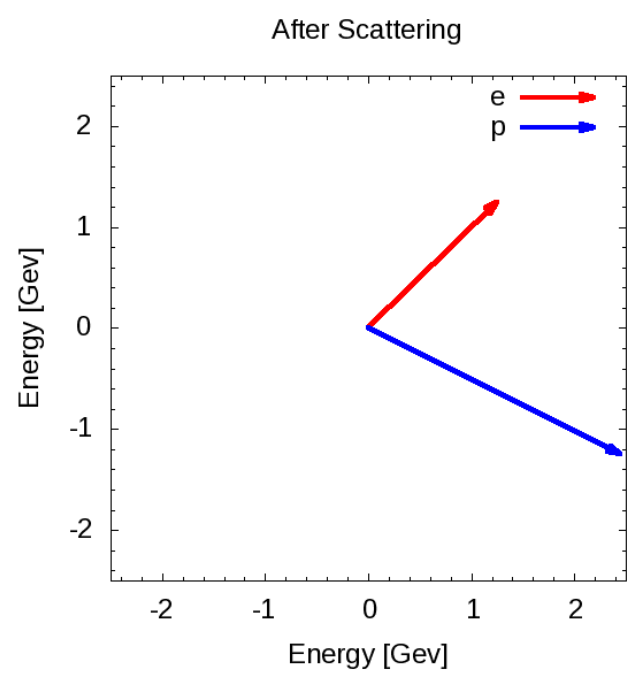

(a) After scattering

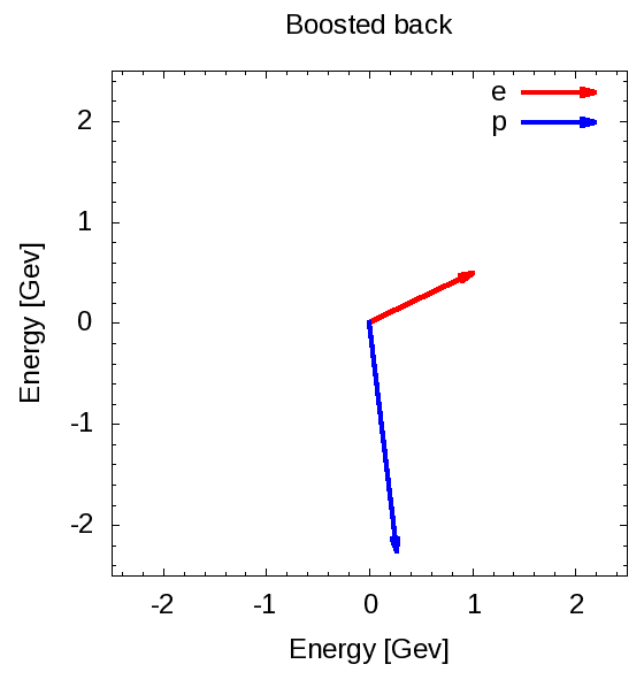

(c) Boosted

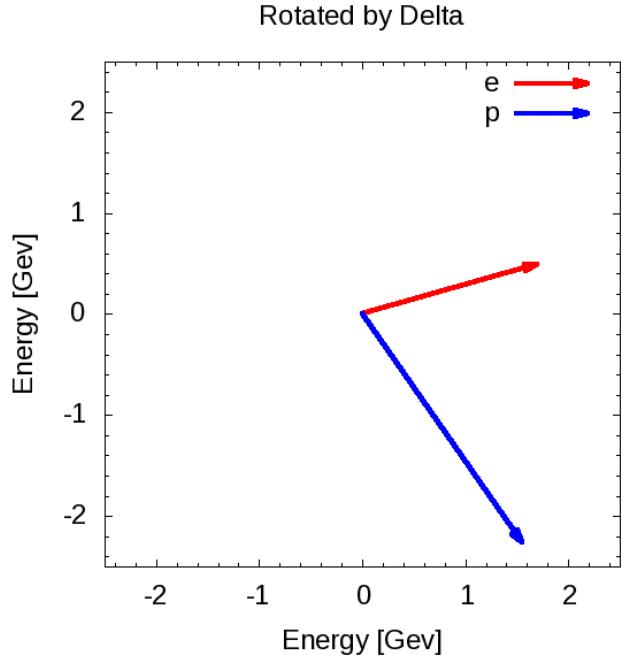

(b) Rotate back by $\delta$

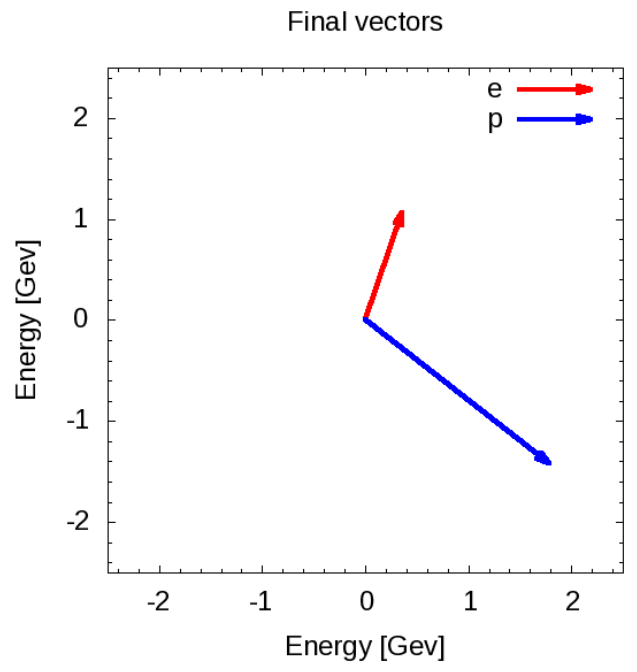

(d) Final vectors

Figure B.3: Vector representations of the momentum for the incoming electron (red) and target proton (blue) with units of $\mathrm{GeV}$ for each phase of their transformations after scattering). 


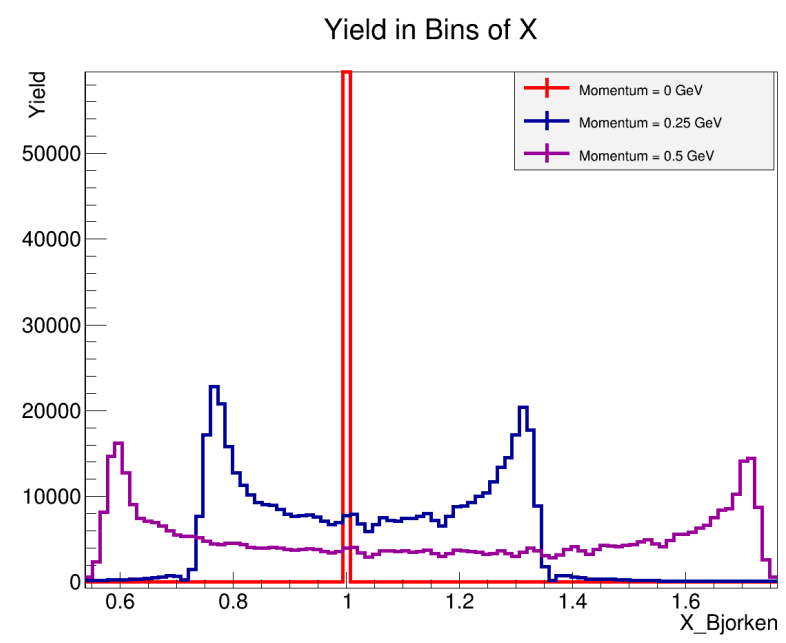

Figure B.4: Simulation results for fixed momentum protons. Three runs with unique proton momentum.

transforms the frame back into the lab frame. A proton vector and electron vector in the lab frame are the final products of the simulation. An image of the electron and proton vectors for each transformation can be found in figure B.3. These vectors allow for calculation of kinematic variables such as Bjoken $x$ and the four-momentum transfer $\left(Q^{2}\right)$. This simulation will complete these steps for many electron and proton combinations.

\section{B.3 Results}

This electron scattering simulation produced results for two stages. The firsts stage used a fix proton momentum for each run to compare the yield in bins of $x_{B}$. Figure B.4 shows the results for three different runs, each having a unique fixed proton momentum. The red histogram represents a run with a proton momentum of $0 \mathrm{Gev} / \mathrm{c}$. The result is an elastic peak at $x_{B}$ of one. The blue histogram contains the results having a fixed proton momentum of $0.25 \mathrm{GeV} / \mathrm{c}$. Increasing the initial momentum of the proton spreads the events into two peaks. The scattering interactions that form the peak above $1 x_{B}$ are produced by events were the proton's initial directional vector are orientated towards the electron. The events that produce an $x_{B}$ below 1 have a proton direction pointing away from the electron initially. Doubling the proton's initial momentum from $0.25 \mathrm{GeV}$ to $0.50 \mathrm{GeV}$ causes these peaks two spread out further in $x_{B}$. 


\section{Vita}

Jason Bane was born in Tennessee in 1985. Jason earned a B.S. in physics with a minor in secondary education focused on teaching science from the University of Tennessee. After his B.S., Jason continued school at UT to complete requirements for a secondary education teaching license. Using the teaching license that Jason acquired, he went to teach at Clay County High School in Celina, TN. Here in Celina, Jason taught physics, geometry, and senior level remedial math, along with coaching football both for the high school and the middle school.

Moving on from teaching, Jason returned to the University of Tennessee for a Ph.D. in physics. For Jason's first three years, he was a teaching assistant for both intro. physics and astronomy courses. After completing a stint of head Astronomy TA, Jason joined Prof. Fomin's group working at Thomas Jefferson National Accelerator Facility(JLab). During Jason's time at JLab, he has earned two separate fellowships, the DOE-Science Graduate Student Research (SCGSR) fellowship and Jefferson Science Associates(JSA) JLab Graduate Fellowship. Jason received chancellors honors for extraordinary professional promise during his first year at JLab. Also during his time at JLab, Jason has been a part of 5 peer review articles and participated in 7 national conference presentations and one international conference presentation. 